University of Louisville

ThinkIR: The University of Louisville's Institutional Repository

Electronic Theses and Dissertations

$12-2020$

\title{
College was a slap in the face: examining the transition of Kentucky Appalachian students studying STEM at an urban institution.
}

Heidi Neal

University of Louisville

Follow this and additional works at: https://ir.library.louisville.edu/etd

Part of the Student Counseling and Personnel Services Commons

\section{Recommended Citation}

Neal, Heidi, "College was a slap in the face: examining the transition of Kentucky Appalachian students studying STEM at an urban institution." (2020). Electronic Theses and Dissertations. Paper 3499.

https://doi.org/10.18297/etd/3499

This Doctoral Dissertation is brought to you for free and open access by ThinkIR: The University of Louisville's Institutional Repository. It has been accepted for inclusion in Electronic Theses and Dissertations by an authorized administrator of ThinkIR: The University of Louisville's Institutional Repository. This title appears here courtesy of the author, who has retained all other copyrights. For more information, please contact thinkir@louisville.edu. 
COLLEGE WAS A SLAP IN THE FACE: EXAMINING THE TRANSITION OF KENTUCKY APPALACHIAN STUDENTS STUDYING STEM AT AN URBAN INSTITUTTION

\author{
By \\ Heidi Neal \\ BS, Morehead State University, 2005 \\ MS, East Tennessee State University, 2007
}

\begin{abstract}
A Dissertation
Submitted to the Faculty of the College of Education and Human Development of the University of Louisville in Partial Fulfillment of the Requirements for the Degree of
\end{abstract}

Doctor of Philosophy in Educational Leadership and Organizational Leadership

Department of Education, Leadership, and Human Resource Development University of Louisville

Louisville, Kentucky

August 2020 

COLLEGE WAS A SLAP IN THE FACE: EXAMING THE TRANSITION OF KENTUCKY APPALACHIAN STUDENTS STUDYING STEM AT AN URBAN INSTITUTTION

\author{
By \\ Heidi Neal \\ BS, Morehead State University, 2005 \\ MS, East Tennessee State University, 2007 \\ Dissertation Approved on
}

July 7, 2020

By the following Dissertation Committee:

Dr. Casey George, Dissertation Director

Dr. Meera Alagaraja, Committee Member

Dr. Kerry Charron, Committee Member

Dr. Erin Gerber, Committee Member 


\section{ACKNOWLEDGMENTS}

This dissertation cannot be completed without the help and support of many individuals, I'd like to take the time to thank those who have made this accomplishment possible. First, I'd like to thank the participants. I'm thankful for their willingness to be

a part of the study and openly share about your experience. I would also like to thank my committee members, Dr. Meera Alagaraja, Dr. Kerry Charron, Dr. Erin Gerber, and especially my faculty advisor Dr. Casey George. You all have not only been my guiding light through this process, you pushed me to produce the best final piece possible. I'd like to thank Dr. Gail DePuy for providing unquestioned support and encouragement from the beginning of this journey to the finish line, I'm thankful for your mentorship and friendship. I'm thankful for my grandmother who was always the first person to say I was capable of completing such a difficult journey, she'd be awfully proud of this piece. I'm thankful for the support and love of my parents; you kept me focused on this goal and never let me see anything but the finish line. Most meaningfully, you always believed in me and my ability to accomplish anything I set my mind to; you helped me believe in myself. I'm thankful for the time I was able to spend writing this piece within the hills of Appalachia, a place that has always felt like home. I'm thankful for the support and love provided by you Superman, you were always there when I needed you. But most importantly, I would like to thank my children Abigail and Jack, I love you both with my entire heart. 


\begin{abstract}
COLLEGE WAS A SLAP IN THE FACE: EXAMING THE TRANSITION OF KENTUCKY APPALACHIAN STUDENTS STUDYING STEM AT AN URBAN INSTITUTTION
\end{abstract}

\author{
Heidi Neal
}

July 7, 2020

This phenomenological qualitative study examines the lived experience of fourteen rural Appalachian Kentucky (AKY) students studying STEM at an urban university. This study seeks to investigate the unique experiences of AKY students transitioning to STEM majors in college and examines the common factors between their transitional experience into college while addressing compounding factors of a rigorous STEM major and a new cultural setting at an urban institution. This study utilizes Schlossberg's transitional theory as the framework for analysis. Four key themes emerged from this data: a need for a strong support system, academic preparation, university focus on support and interventions, as well as individual adaptation. Key findings include a strong emphasis for support from both family and university is needed to enhance the adaptation throughout the transitional experience. 
TABLE OF CONTENTS

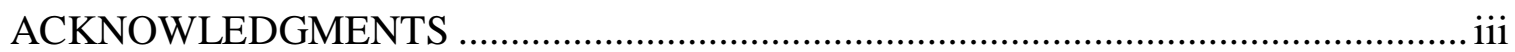

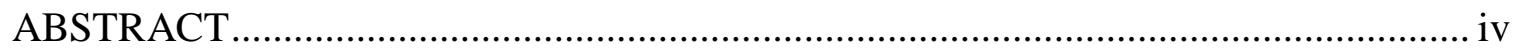

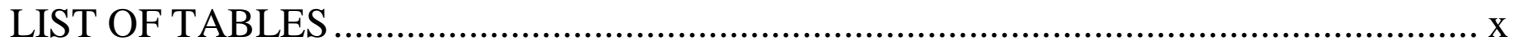

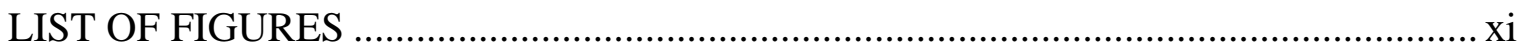

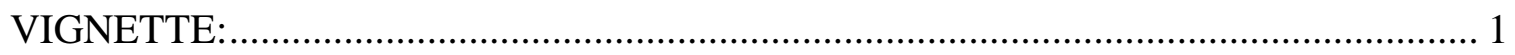

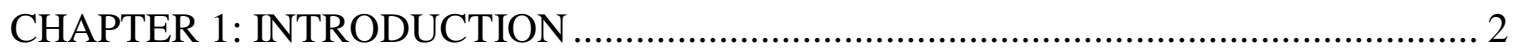

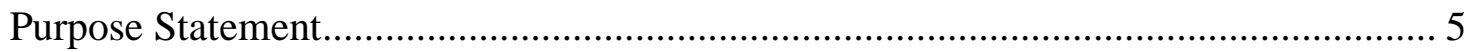

Research Question .............................................................................................. 5

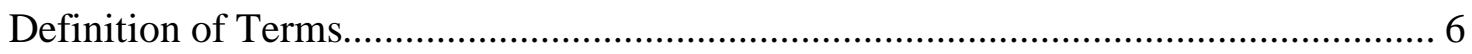

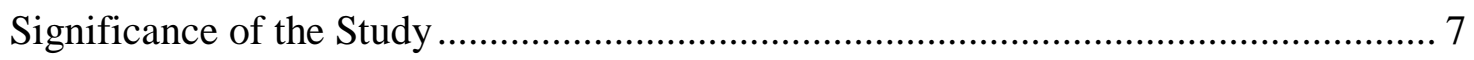

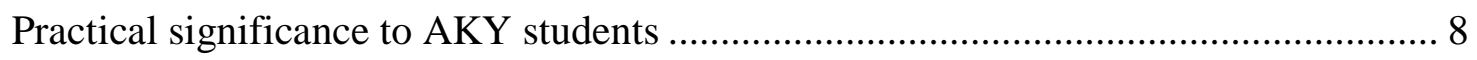

Practical significance to universities.................................................................... 8

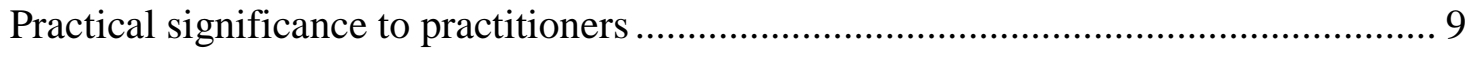

Practical significance to policymakers........................................................................ 9

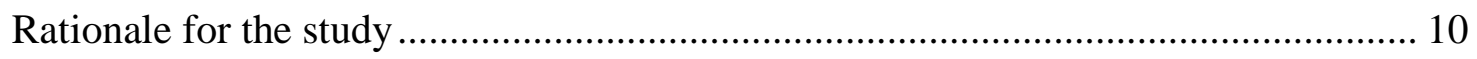

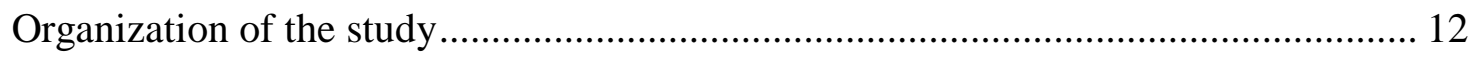

CHAPTER 2: LITERATURE REVIEW …………………................................... 13

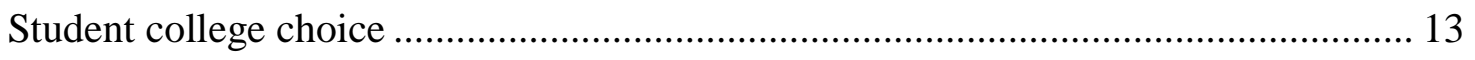




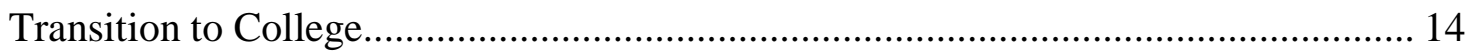

Student academic and social transition ................................................................... 16

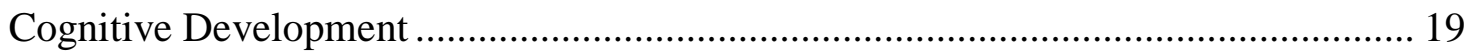

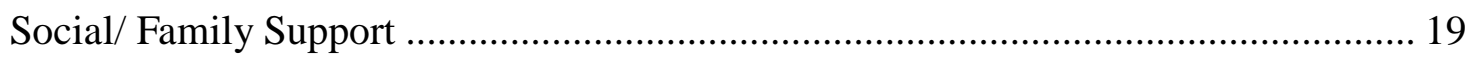

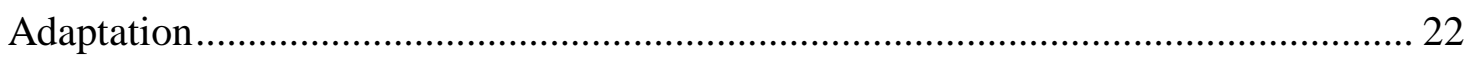

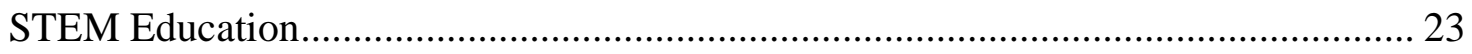

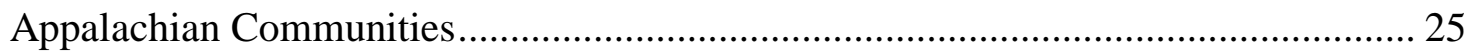

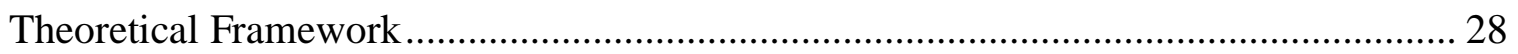

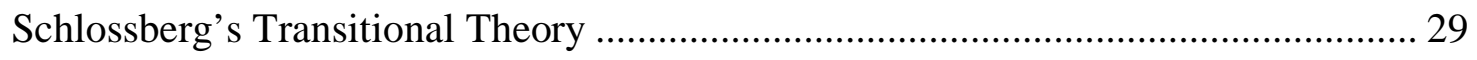

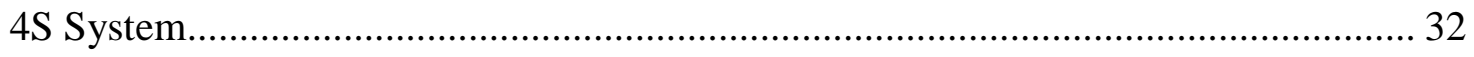

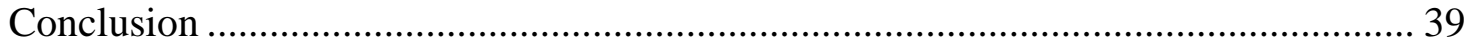

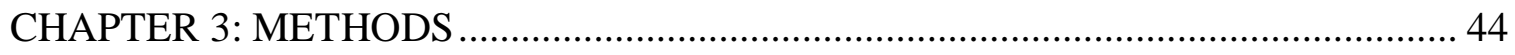

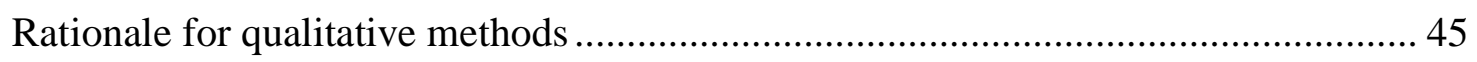

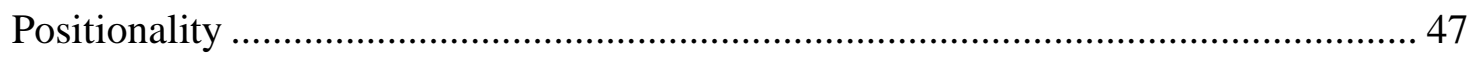

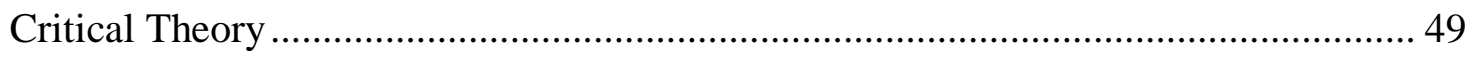

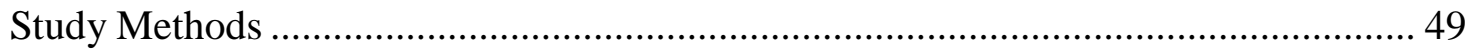

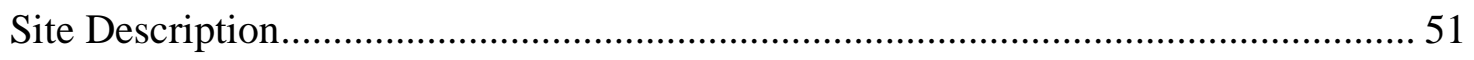

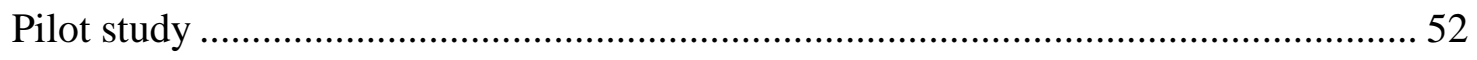

Participant 1 (Mary) .............................................................................................. 54

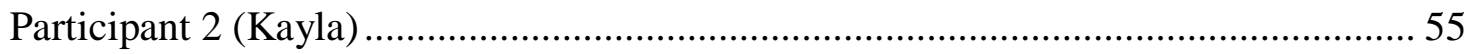




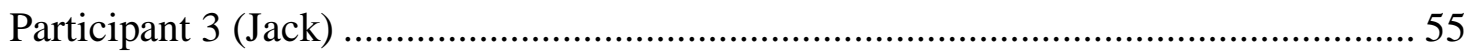

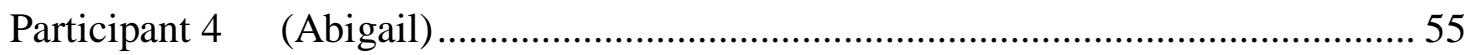

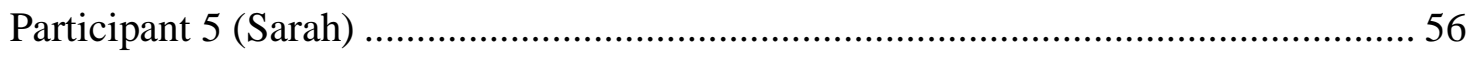

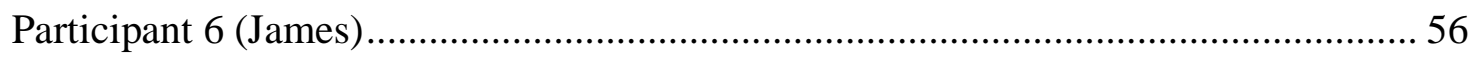

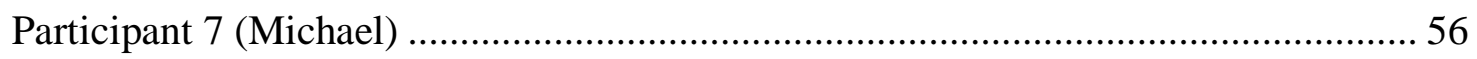

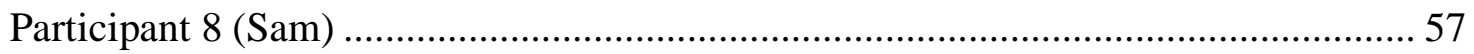

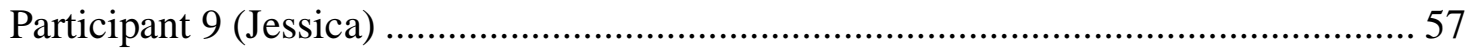

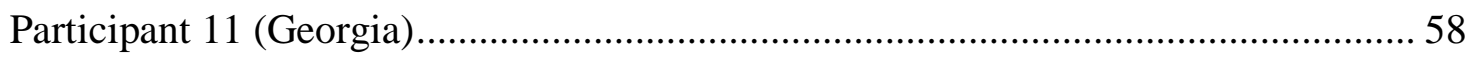

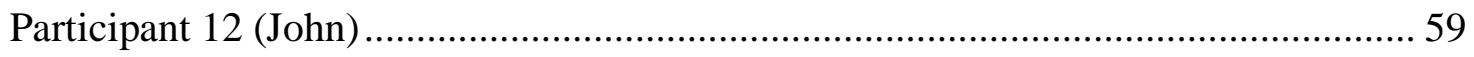

Participant 13 (Denise) ....................................................................................... 59

Participant 14 (Taylor) ........................................................................................ 59

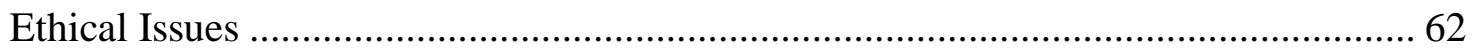

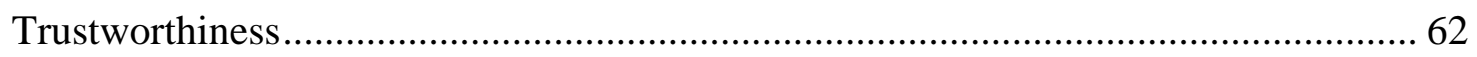

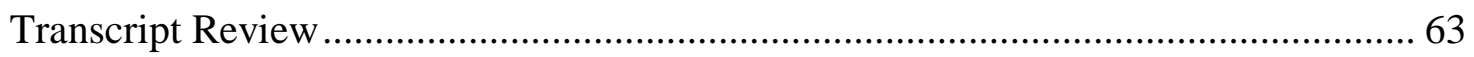

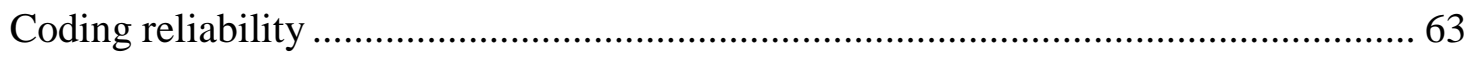

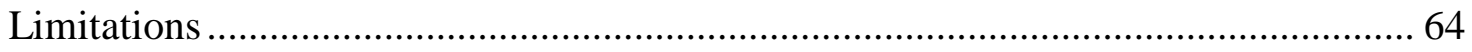

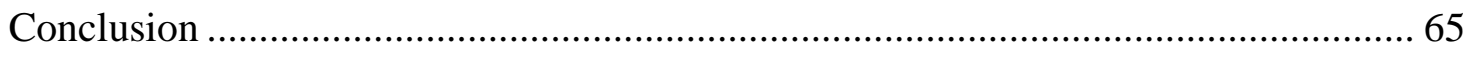

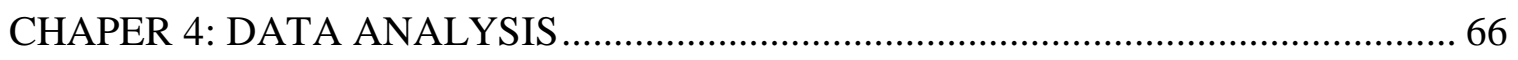

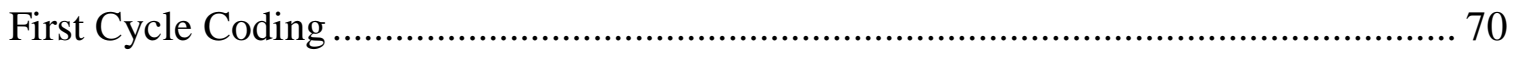

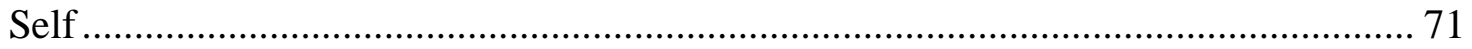




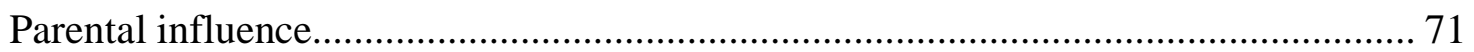

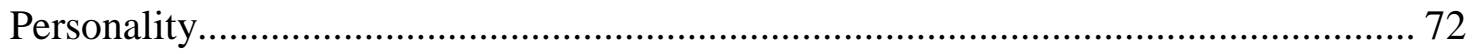

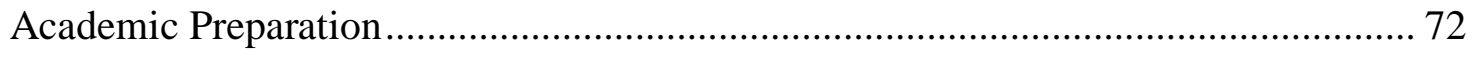

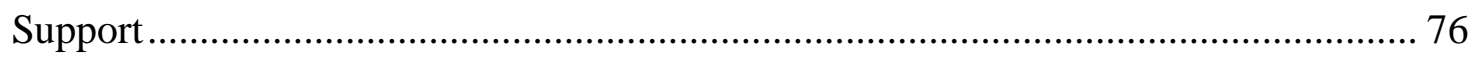

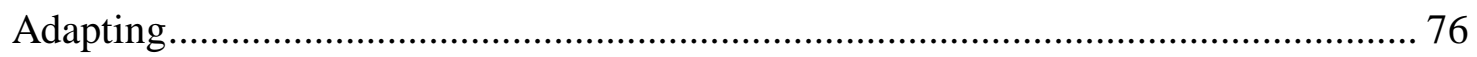

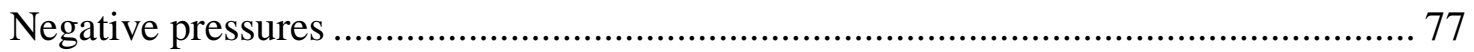

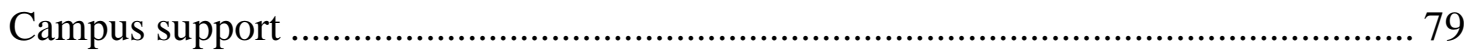

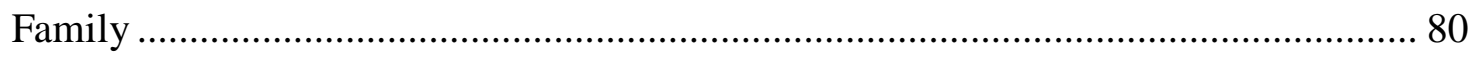

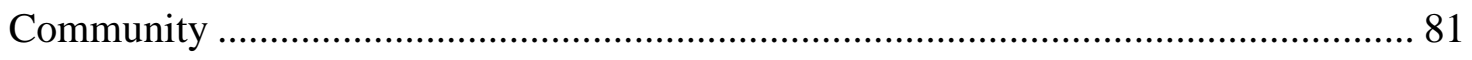

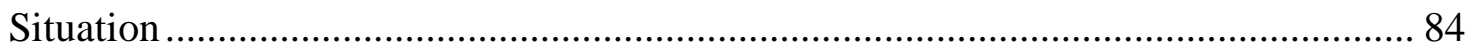

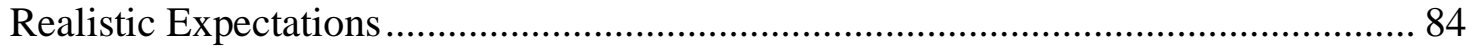

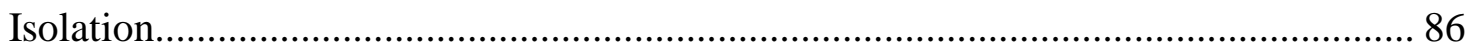

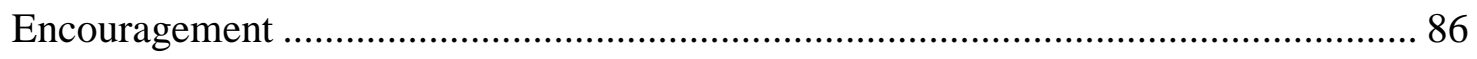

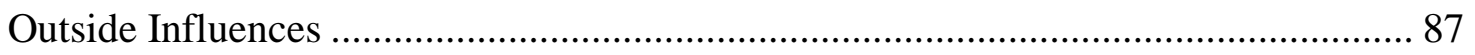

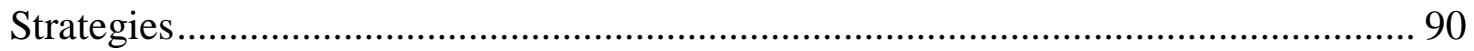

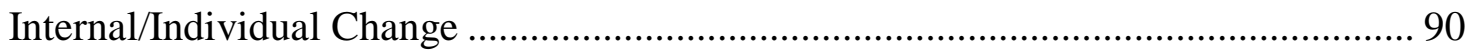

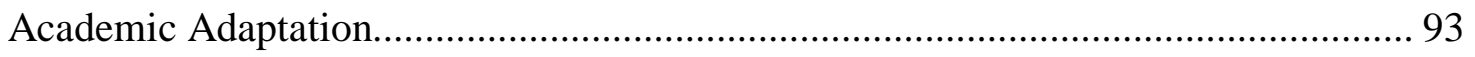

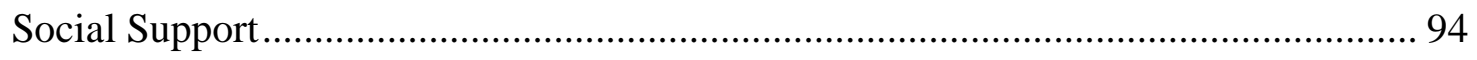

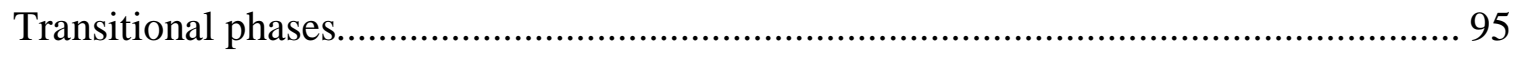

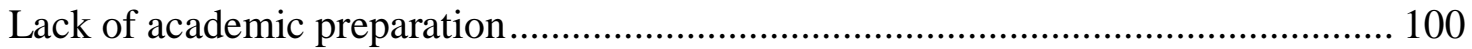


University support within social support/ assisting an individual theme

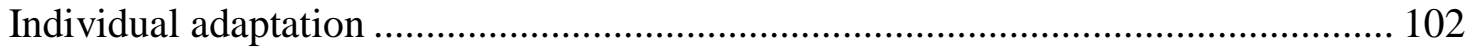

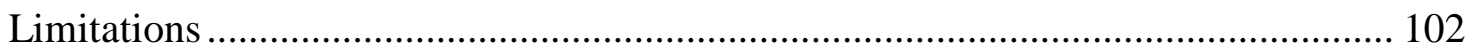

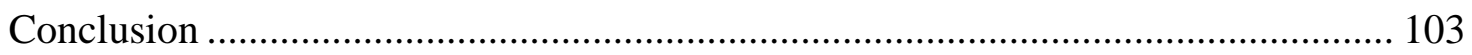

CHAPTHER V: CONCLUSIONS/ IMPLICATIONS ........................................... 105

Future Research Opportunities ................................................................... 105

Expansion of Schlossberg's transitional theory into college .................................. 105

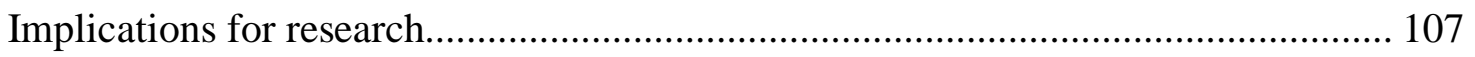

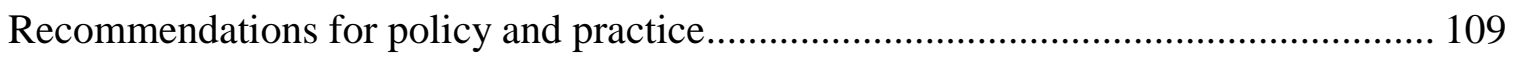

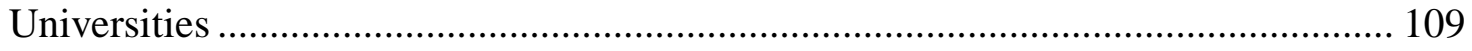

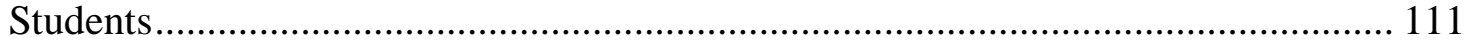

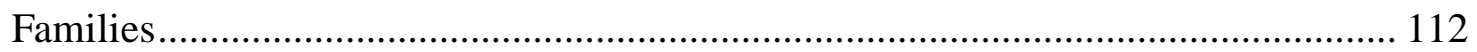

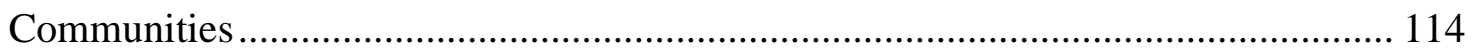

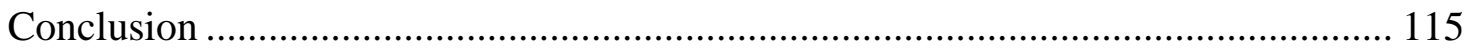

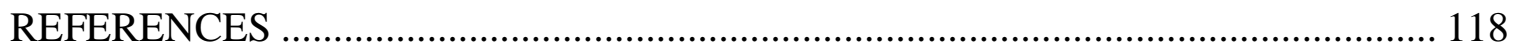

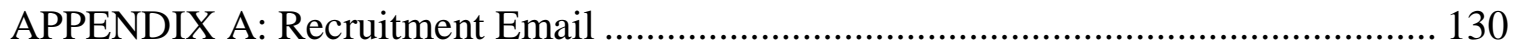

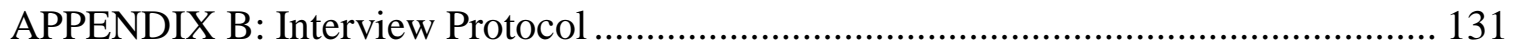

APPENDIX C: Focus Group Protocol................................................................. 134

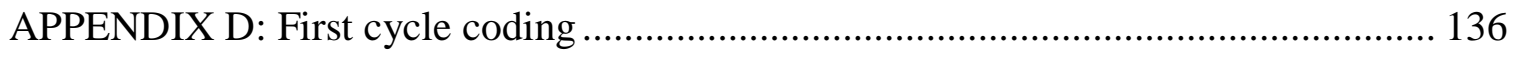

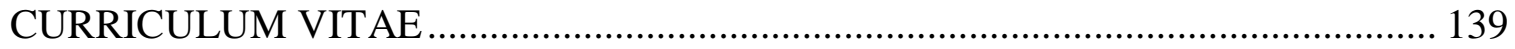




\section{LIST OF TABLES}

TABLE

PAGE

1. Types of transition definitions.................................... 36

2. First cycle coding definition...................................62

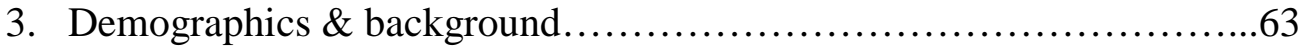

4. Proposed new 4S system....................................119 


\section{LIST OF FIGURES}

FIGURE PAGE

1. Appalachian Regional Commission County designation................6

2. Cycle of educational impact on economic development................13

3. Process of moving in, moving through, and out of transition.............38

4. Appalachian Regional Commission identified AKY counties..........60

5. Thematic map showing self.....................................81

6. Thematic map showing support..................................85

7. Thematic map showing situation................................94

8. Thematic map showing strategies................................. 100

9. Transitional phases diagram..................................110 


\section{VIGNETTE:}

"So even though there are twenty thousand students here...(With) engineering being a smaller program at the university it still has a very homey feel very small town feel. In terms of the workload it was a very different thing, so coming to college was a big slap in the face. And I've never had a problem introducing myself and making friends that wasn't difficult. But I think not having family close was difficult in terms of socially... that and trying to make friends is one thing but building a support system is another. And my parents are supportive...it's just difficult when they are two and half hours away.”Mary 


\section{CHAPTER 1: INTRODUCTION}

Graduates in Science, Technology, Engineering, and Mathematics (STEM) are critical for the United States to remain competitive and innovative as the global economy continues to accelerate (Bhef, 2010). In 2014, the National Science Foundation (NSF) reported an ongoing and strong demand for college graduates with degrees in the STEM fields. Although enrollment within university STEM programs continues to grow, the current rate of growth is not enough to fulfill industry demands (College Recruitment Practices, 2017; Mulvenon \& Robinson, 2014). The previous Kentucky governor, Matt Bevin, has stated the need for Universities to focus more on STEM education and articulated his vision for the Commonwealth of Kentucky to become the engineering hub of the country (Seltzer, 2017). This concentration on STEM education is shown by the state of Kentucky's recent performance-based funding model for higher education, where a strong emphasis has been placed on enrollment in, and completion of, STEM degree programs.

The need to grow enrollment within the STEM discipline led universities to expand their recruiting efforts into locations that have historically been overlooked or received little attention, such as rural secondary schools. Over $50 \%$ of public schools across the United States are located in rural areas (The National Center for Educational Statistics, 2003). During this same time period [2010], the Kentucky Appalachian Region Commission reported a population of 1.2 million individuals; roughly one-third of the entire state lives within the rural Appalachia region of 
Kentucky (ARC, 2010). In addition, 32\% of the Kentucky Appalachian (AKY) population is under the age of 24 , which is important as the traditional recruiting pool for college students comes from this age group. The Appalachian region is also home to roughly $9 \%$ of all individuals under the age of 24 in the Commonwealth of Kentucky. Given the large number of individuals living in AKY, especially those under the age of 24, it is not surprising that many universities within the state have identified this region as a strong potential recruiting location.

The National Science Foundation (NSF) reported in 2014 that there is an ongoing and strong demand for college graduates with degrees in the STEM (Science, Technology, Engineering and Mathematics) fields. Recent trends have shown a decrease (1\% for students age 18-24) in enrollment within higher education, however during the same time STEM enrollment saw an increase of 2.2\% for this age group (Schaffhauser, 2018). The US awarded 233,000 STEM degrees across all levels and disciplines in 2007; this is far fewer than other developed counties; for example, China out produced by (46.7\%); South Korea (37.8\%); and Germany out produced (28.1\%) more STEM degrees then the US (Forum, 2010). Producing STEM graduates has become a national priority, both to meet the job market needs and to create opportunities for US workers.

Over the last decade, scholars have examined barriers that have limited underrepresented students in STEM education (Diekman et al., 2010). As U.S. higher education institutions struggle to produce STEM graduates, there is a need to understand what factors contribute to increasing success within these majors, beginning with the student's transition. There is a vast amount of research surrounding the STEM education and success variables for the general population: ACT scores, gender, and high school 
GPAs are indicators of success (Johnson, 2012). However, there still exists a need to analyze best practices regarding how specific subpopulations can be successful within STEM education (Yoder, 2012).

Figure 1

Appalachian Regional Commission, Appalachia county designation

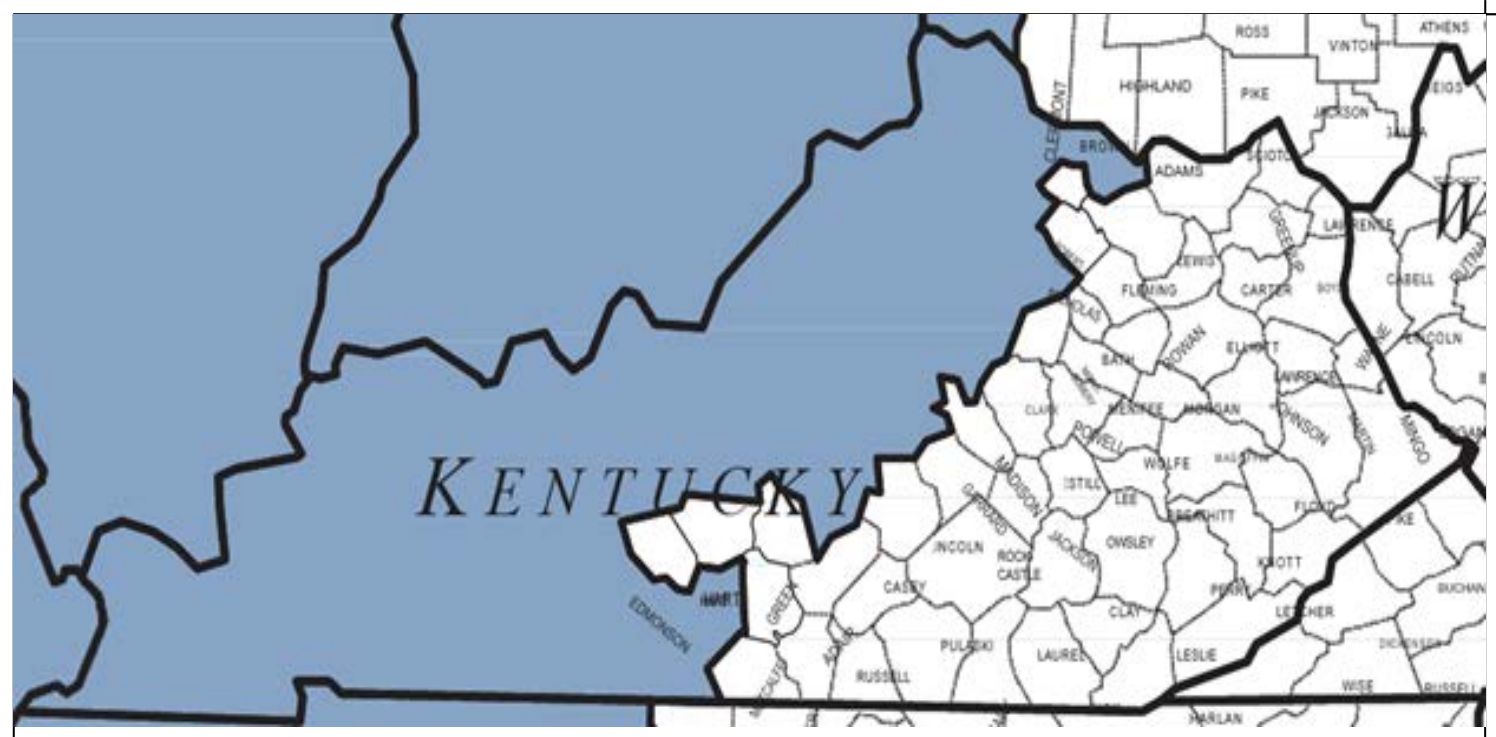

Kentucky Appalachian Counties: Adair, Bath, Bell, Boyd, Breathitt, Carter, Casey, Clark, Clay, Clinton, Cumberland, Edmonson, Elliott, Estill, Fleming, Floyd, Garrard, Green, Greenup, Harlan, Hart, Jackson, Johnson, Knott, Knox, Laurel, Lawrence, Lee, Leslie, Letcher, Lewis, Lincoln, McCreary, Madison, Magoffin, Martin, Menifee, Metcalfe, Monroe, Montgomery, Morgan, Nicholas, Owsley, Perry, Pike, Powell, Pulaski, Robertson, Rockcastle, Rowan, Russell, Wayne, Whitley, and Wolfe

Note. By author

Recruiting students is one step in the process of fulfilling the need for a STEM-trained population; ensuring that students have the opportunity to be successful in these programs is the next and vital step to meeting the emerging needs of industry (Mulvenon \& Robinson, 2014). Although there are many factors that lead to an individual's success in college, the transition into college has the potential to be the first major step. Ample literature exists on students transitioning into college as well as factors that lead to success in obtaining a STEM degree, however, 
students from the Appalachian region are highly underrepresented in these studies. The few existing studies, that focus on both STEM majors and rural students, are based on quantitative analysis of success factors such as college-going culture, social support, etc. (Hudacs, 2017). There is a lack of research that focuses on AKY individuals' lived experience during their transition into college through the lens of qualitative research. If universities continue to increase their recruitment efforts within this subpopulation, it becomes imperative to also research their unique needs as incoming students in order to improve their retention and persistence to graduation. Additionally, we must understand what strengths students possess which they can leverage to positively impact their transition (Yosso, 2005). Understanding the experience of AKY students' transition into college and journey throughout their STEM studies will assist in providing higher education practitioners with the tools, programs, and interventions to assist these students throughout their college career.

\section{Purpose Statement}

The purpose of this qualitative study is to examine the lived experience of rural (AKY) students studying STEM at an urban university. This study seeks to investigate

the unique experiences of AKY students transitioning to college and the common factors between their transitional experience into college while addressing compounding factors of a rigorous STEM major and a new cultural setting at an urban institution.

\section{Research Question}

The central question for this study is "How do rural AKY students experience their transition into college, while pursuing a STEM degree at an urban university?” To answer this overarching question the researcher created the following specific research questions: 
Q1: What individual factors influence the transitional experience?

Q2: What factors of the individual's support system (family, hometown and university) influenced the transitional experience?

Q3: What factors within an individual's situation influenced the transitional experience?

Q4: What factors within the individual's strategies influenced the transitional experience?

\section{Definition of Terms}

The following term definitions are used by the researcher for this study. Adaptation: adaptation to transition is a process during which an individual moves from being totally preoccupied with the transition to integrating the transition into his or her life

Appalachian Kentucky: The 53 counties designated by the Appalachian Regional commission as Appalachian counties within the state of Kentucky College student: an individual enrolled part time or full time within a higher education institution

Self: personal, demographic and psychological resources of one's self which influence an individual's viewpoint and belief system

Situation: various factors which influence the transition (trigger, timing, control, role change, duration, previous experience with a similar transition, concurrent stress and assessment)

STEM: science, technology, engineering, math

Support: social support in the form of: relationships, family units, networks of friends, institutions and communities 
Strategies: coping responses to the transitions (modify the situation, controlling the meaning of the problem, and managing the stress)

Transition: an event or non-event that results in a change of assumptions about oneself and the world which then requires a corresponding change in one's behavior and relationships (Schlossberg, 1984)

Transition to college: 1-2 semester timeline within college at the specific institution

\section{Significance of the Study}

This study provides significance both at a micro and macro level by providing a voice to a subpopulation who are often forgotten; however, due to recent enrollment pressures, rural students have become a targeted recruitment population for universities. This body of work can provide scholarly significance through a qualitative lens by focusing on the AKY subpopulation, a population often overlooked by researchers. Additionally, this study can provide practical significance to the AKY student, AKY communities, urban universities, administrators/practitioners, and policymakers on supporting this subpopulation, leading to increased graduation rates and ultimately increasing the economic development of the state.

Transitioning to college has potential implications for students in their college success, persistence in their higher education journey, and following their planned career trajectory (Gale and Parker, 2014; Hultberg et al., 2008; Leese 2010; Tinto, 1993). The transition to college is a complex, highly individualized experience, which can be heavily influenced by individuals' subpopulations and intersectionality (McMillan, 2014). Although research has made great strides, there 
still exists a significant gap on transitioning to college while majoring in a rigorous discipline such as STEM, specifically through the lens of the Appalachian student perceptive. As universities strive to increase enrollment, and legislators look to increase economic development in rural communities, this study can further provide success by enhancing their understanding of AKY students' transitional experiences, thus assisting in the development of supporting interventions and policies. This study strives to fill the identified gap by providing a voice to AKY students and analyzing common threads between their transitional experiences.

\section{Practical significance to AKY students}

This research has the potential to benefit AKY students by allowing individuals to gain an understanding of expectations in transitioning to college while studying STEM, as well as the strategies needed to manage through the transitional process. AKY students who are able to transition into college successfully have the potential to increase the likelihood of obtaining a STEM degree, launching into an in-demand career field, and increasing their economic mobility. The ability to successfully transition into college and obtain a degree provides an opportunity to break the poverty cycle that AKY students are likely to have experienced while growing up in rural Appalachia.

\section{Practical significance to universities}

AKY students can inform universities of their transitional experience, allowing practitioners to enhance opportunities for increased success through this research. The AKY student voice is important as universities are under extreme pressure to increase student enrollment, retention, and graduation rates (Mulvenon \& Robinson, 2014). As state funding continues to decrease and available funding is 
closely linked to enrollment numbers, universities will feel the pressure to recruit more students. This research can arm universities with the knowledge necessary to build and offer programming to AKY students increasing the likelihood of success in college. Within the Commonwealth of Kentucky, an increase in retention and graduation rates of STEM majors from AKY students would also lead to an increase in enrollment funding for the university by the state.

\section{Practical significance to practitioners}

Results from this study have significance for practitioners through the opportunity to better understand the subpopulation of AKY students by highlighting common aspects of their identities within an urban college setting. As it stands, this subpopulation has the potential to experience oppression by peers and university administration, stemming from a lack of understanding of AKY students by the university community (Rendon, 1995). By viewing AKY students as a subpopulation, and understanding their unique characteristics and challenges, practitioners can develop interventions targeted to assisting with the transition to college, address the aforementioned oppression, and provide support throughout their time in higher education. This will allow practitioners such as student affairs professionals and academic advisors to engage AKY students in conversations that address the coping mechanisms needed for the successful transition of this portion of the student population.

\section{Practical significance to policymakers}

This research has practical significance to influence policymakers, ensuring that future legislation is developed to allocate funding to AKY recruits and motivate higher education facilities to assist these students by supporting their 
success. If higher education institutions are able to increase STEM degrees within the state through the rural community population, then universities have the opportunity to positively impact the economic development of the state. Lastly, an increase in college-educated talent has the potential to increase the desire for companies to relocate into the state, bringing more jobs, and higher skilled (therefore higher paid) jobs to the areas, which would enhance the economic development of the state.

Figure 2

\section{Cycle of educational impact on economic development}

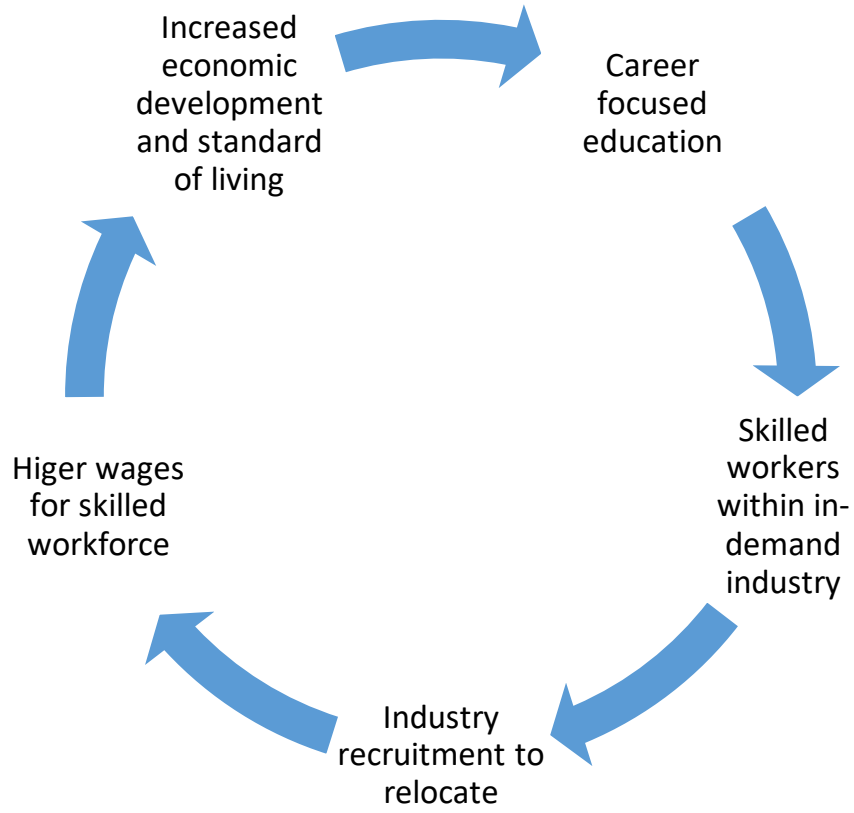

Note. By author

\section{Rationale for the study}

This study strives to fill gaps in the existing literature by providing a voice to the AKY students' lived experiences of transitioning to college while studying STEM at an urban institution. Through a qualitative lens this study can assist the AKY students, AKY communities, university administrators, and policy makers to create an environment 
where AKY students have a positive experience transitioning to college. Through the lens of Schlossberg's transitional theory (1981), the research examines the common threads of coping during the transition of moving in, moving through, and moving out stages of an AKY student's college transition. This study can add a dimension to the body of research examining college success, college transition, and rural student transition into college. At the time of this study, no qualitative work examining the lived experience of AKY students transitioning into college and studying STEM is known to exist.

With the current targeted recruitment and lack of transitional research for AKY students into a STEM major, stakeholders must understand the importance of early intervention and support of this subpopulation to increase retention and ultimately degree completion. The themes from this study provide significant information for both AKY students, AKY communities, university administrators, and policy makers within the state. First, the themes of this study can provide AKY students guidance with knowledge on strategies that will assist in a smoother transition into college. AKY students having realistic expectations and developing the necessary skills will provide the opportunity to influence a positive transition. Second, AKY communities can utilize these results to create programming within the K-12 schools and within the community to support and prepare students to attend an urban institution while studying a rigorous program such as STEM. Third, the study results could assist university administrators in recognizing AKY students as a subpopulation with specific known barriers. This study could reveal these barriers and assist in the development of interventions to support AKY students' transitioning into college within STEM disciplines. Findings can guide policymakers to create a college-going culture and support the educational attainment of persons from the AKY region. Finally, this study has the potential to give a voice to students who have 
lacked the ability to share their experiences and feelings while making the most important transition of their young adult lives.

\section{Organization of the study}

The researcher has organized this paper into five sections. Chapter 1 introduces the study, describes the purpose and its significance. Within chapter 2, the literature review, examines the various factors within the study and the theoretical framework utilized within the study. Chapter 3 methods described the research approach, and participant information. Analysis of the data collection through cycle one and cycle two coding is found within chapter 4 . Chapter 5 describes potential implications and the need for additional research. 


\section{CHAPTER 2: LITERATURE REVIEW}

This chapter examines the literature that explores the factors of AKY students transitioning into college while studying a STEM discipline. The chapter is structured in the following manner: (a) factors which influence the students' transition into college, (b) STEM education, (c) college attainment by rural Americans, and (d) review of the theoretical framework.

\section{Student college choice}

Retention begins before the student steps foot on campus; however, a student's retention is partially influenced by a student's college choice (St. John, Paulsen, \& Starkey, 1996). Research suggests that parents’ educational expectations for their children to be the strongest influence on a student's decision to attend college and what type of college they ultimately attend (McCollough, 2011). St. John, Paulsen and Starkey (1996) discuss in their research surrounding college choice and persistence reasons why a student chooses a college can have both positive and negative influences on their persistence. Specifically, those who selected a college/ major based on work-related outcomes saw a positive correlation with persistence. However, students who selected a college based on low tuition saw negative persistence, which is important as many students from AKY have a higher potential of living in poverty and thus focusing on attending the cheapest university. Additionally, AKY parents are less likely to have a college degree and potentially be less supportive of their children pursuing a college degree. Without family and community members with college degrees, students in the community 
lack role models to aspire to become through educational attainment. As we examine underrepresented rural populations with limited knowledge and access to certain higher educational institutions, specifically those in urban settings, it will be vital that we remember the lens in which many within this population find as their reality.

\section{Transition to College}

There is little research on how subpopulations experience their transition into college; the literature involving subpopulations transition to college focuses on African American, first generation, and veterans (William, 2011). A college transition is defined as a time where an individual gains freedom from parents and an increase of connections to others in the community, such as peers and institutional support staff within a university setting (Mattanah, Ayers, Brand, Brooks, Quimby \&McNary, 2010). Additionally, the transition to college is the beginning of a person’s educational journey (Mattanah, Ayers, Brand, Brooks, Quimby, \& McNary, 2010). Students entering college have the potential to experience a challenging transition (McCoy,2004; McGhie, 2017; Postareff, et. al, 2017). These challenges can include: the lack of cognitive development (Holmstrom, Karp, \& Gray, 2002) and social/ family support (Gall, Evans \& Bellerose, 2000), as well as the inability to adapt in to the college setting (Wang, 2014). These challenges have the potential to influence the experience of the transition, which could affect one's success within college and potentially impact the economic development of the state, as well as the workforce demands. A vast number of researchers have suggested that college success is determined by the 
student’s first year experience (Smith \& Bracken 1993, Tinto, 1993; Upcraft \& Gardner, 1989; Wolcott, 2006). A successful transition into college relies on both the academic and non-academic factors within the individuals (Venezia \& Jaeger, 2013).

There is a variety of reasons as to why a successful adaptation of transitioning to college is important. Gardner \& Siegel (2001) suggests that the first weeks in a new university will determine how seriously a student will take their college experience. College is a time for new culture, friends, support systems, and daily agenda, along with a new sense of independence. Attending a university and embracing changes can prove to be a stressful period for new students. (Bouteyre, Maurel \& Bernaud, 2007). Students who enter college have the potential to experience a difficult transition; one where an individual is faced with a number of challenges: lack of social network, increased school work and negotiating the "temptations" of a college environment (Mattanah, Ayers, Brand, Brooks, Quimby \& McNary, 2010). Since the transitional experience into college has the potential to be a retention factor within postsecondary education, there is value in gaining insight through research. A focus on the transitional experience is vital considering the 2016 fall persistence of first-year college students into their second year within US institutions averaged $73.4 \%$; thus $27 \%$ college freshmen are not returning to their university after their first year (National Student Clearinghouse: research center, 2018).

\section{External transitional factors.}

Gardner (2001) suggests that the first weeks in a new university will determine how seriously a student will take their college experience. During this 
time is when an individual gains freedom from parents and an increase of connections to others in the community, such as peers and institutional support staff (Mattanah, Ayers, Brand, Brooks, Quimby \&McNary, 2010). External factors include the campus culture, support, financial needs, family issues, and academic preparations. These external factors have the potential to influence the transition either positively or negatively.

\section{Internal transitional factors.}

College is a time for new culture, friends, support systems, and daily agenda- along with a new sense of independence. Attending a university and embracing changes proves to be a stressful period for new students. (Bouteyre, Maurel \& Bernaud, 2007). A successful transition into college relies on both the academic and non-academic factors within the individuals (Venezia \& Jaeger, 2013). Internal factors such as one’s self esteem, grit, coping mechanisms, and determination have the potential to influence their ability to adapt throughout the transition.

\section{Student academic and social transition}

The literature has very few studies which have investigated the impact of transition on the general well-being of new students (Aspinwall \& Taylor, 1992). Students who adapt poorly within their transition could be struggling academically or socially; or a combination of both (Venezia \& Jaeger, 2013). Social aspects of college include social and support networks, daily routines, and the campus environment. It has been suggested that students with college expectations that are negative, such as anxiousness and fear of failure, have more difficulty transitioning 
to college; while those students with positive expectations, such as excitement, have an easier time transitioning to college (Mattanah, Ayers, Brand, Brooks, Quimby, \& McNary, 2010). Support which includes family, peer, university faculty, staff, and student organizations have the potential to influence a student's positive or negative experience as they transition to college. Among support, parental support has been found to be an important form of social capital that facilitates the transition from high school to college (William, 2011). For students whose new environment is not located near their family, the social adjustment has a major impact on the adjustment process (Nelson et al, 2007). Individual attention must be given to each student regarding their needs to successfully transition into a post-secondary setting (Gall, Evans, \& Bellerose, 2000).

Social adaptation is not the only transitional focus that a student must face in transitioning to college; academic expectations are another. Academic preparedness has been well studied as a key to persistence and retention (Camara, 2013), which represents a key measure to success. However, there is a gap in research regarding how academic preparedness directly influences the student's ability to transition successfully in college (William, 2011). William's research states that the quality of high school academics is critical to making a smooth transition to college. Although not widely studied, this does present a potential barrier for students from Appalachia who are at a higher risk for poorer academic preparation for college.

Adapting to college academically and socially: social network, work, and negotiating the temptation of a college environment are potential challenges students face during their transition to college. There is a research gap when it 
comes to the transition to college of vulnerable populations and the effects of transitioning to college.

\section{Academic Preparedness}

Academic preparation has been identified as a major key to retention within college (Perna, 2005; Reid, M.J. \& Moore, J.L.; 2008; Rendon, 1995; \& Tinto, 1993). Academic preparation influences a student’s self-esteem, outlook, commitment and resilience leading to persistence within a postsecondary institution, which is why academic preparation must be included within the literature regarding the transition to college. Specifically, math preparation is an indicator of success within college (Shute, Hansen, Underwood, \& Razzouk, 2011). Math preparation has the potential to predict success as many STEM majors require high level math knowledge throughout the curriculum. These factors create barriers for students from AKY due to lack of access to resources and high-quality advanced courses needed to prepare them for the rigorous academics of STEM education. Successful transition to college includes high school academic preparation, specifically mathematics and the ability to develop learning strategies. The Appalachian Regional Commission reports that the AKY region has the lowest post-secondary educational attainment (14.3\%) in the Commonwealth of Kentucky. In 2014, 36.4\% of AKY high school seniors tested as college ready according to the math section on the ACT. This data point is important as college ready math is a minimum at many institutions to take calculus, which is understood to be first math course for students entering a STEM major (Mokher, 2011). Tinto (1993) describes the importance of high school preparation and how the intention of students plays a major role in retention at a university. If a college degree is the pathway to the 
middle class in America (College Affordability and Completion: Ensuring a Pathway to Opportunity, 2019) and we know that AKY lacks educational attainment and academic preparation, then there's a possibility that this population of students transition to college could face greater barriers. If federal programs and state legislation are focused on college degrees within STEM, we need to examine how students within STEM majors have experienced this transition as it is the cornerstone of persistence in attaining a college degree.

\section{Cognitive Development}

Cognitive development of an individual has the potential to influence the transitional experience into college either positively or negatively. Adjustments to life events are influenced by the individual's cognitive ability and coping behaviors (Gall, Evans, \& Bellerose, 2000). Factors which predict short-and-long term adjustment to the university transition include well-being, life events, social support, coping behaviors, and cognitive appraisal (Gall, Evans, \& Bellerose, 2000). Cognitive development is also important within the transition as an individual has the potential to view college as an opportunity to be self-sufficient outside their family circle if their confidence is strong enough (Holmstorm, Karp, \& Gray, 2002). One's individual self has the ability to impact how they view, approach, or manage the transition into college. The ability to take ownership and determine the approach to view and manage the transition has the potential to influence an individual's experience.

\section{Social/ Family Support}

In addition to the individual, social support, both within the university and the individual's family, plays a role in the transition to college (Lazarowicz, 2015; 
Mattanah et al, 2010). Families understanding of the stress of college and providing support can assist with a student's ability to cope in a timely manner and thus transition more effectively (Gall, Evans, \& Bellerose, 2010). Families and home community members have the potential to positively or negatively influence the students' ability to gain access regarding college, based on one's familial capital (Yosso, 2005).

Yosso (2005) refers to familial capital as one of the six factors which makes up an individual's community cultural wealth; which she argues influences a students' persistence in college. The six factors include: aspirational, familial, social, navigational, resistant, and linguistic capital. Aspirational capital refers to the ability to maintain hope and dreams for the future in the face of potential barriers. This could be a student scoring below average on a math course and determining whether they still have dreams to stay in their selected major. Social support can influence aspirational capital as they can positively or negatively encourage the individual's dream. Familial capital, as discussed previously, is the social and personal human resources students have in their precollege environment. For AKY students, this could be their home community which potentially doesn't support students going to college. AKY students could possess negative familial capital because of the low higher educational attainment within this region. The third capital is navigational. This is the student's skills and abilities to navigate "social institutions". Although, one could argue this fits within the self or individual realm of the transition; the university has a responsibility to provide the skills and develop the ability for every student to be able to successfully navigate the institution. The fourth capital is linguistic. Linguistic is described as the ability 
to develop communication skills through various experiences. Social/family support plays a role in developing the communication skills to navigate college and the ability to communicate with faculty as well as peers. Social capital is defined as peers and other social contacts; as well as how a student utilizes these contacts to assist in the access and navigation of their college experience. This would be a student developing a friend/ support group within college which positively or negatively impacts their ability to navigate college. AKY students may have a disadvantage in developing positive social groups to navigate college as there's the potential to be the only person from their high school attending the university. The last capital is resistance which refers to communities of color in securing equal rights and collective freedom.

Support has the potential to influence a student's positive or negative experience as they transition to college. Among support, parental support has been found to be an important form of social capital that facilitates the transition from high school to college (William, 2011). For students whose new environment is not located near their family, social adjustment has a major impact on the transitional process (Nelson et al., 2007).

Programming by the university to build community and social support is vital to a successful transition and, ultimately, retention to the university (Mattanah et al., 2010). The transitional experience of first-generation students indicates that support among teachers and other administrators assisted in a successful transition into college (Wang, 2014). The size and helpfulness of one's social support also has an impact on the transition process (Gall, Evans, \& Bellerose, 2010). Common themes such as family focused and financial concerns center on support from 
family and the university, such as the concern with time constraints, job related constraints, perception of institutional support, and faculty interaction, emerged from an examination of male drop outs and their transitional experience reentering higher education (Powers, 2010). These studies highlight the importance of support both from the family and the university as an approach to manage the transition into college.

\section{Adaptation}

The ability to adapt within a situation and manage transitions has been identified as a factor that assists in a successful transition into college. Notably, coping mechanisms were found to be the most important factor throughout this transition for veteran students (Wheeler, 2012). Veteran students who demonstrated balance between one’s self, their support system, situation, and strategies were able to manage a more successful transition into college (Wheeler, 2012).

Transitioning into college has the potential to be marked by changing roles, new challenges, and increased responsibilities (Conley et. al., 2014). The ability to adapt includes the potential to manage and cope with ambiguous situations such as adjusting to college (Venezia \& Jaeger, 2013). Students should use coping strategies to reframe or work through the transition and look for opportunities to acquire additional resources to assist with issues throughout their experience (Folkman \& Moskowitz, 2000). Expanding on the use of coping strategies, the transitional experience of freshmen commuter students transitioning into college suggests that a student's ability to adapt to new situations, pursue goals, and overcome obstacles are key components in adapting to college (Clark, 2005). The ability to adapt is essential in transitioning to college, but how can administrators 
and practitioners ensure that students from subpopulations and disadvantaged situations have the opportunity to acquire the skills needed? Subpopulations have the potential to face additional barriers and may lack the coping mechanisms or the need to utilize one strategy more intensely than others researched by Schlossberg.

\section{STEM Education}

One major transitional success factor of STEM students is the support of academic quality and learning environment within their high school (Xu, 2015). Xu discusses themes including higher GPA that increased success of transition into STEM majors. Specifically, high GPA and levels of determination lead to stronger academic

pursuit. Although valuable, this quantitative study lacked understanding the voices of participants and their experience as they entered college while balancing the academic pressures of studying STEM. The American Society of Engineering Education (ASEE) published a report dedicated for universities to assist with retention of Engineering specific students in the areas of tutoring/mentoring, student programs, financial aid, academic enrichment programs, student research, curriculum and class enhancements, institutional/educational research and change in institutional/departmental policy (Yoder, 2012). Moreover, research regarding success within STEM overwhelmingly recognizes that academic preparation in math and science are key factors to success (Engberg \& Wolniak, 2013; Li, Swaminathan, \& Tang, 2009). However, the literature lacks understanding of STEM education and how transition affects the students; especially those underrepresented populations who are transitioning in multiple facets as they come to college.

\section{Rural \& Appalachian Communities}

\section{Rural communities}


Rural K-12 schools account for $53 \%$ of all educational institutions across America (National Center for Educational Statistics, 2003). However postsecondary institutions may not be prepared to assist in the transition of students from rural communities.

Rural communities throughout the United States differ and have a variety of issues associated with their location such as high poverty rates, high unemployment, and low educational attainment (Edington \& Conrad, 1981). Edington and Conrad (1981) also state that educational resources and quality vary from rural community to rural community. This reinforces the need to review literature regarding rural communities but also to ensure that research regarding specific communities occurs in an effort to support that specific community breakdown their barriers.

Rural students were less likely than urban students to attend college in general, but those who did attend graduated at the same rates as urban students (Gibbs, 1989). Additionally, rural students were significantly more likely than urban students to graduate from rural post-secondary institutions (Gibbs, 1989). If universities assist students from rural communities in their transition there is the potential to increase their graduation rates (Gibbs, 1989).

Cultural capital of familial support and academic preparedness are suggested to play a larger role in the success of rural students in college than where they are from (Hudacs, 2017). Although these factors may have played large roles in the success of a student transitioning to college; the factors of poor academic preparedness and lack of family support are common factors within rural communities (Gibbs, 1989). Ganss research describes rural students as having an 
increase in unexpected negative emotional transition, lack of social involvement, and rural students being overwhelmed with the diversity of their new college community. All of these factors lead to consciousness of their rural identity. This supports the importance of understanding the specific experience of subpopulations which is vital to the success of both their transition and within the University.

\section{Appalachian Communities}

The (AKY) region is a small set of counties within eastern Kentucky, which is part of the larger Appalachia region spanning multiple states. This specific rural community has been identified as a subset population which lacks research on college transition, and yet is quickly becoming a hot-bed for college recruitment efforts. This deficiency in research is further highlighted when specifically examining AKY students' transition to an urban institution and studying STEM.

Understanding the need for additional research within this population comes from an understanding of the economic factors and cultural norms; the norms that influence encouragement of educational attainment. The AKY region struggles with low economic resources and the ability to transform into a work force that requires college degrees. In general, the AKY region has higher poverty levels (25.9\%) compared to the state of Kentucky as a whole (18.8\%). Of the entire Appalachia region (including the AKY region), 16.7\% live below the poverty level (Poverty Rates 2012-2016, Appalachian Regional Commission report). In 2015, the AKY average income was $\$ 19,204$ compared to the rest of Kentucky at $\$ 29,051$ (County Economic Status, Fiscal Year 2016, Appalachian Regional Commission report). The national poverty level for a family of four within the United States in 2015 was \$24,250 (Federal Poverty Line Guidelines, 2015). The poverty and economic 
distress within the Appalachia region are correlated with parents' focus on basic needs (food, water, and housing), low levels of college attainment, and lack of education in general (The Rural and Appalachian Youth and Families Consortium, 1996). Because of the economic state of the region, parents tend to view higher education for their children as out of reach; this is especially influenced by the high financial cost of such endeavors (Bryan \& Simmons, 2009).

Poverty and poor academic readiness are found in greater numbers both in rural communities in general and specifically in the (AKY) region. These factors, academic preparedness and poverty, are particularly impactful for Appalachian KY residents where the average household income is typically below the poverty level, educational attainment is lower than the national average, and access to the internet/ academic prep can be limited (National Education Center, 2013). The AKY region is likely to face many barriers: economic, poverty, unemployment, and lack of role models in their journey toward educational attainment. These factors among rural Appalachian residents have the potential to be enhanced within the Appalachian region as a individuals' identity is directly connected with their communities.

The AKY community lacks a college attainment culture with only $14.3 \%$ of the population having obtained a postsecondary degree (Appalachian Regional Commission Report, 2016). AKY students who decide to leave home and attend college have the potential to experience isolation, loneliness, and low self-esteem given the lack of support within their community. Community identity plays a strong role in one’s identity in Appalachia (Bryan \& Simmons, 2009; Davis-Kean, 2005; Family Relation 45(4), 1996; \& Pulcini, 2016); thus, community support for college attainment is a vital piece in success—one that individuals transitioning will 
need to obtain along with becoming self-sufficient, through support of the university, and support of their family. Increasing community support for a college-going culture can assist AKY students’ mindsets in attending not only a college but also studying STEM at an urban institution.

In addition to poverty being a factor within the AKY region, social and cultural factors are prevalent. The Appalachian region as a whole has a $45 \%$ college going rate, whereas the US in general has a rate of 63.3\% (Schwartz, 2004). Schwartz (2004) states that 17\% of Appalachian adults (25 years or older) have a college degree. Parents, who provide influence over a child's pathway, lack support for students to attend college due to lack of experience and financial resources (Niu, 2017; Schwartz, 2004). As AKY holds a strong connection to their community identity and the region as a whole lacks experience within higher education and college attainment, children then lack role models, and encouragement to envision college attainment within their life trajectory.

Socialization within Appalachia is believed to be fostered through a closeknit family (immediate and extended), many of whom live in the same town and, at times, on the same property (The Rural and Appalachian Youth and Families Consortium, 1996). Immediate families, extended families, community, family involvement within the community, parental support, and perception of resources are major influences on Appalachian people (The Rural and Appalachian youth and Families Consortium, 1996). Due to needs and accessibility, the community influences the acceptance of low paying service jobs, low educational attainment, as well as satisfaction with only the most basic resources (The Rural and Appalachian Youth and Families Consortium, 1996). Negative community 
opinions surrounding higher education has the potential to negatively affect an AKY student's transitional experience, if they do make the choice to attend college.

As most students from AKY are also first generation there's the potential for greater barriers due to a lack of realistic expectations regarding their transition to college such as academic, social, cultural, financial, and personal independence issues. Gibbs' (1989) does provide insight to the need for interventions and programs designed to support rural students, including Appalachian students, that could potentially lead to increased educational attainment, reduced unemployment, and increased equity within the state of Kentucky. Additionally, legislators should also look at the potential of expanding STEM programs into rural universities.

\section{Theoretical Framework}

Of the many theories focused on transitioning to college, Schlossberg's transitional theory is one perceptive which has been widely accepted by higher education scholars. The Schlossberg's transitional theory has been accepted within higher education research community as a model that assist both researchers and practitioners in understanding factors in a student's transition into college as seen in Evans (2010), Lazarowicz (2015), Powers (2010), and Ryan et. al. (2011). These researchers have utilized Schlossberg's transitional theory in a variety of subpopulations (first generation, black males, veterans, etc.) to explore their experience of transitioning into college. This theory was selected as the theoretical lens for three major reasons: a) the theory has been widely accepted as relevant within higher education research and practice; b) this theory describes the process of moving through the transition and strategies needed to successfully manage the 
transition; and c) Schlossberg's framework was developed to facilitate an understanding of the transition. Through Schlossberg's transitional theory lens, this study hopes to gain an understanding of AKY students' transitional experience while studying a STEM discipline within college and providing elements to support future students in the transition.

\section{Schlossberg's Transitional Theory}

Schlossberg’s (1981) transitional theory was originally developed to examine the transition of adults from career to retirement, providing insight into the factors which influence the transition, individual, and degree to which the individual is impacted by the transition at that particular time (Powers, 2010). Transition is defined as any event or non-event that results in the change of relationships, routines, assumptions and roles (Schlossberg, 1981). Expanding on the theory, Schlossberg examined the various aspects of moving into, through, and exiting a transition (Chickering \& Schlossberg, 1995). Transitions vary from person to person; they aren't determined by the society in which the event occurs (Schlossberg, 1984), but through the individual's perception of the event as the key component in defining a transition (Chickering \& Schlossberg, 1995).

Schlossberg's (1984) theory discusses the importance of coping mechanisms within a transition and introduce four specific approaches to enhancing a transitional experience, known as the $4 \mathrm{~S}$ system.

\section{Transitional types.}

According to Schlossberg, there are three types of transitions: anticipated, unanticipated, and nonevents. An anticipated transition is a change in life that is expected. An example is a person who graduates high school during spring of their 
senior year, expects to attend college in the fall, and does so. Unanticipated transition is a change in life which was not anticipated. An example of an unanticipated transition is a person who graduates high school spring their senior year, expects to attend college but is not accepted into their top choice forcing the person to attend a less desirable university. A nonevent transition is a change in life that is expected but didn't happen. For example, a senior in high school plans to attend college but is not accepted and thus does not further their education past high school. Research suggests that these three transitions exist as an individual experiences change within a person's life. The impact of each transition depends on how it alters an individual's life (Schlossberg, 1984).

Table 1

Types of transition definition

\begin{tabular}{|l|l|l|}
\hline Types of transitions & Definition & Example \\
\hline Anticipated & Change in life that is expected & $\begin{array}{l}\text { Graduates high school, } \\
\text { expects to attend college and } \\
\text { does }\end{array}$ \\
\hline Unanticipated & $\begin{array}{l}\text { Change in life which is not } \\
\text { expected }\end{array}$ & $\begin{array}{l}\text { Graduates high school, is not } \\
\text { accepted in to first choice } \\
\text { college and must attend } \\
\text { another university }\end{array}$ \\
\hline Nonevent & $\begin{array}{l}\text { Change in life that is expected } \\
\text { but doesn't happen }\end{array}$ & $\begin{array}{l}\text { Graduates high school, } \\
\text { expected to attend college but } \\
\text { is not accepted and doesn't } \\
\text { further school past high } \\
\text { school }\end{array}$ \\
\hline
\end{tabular}

Note. By author

\section{Process of a transition.}

Within each type of transition, a person experiences three phases: the moving in, through, and out of the transition. These stages exist within all events or non-events, however the time spent within each stage is up to the individual (Evans, 2010). The beginning of a transition is classified as "moving in”. Moving in 
occurs when a person is confronted with a transition or change; they leave one known context and enter a new one (Komives \& Brown, n.d., p.4). This could include leaving home and moving into a residence hall or entering orientation. The middle of the transition is classified as "moving through" (Evans, 2010). Moving through is defined as the day to day management of the new context or life experience (Komives \& Brown, n.d., p.4). Komives and Brown suggest that moving through the college transition could include choosing a major, moving into the residence hall, time management, friendship development and finding one’s place within the new community. This process varies in time, determined by the transition and individual within the transition. The end of the transition is classified as "moving out" (Evans, 2010), which is defined as the end of the transition (Komives \& Brown, n.d., p.5). This is the point in which a person has adapted into the new situation and is ready to move into a new transition; this could be adjusting to the new life at college and beginning to engage in the community through joining organizations or applying for graduation. To successfully move out of the transition one must have a strong foundation of approaching the change, coping, and the ability to take charge of the change occurring (Anderson, Goodman, \& Schlossberg, 2012). A successful transition within college is vital to the persistence and graduation of the individual. 
Figure 3

Process of moving in, moving through, and out of transition

Moving In
i.e. leaving home and
relocating into a
residence hall

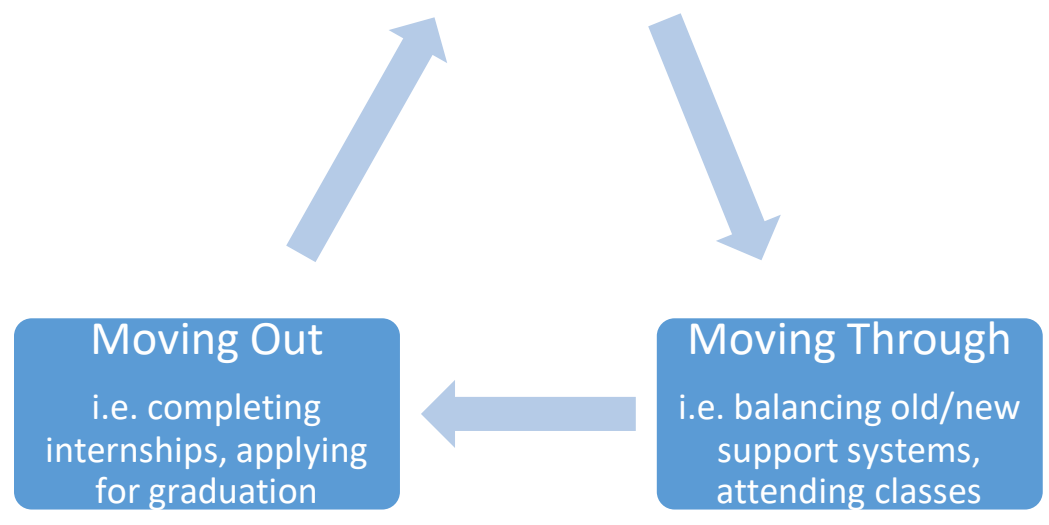

Note. By author

\section{S System}

Coping mechanisms are generally described as tools or strategies that an individual uses in response to a stressful situation (Morris et al., 2003). Schlossberg (1984) identifies four specific approaches that can assist a person adapting successfully during a period of transition: self, support, situation, and strategies. These approaches, known as the 4S system (Schlossberg, 1984), are coping mechanisms that the individual can utilize throughout their transition (Evans, 2010). By understanding the students' transitional experiences, research can guide families, communities, universities, and policymakers to assist students in successfully transitioning into college and ultimately increasing their educational 
attainment. Examining each of the four coping approaches (self, support, situation and strategies) is important as each plays a vital role in the transitional experience.

1. Self. Evans defines self as "personal characteristics such as socioeconomic status, gender, age, ethnicity, health, self-esteem, outlook, commitment, values, spirituality and resilience within self” (2010, p. 217). Expanding on this definition, Schwartz, Cote, and Arnett (2005) state that individuals must have the ability to identify attributes of their personality, which can adapt to changes within society throughout life; thus, leading to potential self-control over the personal transition. Understanding one's self-control and personality traits could influence the transition into college. For example, a first-generation student may struggle more with navigating the college process due to lack of family members having experienced college.

2. Support. Schlossberg (1984) describes support as another factor that leads to coping throughout the transition. Support refers to the level of positive interactions, feedback, and affirmation between the intimate family, friends, university personnel as well as the individual to assist in transition (Anderson, Goodman \& Schlossberg, 2012; Evans, 2010). Evans also describes the importance of stability within support to assist the individual (2010). Stability is important as it allows individuals to continue their transition knowing there will be a positive outcome (Evans, 2010). Support from one's family may be the encouragement to attend college, leave their home community, or support throughout the transition. If a student does not have positive encouragement from within their family, the likelihood of a successful transition can be diminished. 
3. Situation. Situation describes how a person evaluates the transition and determines their level of control over what is happening (Evans, 2010). Essentially, the situation is everything included within the transition, this includes but not limited to the following: triggering of the transition, timing, control over the transition, duration, previous experience with this type of stress, concurrent stress, and ability to assess of the individual's behavior (Evans, 2010). For example, if a student had prepared for college and was accepted to the school of their choice, the situation may be viewed more positively than a student who was delayed a semester or attended their third choice school. The more control a person feels over their transitional situation, the greater potential there is for a positive experience.

Factors of a situation. Schlossberg states that situation within the transition includes the trigger, timing, control, role change, duration, concurrent stress and assessment of stress. Trigger is whether the event was an anticipated transition. Timing is whether the transition was on time or off time, according to the person experiencing the transition. Control is how the individual assess the level of control within the transition. Role change is the personal position change, specifically a change of gain or loss within their community standing. Duration is the determining if the transition is permanent, temporary, or uncertain. Concurrent stress is whether other sources of stress are present. The assessment of stress is the responsibility of the person and how the individual's behavior has been affected by this transition. Overall, the situation allows for the transitional understanding and preparedness that the individual embarks on; specifically, the transition 
to college (Schlossberg, 1984). There is little research which examines the situation and transition triggers associated with students graduating high school and entering college among the AKY subpopulation.

Although, the overall research landscape is limited, there are a few studies which address various aspects of the situation within transition. Studies suggest that first generation students are less likely to persist and graduate from college (Martinez, Sher, Krull, \& Wood, 2009). However, little is known about their college experience and expectations of success (Pike \& Kuh, 2005). Pike and Kuh (2005) suggest that it is especially difficult for students to prepare for transition into college if they are also transitioning into a different racial, ethnic, or religious community. This is important as students from AKY are likely to face additional transitioning barriers as they enter their new college community. Additional research suggests that first generation students lack the realistic picture of college leading to high stress as they become overwhelmed with managing their responsibilities (Dwyer, 2001). Without strong coping methods, this lack of realistic expectations could lead to a failure within college and a new transition. As most students from AKY are first generation, they will face greater barriers due to a lack of realistic expectations within their transition to college in regard to academic, social, cultural, financial, and independence issues.

The lack of research within the realm of situation leads to a need for additional studies but also a focus on how students perceive college and what they ultimately experience. Research is also needed in regard to 
underrepresented populations and major specific transitions.

4. Strategies. Strategies are actions that lead to the ability to manage a transition (Evans, 2010). Three phases of adaptation within a transition have emerged: modify situation, control meaning, and manage stress in the aftermath (Evans, 2010). Modifying the situation could involve reevaluating the situation through a new lens, managing one's emotions to the stress, and one's flexibility within the adaptation (Chickering \& Schlossberg, 1995). Controlling the meaning of the transition occurs during the moving through phase of a transition (Evans, 2010). For example, the ability for an individual to govern their feelings of loneliness as a person adapts to a new culture at college. Managing the stress is the ability to adapt to the new stress level and create pathways to move out of the transition. In terms of transitioning to college, this could take the form of creating a schedule to manage one's time and new responsibilities.

\section{Coping Modes.}

In addition to these phases of transition, Schlossberg suggests four coping modes which can assist with adaptation as a person moves through a transition: information seeking, direct action, inhibition of action, and intrapsychic behaviors (Evans et al, 2010; Goodman, Schlossberg, \& Anderson, 2006; Schlossberg, 2012).

Strategies are valuable individually, but one must also be able to be flexible in their ability to use different approaches as needed throughout the transition (Evans et al., 2010). Strategies play an important role as transitions to college can be positive yet provide an increase in stress (Wong, 2006). Voelker (2003) suggests that first year students are at risk for new stressors (i.e., homesickness, financial 
burden, time management, and social anxiety) during their transition to college. Transitioning to college could create developmental challenges, distress, and decrease in well-being(Conley, Kirsch, Dickson \& Bryant, 2014). Developing skills which allow students to reframe the transition into a positive light (i.e., managing meaning) could be one option for coping (Folkman \& Moskowitz, 2000). These strategies as methods of adapting to transition are valuable to college students because it allows the individual to adopt modes of coping through the transition. Researchers suggest that students who become involved in activities on campus are less likely to leave campus, thus obtaining a successful transition (Kelly, et al., 2007).

Students from AKY have the potential to lack coping skills due to the other barriers they face within the $4 \mathrm{~S}$ system. For example, an AKY student is less likely to seek information because they don't know where to start to gather that information. The lack of knowledge is potentially due to the lack of familial support and knowledge of college, which leads to the inability to modify the situation within the transition. The lack of modifying the situation causes the student to stall in their process of moving through and out of the transition to college.

There are many variables and facets within one's self that influence the ability to move in, through, and out of a transition. However, self is only one aspect that leads to a successful transition. Understanding the variables which influence the "self" of Appalachian students will affect the transition of AKY students and would allow for stakeholders to develop strategies to assist in their K-12 academic preparation, college choice, major choice, and ultimately their successful transition 
into college at an urban institution while studying STEM.

Although the other coping mechanisms (self, situation, strategies, and support) influence the individual's view on their transitional situation, academia continues to lack research on the lived experience. Not understanding the lived experience of the students' transition into college can impact the retention and graduation rates within universities. "Universities must tailor retention to fit the needs of their students” (Berger \& Lyon, 2005, p. 3), but they first must understand what those needs are as experienced by the students in these situations. By gaining knowledge on the lived experience of subpopulations transitioning to college can assist the student by knowing the coping mechanisms for a smoother transition, university administration in creating programming and interventions to support the transition, as well as policy makers to create policies that support positive transition and increased graduation rates. Additionally, since subpopulations have specific experiences, understanding those experiences will be vital in developing support unique to the student subpopulation(s) instead of attempting to utilize a one size fits all approach.

Schlossberg's transitional theory although it is widely accepted within both higher education scholars and practitioners, has its limitations which need to be addressed. The theory originally developed through examination of adults transitioning into retirement is extremely complex. This is due to the acceptance of Schlossberg to criticism and the ability to expand her theory. The complexity and focus on subjective views of both the transition and adaptation leads to a lack of validated assessment tools. Schlossberg identifies many variables which can support or hinder the ability for one to adapt to the transition; however, these 
variables lack an assessment tool to validate their influence. Another limitation within this theory although it's utilized for higher education scholars examining transitions, it lacks discussion on the influence of academic preparation. Although, one could argue the lack of academic preparation could indirectly be covered by the components of "self"; it should be a stand-alone factor within self and/ or situation as research shows it's direct influence within the transition to college. The last limitation associated with Schlossberg's transitional theory is the lack of continuous flow throughout the transition. The theory while it provides analysis of moving in, moving through, and moving out; the theory only allows the researcher to take snapshots during these transitional moments. A more detailed flow with continuous evaluation throughout every stage of the transition would assist researchers in understanding the full picture of one’s transition.

\section{Conclusion}

Schlossberg's 4S system is a theoretical principle developed to assist adults in understanding their transition from career to retirement. It has been accepted as relevant by higher education scholars as a critical lens by which to review the transition into college. College transition includes moving in, through, and out of the event. The transitional experience is impacted by the person's self, situation, support, and strategies (4S system). A student's transitional experience can have a positive or negative impact on their retention within college.

There are major contributions within the literature: most notably, the understanding of transition and its importance within life. In addition, Schlossberg's transitional theory has been well established as an acceptable lens to 
examine transition (event or non-event), types of transitions, the process of moving in, through, and out of the transition. Any transition requires coping strategies analyzed through the 4S system (self, situation, support, and strategies). Furthermore, Schlossberg and Evans highlight the explanation and importance of understanding transition. Schlossberg and Tinto continue to discuss Schlossberg's theory through the lens of college transition. Schlossberg's 4 S's is a theory designed to assist individuals ability to understand their transition - researchers have referred to this as "taking stock" (Chickering \& Schlossberg, 1995, p. 49; Evans, 2010). Taking stock allows a person to evaluate their ability to move through the transition (Chickering \& Schlossberg, 1995). The ability to examine the transfer to college for students from AKY and studying STEM is important for both the ability to create more degrees within this field and to assist students in breaking the poverty cycle within their community. Examining the student experience during this transition by subpopulations allows practitioners to develop systems to support student success.

AKY and rural communities share different qualities within their social norms. Through low income, low educational attainment, and high poverty levels, the AKY community faces many challenges and barriers in closing the equity gap. Rural communities having different social norms (Stone, 2014) reinforces the need for researchers to examine the experience of different rural communities. Understanding their experiences and needs allows practitioners to better support subpopulations and their specific needs. Community cultural wealth (cultural capital, linguistic capital, resistant capital, navigational capital, social capital, familial capital and aspirational capital) contribute to the communities perceived 
level of support for higher education (Yosso, 2005). Community cultural wealth discusses the aspects which can positively or negatively affect a student's likelihood of success. Universities are in need of reviewing their policies and interventions to ensure that all students, including AKY students, have access to cultural capital wealth. Simple evaluations of family weekends and providing scholarships to parents who may not be able to afford to travel, or sponsoring buses for AKY students to visit campus through their high school could potentially impact the community cultural wealth of this subpopulation. Although there is research on rural students attending college, there is no research on rural students who attend an urban institution studying a STEM field. STEM education is a rigorous pathway within college, with the potential to be one of the highest paid and largest growing fields within the United States. The need for STEM majors and degrees is well researched; in addition to factors that lead to success within STEM. There has been a record number of STEM majors within higher education, however not enough to meet market demands. Therefore, it is vital for research to examine AKY rural students and their transition into an urban STEM major.

Transitioning to college can be one of the most influential changes in one's life. A successful transition into college and the first year is a major indicator of persistence and success towards degree completion. Transitioning to college is a stressful time, specifically as students focus on academic and social transitioning. Tinto (1993) stated that the successful transition to college is a major factor in predicting the success of a student.

There continues to be a need for research on rural AKY students. The gap within research lacks a lens on specific academic studies, oppressed 
subpopulations, and its effect on the transition into college. Such research would help shed further light on the impact of leaving a homogenous community, entering an urban community, adjusting to a new city and social norms within higher education, adapting to academic expectations, and constant attack on one’s self esteem.

Additional gaps within the rural communities and student success literature include a lack of focus on specific academic units. Research states that rural communities have unique attributes, specifically across other rural communities (Gibbs,1989). Understanding these communities and the effect of transitions among the population is important to facilitating improvement within the community.

Throughout Schlossberg's transitional theory research, one aspect remained undiscussed: academic preparation. Academic preparation is a well-known success factor among STEM majors in college. Academic preparation is a portion of self, which needs to be examined through a transitional lens. Knowing that academic preparation within K-12 education and the importance to strong academic programming leads to an increase chance of persistence within higher education, one must examine the transition from high school to college. Academic preparation is extremely valuable in transitioning to college as it has the potential to have other impacts on academic expectations and social norms of academics. STEM academics typically recruit top talent into the major; this could lead to an entirely new community, and additional adjustment is needed for the transition. For example, when 500 top students from high school join a cohort, they must adjust to the likelihood they will no longer be the top student. This change has the potential 
to affect a person's transition and could lead to persistence issues.

The need for literature examining the transitional experience of AKY students attending an urban institution and studying STEM adds many benefits to research, particularly giving a voice to those students. AKY students face additional barriers during their transition due to the intersectionality they hold as individuals. Those barriers have the potential to negatively impact their transitional experience into college, thus leading to lower retention and graduation rates; this becomes even more persistent when another barrier of rigorous academic discipline is added, such as STEM. In addition, this research can allow for the opportunity to understand factors that influence a positive transition into college as well as providing insight to practitioners on avenues to assist a minority population transition. This research can also encourage other students from Appalachia to attend college at an urban institution and study STEM. Additionally, this research can add to the body of knowledge within the following scholarly realms: college transition, STEM education, rural communities, and qualitative data with STEM. 


\section{CHAPTER 3: METHODS}

This chapter presents the research questions, an overview of a pilot study (which utilized the research questions), the methodology approach, and limitations. This qualitative study approaches the research through a case study lens, using both interviews and focus groups as the data collection method.

The central question of this study is: how do rural students studying in a STEM field experience their transition (situation, self, support, and strategies) in college at an urban university? This study utilized a case study approach in exploring the experience of AKY students. This approach allows for the experience to be shared in a meaningful way as well as examine the meaning within the transition (Creswell \& Poth, 2018). This approach best suits this qualitative study as we examine the lived experience of how students from Appalachia KY transition into studying STEM at an urban institution and strive to ensure that the individual shares their experience. By utilizing Schlossberg's transitional theory, this allows the researcher to make meaning of the transition while also examining the application of this theory within higher education for vulnerable and marginal students. It is also important to note that individuals from Appalachia may hesitate to share their experience due to many documentaries and research studies which suggest a bias lens when examining the lived experiences of rural America. By utilization of a phenomenological approach, individuals would be able to share their experiences drawing from their prior experiences. 


\section{Research Questions}

To answer the central research question the researcher created the following questions:

Q1: What individual factors influence the transitional experience?

Q2: What factors of the individual's support system (family, hometown and university) influenced the transitional experience?

Q3: What factors within an individual's situation influenced the transitional experience?

Q4: What factors within the individual's strategies influenced the transitional experience?

\section{Rationale for qualitative methods}

Qualitative research was selected for the following reasons: depth over breadth of data, focus on the lived experience, understanding of the transition, and expressing the participant’s story (Creswell \& Poth, 2018). Quantitative data allows for a surface level analysis of a situation, however qualitative research allows for the researcher to gain an in depth understanding of the transitional process. Qualitative research allows the participants to share their lived experience, reflecting on their feelings, strategies, and responses throughout the process. Additionally, qualitative research encourages the researcher to understand the transition and to share the interviewee's story.

\section{Approach}

The researcher utilized a case study approach to gain a deeper understanding of how AKY students transitioned to college, while studying STEM at an urban institution. Creswell \& Poth (2018) state that a case study is most appropriate when an insightful understanding of experiences is needed within a subpopulation. As a subpopulation, 
understanding AKY students lived experiences allows university faculty and staff to enhance the transitional experience and potentially increase the likelihood of success within the university. The AKY subpopulation studying STEM at an urban institution has not been researched; thus, the research body of knowledge lacks students' shared experience transitioning to college. By providing an outlet to share their experience, practitioners can begin to create policies and interventions to support these students' transition into college. According to Schlossberg (1984) adaptation to transition is highly individualized, as is the experience of the transition. Because of this theoretical belief, it would be important to engage each individual, and create an environment where they would be allowed to reflect and share their experience.

A case study allows an examination of the transition through the voice of students who are living the experience and recommends collecting data through an interview format (Creswell \& Poth, 2018). Case studies provide an in-depth and detailed discussion regarding a person’s experience over a specific event (Creswell \& Poth, 2018). Case studies could examine an individual, group, situation or action; thus, utilizing the case study approach ensures that participants are able to discuss their transition while also reflecting on their intersecting identities. Case study was selected to allow individuals to share their experience in transitioning to college and allowing students to engage in an indepth conversation while allowing for inspection of their adaptation. This approach was vital with this specific subpopulation due to the nature of research and documentaries focusing on the negative aspects of this community. By providing a voice to students from AKY who have transitioned, researchers can understand their experience through their lens. This study is important as current research has yet to capture the transitional experience of students from AKY while studying STEM at an urban institution. 
Therefore, researchers, practitioners, and policy makers currently lack guidance in enhancing the transitional experience of AKY students.

\section{Positionality}

Having spent four years throughout my college experience living and studying in the Appalachian region of Kentucky, I have a personal tie to the region. I selected my alma mater for the same reason as many of these participants, the scholarship and sense of belonging. It is there that I met my best friends, figured out who I am as a person, and where my heart still feels at home. I spent my time learning about the world not only through my college instructors but also the people within this rural community. I hiked the Appalachian Trail, attended community festivals, and met individuals who were affected by the elimination of coal jobs in their region. I met individuals who were first generation college students and were looking for a way out of poverty. I saw firsthand the good nature of this community but also the lack of economic development due to the lack of education of its community members. This is the region that my niece and nephew call home, as well as where my sister and brother in law have chosen to raise their children. My passion for this research derives from the possibility of bringing true opportunity for economic and educational growth to the region.

I remember my experience transitioning from an urban childhood to a rural community and it was a true learning experience, especially within the culture of the community. Within the Appalachian community, everyone knows everyone and there is pride both in family as well as the community. Coming from a "big city" you were accepted but at arm's length, their loyalty lay with their family and the town in which they grew up in. You were expected to know cultural norms which most individuals were exposed to from birth and yet were completely new to me. I still remember 
traveling home with a friend to meet their family. Although they were welcoming with open arms, they also knew based simply on the way I spoke that I wasn't from their county. In fact, I found out quickly that county pride was very important. The different counties within eastern Kentucky had specific norms and stereotypes. I remember my friends had assumed that I had never seen a cow in real life or that I lacked the ability to drive a four-wheeler. I personally struggled with trying to fit into a community I loved so much, while at the same time holding on to values that made me who I was at the core. I think that most students from rural Kentucky will struggle with the diversity of an urban school, the world views of their new-found peers, as well as endless opportunities to become distracted. I believe this could be a struggle as many Appalachian students have grown up in a homogenous community with mostly white families. As many participants shared within this study coming to college was the first time, they met someone who wasn't white, wasn't Christian and/ or didn't have conservative values. Participants within this study shared how they had been told that the "big city” was filled with crime, danger, and highways. It was a fast-moving town with fast moving people. One of my favorite things about eastern Kentucky (besides the beautiful mountains and nature) is how the time seems to move a little slower; the days seem to last a little longer and the world is a little more peaceful. Most importantly, I believe students from a rural community will struggle with the lack of community support or familial support within close proximity. However, given the opportunity, I believe AKY students can have a positive impact on the culture within an urban institution.

There are many things that I will gain out of this research. I am gaining an opportunity to learn more and impact a community I care for deeply. I am also securing 
the opportunity to develop best practices for practitioners to assist such a vital population. There is also the opportunity to contribute to the body of knowledge within college student development.

\section{Critical Theory}

Critical theory was developed to provide a voice and pathway for change of oppressed and underrepresented populations (Creswell \& Poth, 2018). Creswell argues that this theory is utilized mostly for populations such as female and minority populations, allowing individuals to share their experience who typically are not given a voice. Appalachian Kentucky students who are striving to obtain a higher education should be categorized as an oppressed population. There are two main reasons why AKY students should be categorized as underrepresented population: (a) the reality of potential oppression exists within the Appalachian community through their social, political, and cultural norms; and (b) there is direct pressure from family and indirect pressure from community members to stay within the community and obtain jobs that are currently available within the community, instead of seeking a higher education degree.

This research is designed to share the experience of transition from the lens of the participants and analyzed through Schlossberg's transitional theory. Moreover, this process adopts a critical stance, which focuses on subjective opinions within social, political, and cultural context.

\section{Study Methods}

This study was completed as a qualitative study, utilizing a case study approach. Additionally, this study obtained Internal Review Board (IRB) approval, which is important to provide opportunities for publications and articles surrounding the research obtained, in addition to sharing the voice of students among this population. During the 
IRB submission process, the researcher reviewed and expanded the interview questions that occurred to capture the "transitional” experience through the lens of Schlossberg's transitional theory. The interview protocol was developed to ensure both reliability and validity of the study. The interview questions were developed to analyze coping mechanisms throughout the transitional experience through Schlossberg's transitional lens; a total of 19 questions were developed for one-on-one interviews and 13 questions for the focus group (appendix B \& C). The following section describes the research methods approach, a qualitative approach lens, data collection, and ethical considerations all used in the proposed research.

\section{Purposeful Sampling}

Criterion sample was utilized to select potential participants to ensure the specific population was included within the recruitment process. Criterion sampling is an approach to ensure all participants meet a common set of criteria to be included in the study (Creswell \& Poth, 2018). Criterion sampling is important for a case study as it ensures the researcher is sampling from the appropriate population, whose voices are currently not captured, and allows for quality assurance within a study (Creswell \& Poth, 2018). With IRB approval, a list of students studying STEM with a home zip code located in Appalachia Kentucky was obtained from the university technology and data office utilizing the following inclusion criteria:

1. Currently enrolled undergraduate student in a four-year bachelor's program;

2. Majoring in science, technology, engineering or math; and,

3. Home zip code located in the KY Appalachian region according to the Appalachia Region Commission. 
Appalachia region zip codes were determined by counties included in the Appalachian Regional Commission database. The researcher utilized the purposeful sampling and building trust through professionalism to enhance the trustworthiness of participants. To ensure that only individuals who met the criteria for this study were included, purposeful sampling was utilized to recruit participants. 127 participants were identified and a total of 14 participants agreed to be interviewed either through a one-on-one interviews or attended a focus group.

Figure 4

\section{Appalachian Regional Commission identified AKY counties}

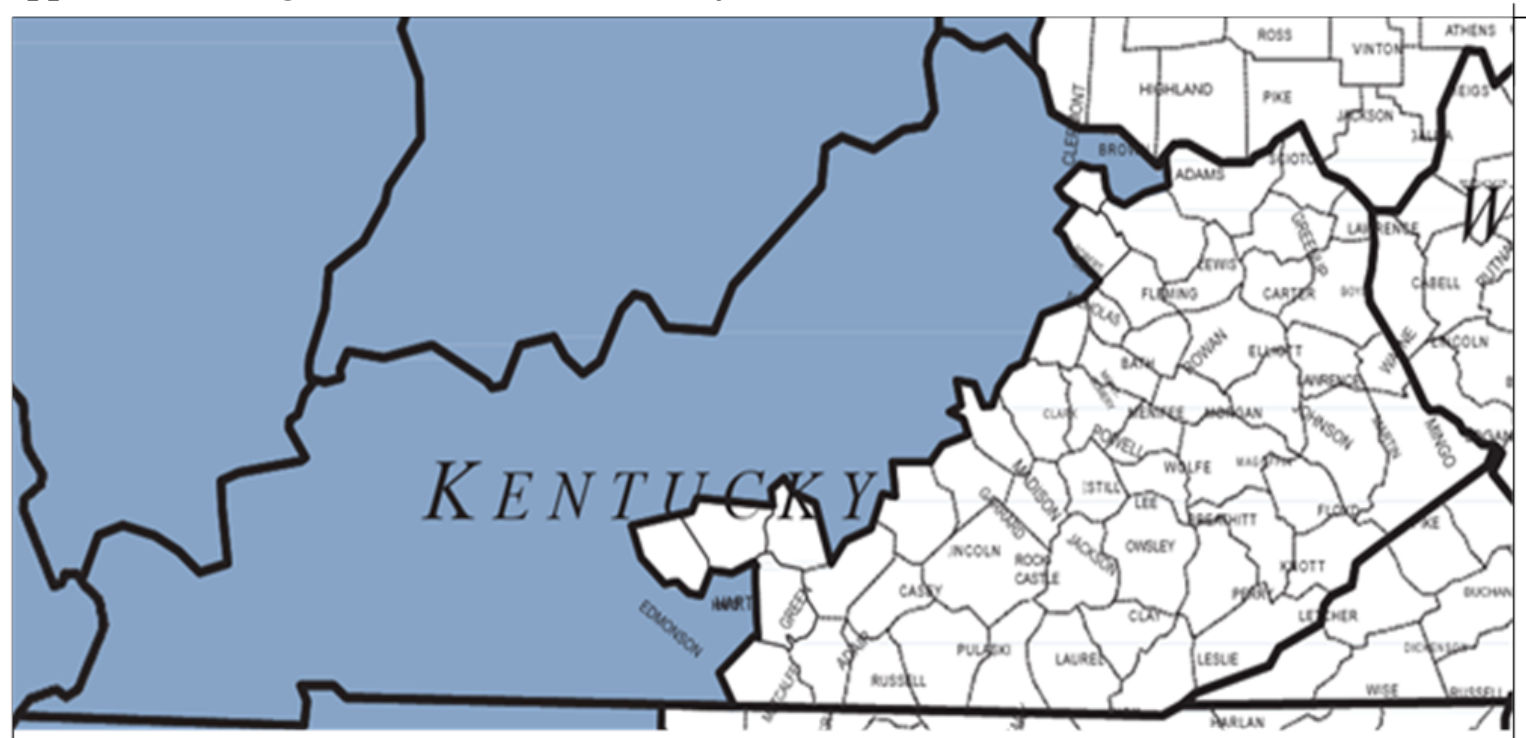

Kentucky Appalachian Counties: Adair, Bath, Bell, Boyd, Breathitt, Carter, Casey, Clark, Clay, Clinton, Cumberland, Edmonson, Elliott, Estill, Fleming, Floyd, Garrard, Green, Greenup, Harlan, Hart, Jackson, Johnson, Knott, Knox, Laurel, Lawrence, Lee, Leslie, Letcher, Lewis, Lincoln, McCreary, Madison, Magoffin, Martin, Menifee, Metcalfe, Monroe, Montgomery, Morgan, Nicholas, Owsley, Perry, Pike, Powell, Pulaski, Robertson, Rockcastle, Rowan, Russell, Wayne, Whitley, and Wolfe

Note. AKY (Appalachian Kentucky). By author

\section{Site Description}

A Midwest university in an urban setting within Kentucky was selected for this study. It is the only urban university offering STEM education within the Commonwealth of Kentucky. The second reason for the selection is that the University is one of only four STEM degree granting colleges in the Commonwealth of Kentucky. 


\section{Pilot study}

A pilot study was conducted in 2019. The data collection and analysis occurred during a semester in which the researcher was enrolled in a qualitative research course. This pilot study obtained Internal Review Board (IRB) approval, which is important to provide opportunities for publications and articles surrounding the research obtained, in addition to sharing the voice of students among this population. Six participants partook in the study. Three participants agreed to participate in one-on-one interviews. The remaining three participated in a small focus group. The one-on-one interviews were asked 19 questions designed to provoke thought and discussion of the individuals transition into college while embarking on the rigor of studying STEM within a new community of an urban institution. The focus group included questions designed to engage students to discuss their transition and adaptation of their transition to college. Both the oneon-one and focus group interview protocols can be found in appendices B \& C. Data from the interviews and the focus groups were recorded, transcribed by NVivo, and sent to participants for confirmation.

A formalized approach of first cycle, transitional cycle, and second cycle as introduced by Miles, Huberman \& Saldana (2014) was conducted to build credibility into the findings. During the first cycle of coding, methods included causation coding, descriptive coding, and elaborative coding. 
Table 2

First cycle coding definition

\begin{tabular}{|l|l|}
\hline First Cycle Coding Type & Definition \\
\hline Causation Coding & $\begin{array}{l}\text { Analyze the causality by identifying causes, } \\
\text { outcomes, and links between them }\end{array}$ \\
\hline Descriptive Coding & Describe the topic of data with descriptive nouns \\
\hline Elaborative Coding & $\begin{array}{l}\text { Develop codes to refine theoretical constructs } \\
\text { emerged from previous research or investigations }\end{array}$ \\
\hline
\end{tabular}

Note. By author

These semi-structured interviews and focus groups provide a cross case analysis highlighting four major themes: 1) social support system with deeper relationships; 2) lack of academic preparation; 3) university support by assisting an individual; and 4) individual adaptation capabilities. Preliminary findings suggested the importance of finding support and identified other coping mechanisms. These findings reinforced the need to continue researching this topic.

\section{Recruitment of Participants}

Potential participants received an introductory email requesting participation in either a face-to-face interview or focus group (see appendix A). A total of four recruitment emails were sent to potential participants. A date and time were established matching the interviewee and interviewer's availability; the focus group was pre-set with a date and time. One-on-one interviewees were offered to meet in the researcher's office or another private location more comfortable for the participant. The focus group was held in a meeting room on the university campus.

\section{Number of Participants}

A total of 14 participants were included in this study; eleven face to face interviewees and three attended the small focus group. Three face to face and three within the focus group were participants utilized within the pilot study. The additional 
eight participants interviewed through face to face were added for the purpose of this study. These participants, through interviews and focus groups allowed for the data collection, understanding, and sharing of ones' transitional experience as students from AKY, studying STEM at an urban institution. Below is a brief description of each participant. Table 3 provides a meta view of participants and background information.

Table 3

Demographics \& background

\begin{tabular}{|c|c|c|c|c|c|c|c|c|}
\hline Participant $\mathrm{C}$ & Gender & Pseudonym name & Ethnicity & Class Standing & Financial Aid & Major & \multicolumn{2}{|c|}{ First Generation Living Location } \\
\hline $1 \mathrm{~F}$ & Female & Mary & Indian & Senior & Scholarship & Biology & No & Off Campus \\
\hline $2 \mathrm{~F}$ & Female & Kayla & White & Junior & Scholarship & Biology & No & Off Campus \\
\hline $3 \mid 1$ & Male & Jack & White & Junior & Loans & Engineering & Yes & Off Campus \\
\hline $4 \mathrm{~F}$ & Female & Abigail & White & Freshmen & Scholarship/Loans & Biology & No & On Campus \\
\hline $5 \mathrm{~F}$ & Female & Sarah & White & Junior & Scholarship/Loans & Computer Science & No & Off Campus \\
\hline $6 \mid 1$ & Male & James & White & Sophomore & Scholarship & Chemistry & Yes & Off Campus \\
\hline $7 \mid$ & Male & Michael & White & Sophomore & Scholarship & Chemistry & No & Off Campus \\
\hline $8 \mid 1$ & Male & Sam & White & Sophomore & Scholarship & Engineering & no & Off Campus \\
\hline $9 \mathrm{~F}$ & Female & Jessica & Two or more & Sophomore & Scholarship & Engineering & Yes & Off Campus \\
\hline $10 \mathrm{~F}$ & Female & Maria & White & Freshmen & Scholarship/Loans & Engineering & Yes & On Campus \\
\hline $11 \mathrm{~F}$ & Female & Georgia & White & Freshmen & Scholarship & Engineering & No & On Campus \\
\hline $12 \mid$ & Male & John & White & Junior & Loans & Engineering & No & Off Campus \\
\hline $13 \mathrm{~F}$ & Female & Denise & White & Senior & Scholarship & Chemistry & Yes & Off Campus \\
\hline $14 \mathrm{~F}$ & Female & Taylor & White & Freshmen & Scholarship & Chemistry & No & On Campus \\
\hline
\end{tabular}

Note. By author

\section{Participant Information Participant 1 (Mary)}

Mary moved to eastern KY around the age of two. Her parents did not hold college degrees from the United States but owned and operated a business. Her college offered her the best scholarship package and it was where she felt most comfortable with the university after a campus visit. She also followed her brother to the same university, who she frequently claims was a major factor in her success majoring in STEM. The diversity of the student population allowed her to connect with her Indian heritage, which 
assisted in her transition. However, the academic expectations and lack of academic preparation was stressful at times during her transition into college.

\section{Participant 2 (Kayla)}

Kayla is a female who moved to Appalachia at a young age. She was top of her class in academics and involved in academic programs such as Science Olympiad (a STEM focused student organization) and Beta Club (a leadership and service organization). Her transition was one of excitement as she was looking forward to a new start, making new connections, and redefining herself as a person. Her home community lacked racial and religious diversity. She was nervous about the highway, as there is only one main road in her town; however, she was ready to conquer these barriers.

\section{Participant 3 (Jack)}

Jack is from eastern Kentucky where he grew up his entire life. In high school, he did move to another town in eastern Kentucky for the educational opportunities available at the high school and to avoid the drugs that were moving into his hometown. Growing up on a farm was extremely enjoyable, and he still remembers living on the same 100+ acre farm as his grandparents, raising cattle and chickens. Jack applied to universities across the US such as MIT but decided to attend this institution because he didn't want to be that far from home.

\section{Participant 4 (Abigail)}

Abigail was originally born in Ohio, because there were no hospitals in her AKY hometown. The family home lacked internet until high school and still utilizes well water. She spent a lot of time outside and grew an appreciation for the environment. Living next door to their grandparents allowed for a strong extended family relationship. High school was easy as many days were spent playing cards. And although the 
transition to college was difficult, she firmly believes that her school prepared her academically for success.

\section{Participant 5 (Sarah)}

Having two parents who worked for at a university in her hometown, Sarah knew going to college was always an expectation. Growing up in the most eastern county in Kentucky, with only a Wal-Mart and a few restaurants, didn’t leave Sarah with much exposure to big city culture. Having STEM run in her veins, it was easy to see how her family life incorporated her love for STEM education through outdoor play and science experiments. Her grandfather was an Engineer, leading to early exposure to the STEM profession. The opportunity to utilize the Kentucky Educational Excellence Scholarship (KEES) funds and university scholarships allowed her to make the decision to attend the university of her choice.

\section{Participant 6 (James)}

James grew up in a family with little money, but that didn’t stop him from dreaming of getting out of Kentucky to see the world and explore various cultures. As an introvert, high school was not pleasant as he wanted to spend more time being on the academic team than hanging out with his classmates. Having an older sister who went to medical school allowed him to see a pathway out of Eastern Kentucky. Out of his class of 120 seniors, he remembers only about 20 going on to college. Access to the governor scholars program allowed him to truly focus on expanding his horizon in terms of access to college and various majors.

\section{Participant 7 (Michael)}

Michael had a father in the military, whose service caused Michael to split time growing up between his grandparents and father’s houses. Having little to no interactions 
with his mother, he describes her as a bad influence. His high school continuously scored below the standards set forth by the state of Kentucky and he can still recall the posters stating what those standards should be within each grade. His high school career consisted of going to school at 8am - 3pm and then working until midnight, which became a necessity to assist in providing food for the family. His love for STEM developed when he was six years old while his dad was enrolled in a chemistry college course.

\section{Participant 8 (Sam)}

Growing up in north eastern Kentucky provided access to a few restaurants, a gas station and a Wal-Mart. There was very little to worry about as it was a fairly safe town, and everyone knew everyone. During high school Sam took every AP course his high school provided and was heavily involved in sports as well as academic teams. During high school he initiated an effort to bring dual credit into the school for students to develop a competitive edge for college. Although the culture within the community was not a college going culture, that was the focus within his high school. Since Sam had always been interested in science and math, STEM seemed a good fit in college.

\section{Participant 9 (Jessica)}

Jessica grew up in Liberty Kentucky, with six brothers and sisters. She never really felt she fit in; however she got involved in beta club and Upward Bound (precollege organization focused on academic success) which became gateways into deciding to come to college. After meeting with a guidance counselor, she decided to attend college. The first semester she joined a student organization, finding the familial support needed, assisting in her ability to become successful in her transition. In addition, her first semester was extremely easy which allowed her to develop a support 
system and focus on her academics the next semester as her courses became more difficult.

\section{Participant 10 (Maria)}

Growing up in Ashland Kentucky played a unique role for Maria wanting to study STEM. Ashland Oil is located within the city so there are hopes of good jobs for educated individuals. Maria was actively involved in high school: golf, beta club, academic teams, and was student body president. Having a friend who attended the same university and studied engineering, she had inside knowledge on bioengineering and how it could lead to a pathway to attend medical school. The decision to attend her selected university was solidified financially through scholarships provided by the university. Support was provided by those who had attended college, parents, siblings and guidance counselors. The anticipation for college was strong, both to be pushed by the academic intensity and the peer intelligential capacity.

\section{Participant 11 (Georgia)}

Georgia grew up in Adair County, a typical rural community with two stoplights and a Wal-Mart. This town has been hit hard by the opioid crisis. In fact, Georgia mentions friends being arrested for meth possession and others who have overdosed from opioid use. As a self-described loaner kid, she did not have many friends growing up. Family were nearby with grandparents, aunts, and uncles living within the same community. Georgia's high school involvement included the pep club. Her father was a biology professor, so STEM was “in her blood”. Moving to an urban institution was a major culture shock, with the increase in traffic and diversity. Scholarships and support programs such as Girls Education and Mentoring Service (GEMS) influenced her 
decision to attend the university. Georgia identified studying was a major factor needed to become successful throughout her college experience.

\section{Participant 12 (John)}

John attended the focus group. He spent time growing up in Lexington as well as in Appalachia, KY. His family moved frequently due to job and housing opportunities. The transition seemed to not be as complicated for John as he began at a community college before transitioning to his selected university. John relies on his parents for support through his transition and success within college.

\section{Participant 13 (Denise)}

Denise grew up east of Lexington and does not consider her home town to be in Appalachia, “ the true eastern Kentucky.” Her selection of college was based on feeling comfortable on campus, a smaller community and more affordable housing than other universities. However, her family expressed concerns about the big dangerous city. Denise states the importance of her mother playing a major role in assisting Denise in applying and attending college. She also participated in Governor Scholars Program, a summer enrichment program which exposes high school students to various colleges across the state during their senior year in high school.

\section{Participant 14 (Taylor)}

Taylor grew up in a holler of Appalachia Kentucky with a “classic Appalachian experience.” She was proud of both her upbringing and success within college. Her family expectations were if she went to college then she would be responsible for the finances. She chose to attend this university because of the scholarships which were offered to her. Her transition was tough, she stated "no matter how ready you are for the 
transition, it’s still a big transition." The stressors include moving to a large city, classes and being so far from home.

\section{Data Collection}

All interviews occurred face-to-face in the researcher's office and the focus group took place in a private study space on campus. A tape recorder was utilized to capture all interview sessions. All recorded interviews were uploaded into NVivo and transcribed for analysis.

One-on-one and focus group interview questions were designed to capture the transitional experience of students moving from rural Kentucky to an urban institution while studying a STEM major (see appendices B \& C). The questions between the oneon-one interviews and the focus group vary as the focus group was focused on support strategies within the transition. This study utilized semi-structured interviews and focus groups, with interviews lasting on average 60 minutes. Interview questions began broadly with an overall question about the transitional experience. Additional questions were then asked based on the 4S's within Schlossberg's transitional theory.

\section{Face to face interviews}

11 face to face interviews were conducted within the researchers office (participants were given the option to meet at a different location, but they all declined). The questions designed for the face-to-face interviews included 19 total questions, beginning broadly with the overall experience of transitioning to specific questions focused on the self, situation, strategies, and support throughout the transitional period of entering college. Face to face interviews in a semi-structured model allowed the researcher to adapt follow up questions and probing questions as the direction changed or the information shared by the participants needed additional probing. For example, Jack 
shared the struggles of feeling as if he wasn't as smart as the other students within his major. The semi-structured approach allowed the researcher to ask Jack for more details and to describe how that felt as well as what he did to "overcome” the feelings.

Participants were informed of the researchers' interest in this study and the ability to end the interview at any point.

\section{Focus group}

One focus group was conducted for this study. The email solicitation for participants in this study provided options for either face to face or a focus group. A majority of students responded that they were interested in one-on-one interviews over the focus group interview. This is abnormal, as this subpopulation is highly connected to community and thus one potentially could assume that individuals would prefer to gather in groups as oppose to one-on-one interviews. As was shared by multiple individuals during this study, being at a urban institution creates a feeling of needing to hide where they are from, their beliefs and at times, even their accents; as they fear being judged negatively.

Questions within the focus group centered on the overall transition to college, as well as situation, and support systems within their transition. Knowing that time was limited, the researcher decided to focus their situation of selecting the university, their major, and support systems utilized to adapt as they transitioned to college. University, major selection, and support systems seemed most important as they have the potential to impact both the recruitment into STEM and retention methods for practitioners. Additionally, situation and support were highlighted factors during the pilot study. 


\section{Ethical Issues}

Creswell and Poth (2018) state that ethical considerations should be considered throughout the study, from design to conclusion. In working with humans as subjects, there are a variety of emotional, physical, and psychological issues that could be imparted during interviews. Other ethical considerations involve the rapport and trust that the interviewer must establish with their participants in the study. The researcher needs to be careful about the type and depth of personal information provided to the interviewees to ensure bias of researcher does not occur. Another ethical consideration is the treatment of the findings and disclosure of information. It is vital that each interviewee's story be shared through their voice and not through the researcher's assumptions or lens. To ensure that the voice is true to the interviewees, participants had an opportunity to review their transcript and provide corrections/ feedback where needed.

\section{Trustworthiness}

To build valid results, a researcher must establish their trustworthiness with subjects through credibility, validity, and authenticity (Miles, Huberman \& Saldana, 2014). These aspects of trustworthiness can be developed through multiple avenues. This researcher has focused on the following approaches to gain participant trustworthiness: confirmability, by ensuring participants’ responses are expressed correctly; triangulation; and dependability, by ensuring the study can be easily replicated as described by Huberman, Miles and Saldana (2014). To ensure that the context was rich and made sense within the data collection, the researcher followed strict interview protocols, recorded the data collection, and reviewed the transcripts on multiple occasions. Triangulation occurred in multiple ways; first, the researcher recorded all interviews and made memoing notes. Second, all transcripts and quotes were sent to 
participants for review and corrections made as requested by the participant. The researcher stayed in close contact with her dissertation chairperson and methodology committee member to ensure data collection, analysis, and writing correctly represents the data. Lastly, the data has been reviewed by original participants; this was obtained by bracketing (removing the researchers bias and current knowledge of the research) and ensuring the participants had access to both their transcripts as well as any direct quotes used in the research paper.

\section{Transcript Review}

The focus group and all interviews were recorded and transcribed by NVivo software system. Transcripts were then reviewed, line by line, for accuracy. Each participant was provided with a copy of their transcript and the study for feedback and/or corrections. Engaging the participants in reviewing of their own experience and words enhanced the data credibility by ensuring that their voices are being shared according to their lived experience.

All transcripts were uploaded into a qualitative NVivo software system on a university computer, which is password protected. Through these steps in uploading the transcripts, review, and write up, the researcher established trustworthiness.

\section{Coding reliability}

Ensuring there is no shift or change in voices of participants among codes, Creswell \& Poth (2018) suggest that researchers continue to review codes and data to ensure they are matching the participants' meaning. Transcripts were read a total of four times. The first and second reviews were to develop an understanding of the participants, the third reading allowed for the development of codes within the data, and the fourth 
involved categorizing the coded information. The codes and categories were placed into a matrix of the transitional process (moving in, moving through, and moving out) to determine what themes emerge and if/how categories fit within the 4S system (self, support, situation, and strategies) of Schlossberg's transitional theory and to what extent each "S" is utilized within the transition.

\section{Limitations}

There are a few potential limitations associated with this study: theoretical framework, university representation, and Appalachian representation.

The theoretical framework limitations include the process of snapshots during the transition instead of continuous flow and observations. Schlossberg's theory directs the reader to assume that transitioning follows the flows of moving in, moving through, and moving out; however there lacks the process of if an individual reverts back to a specific phase within the transition. Transitioning is highly individualized process, however this theory lacks any suggestion of timeframes which should be spent within the transitional phase. Additionally, due to the continuous expansion of the theory there lacks an instrument for measurement of reliability. Although Schlossberg's theory is accepted across various disciplines because of its generalizable conceptual approach, however this leaves specific factors which affect a transition into college out of the equation. A specific example of this is academic preparation. Research indicates that academic

preparation plays a role within a students ability to retain at a university, yet it's left out of Schlossberg's transitional theory.

Students were interviewed from one university thus, only similar urban universities, would be able to share similar factors of transitional barriers or provide the 
opportunity to highlight additional barriers within the transition. AKY students attending a different type of university may face similar or different barriers.

This study examined the transitional experience of students from rural Kentucky Appalachia but does not include the other Appalachian regions. This may lead to nongeneralization as students from other Appalachian states may have different community identities or struggles, which would lead to different coping mechanisms when transitioning to college.

\section{Conclusion}

This chapter discussed the methods utilized in this qualitative study. Purposeful sampling and criterion sampling were used to ensure the subpopulations intended for the study were included. Criterion sampling was used to identify individuals who met the following requirements: enrolled student, studying STEM, and a home zip code within the Appalachia KY region. The sub categories were generalized into categories and then themes developed. Themes will be reviewed within the transitional process (moving in, moving through, and moving out) as well as the $4 \mathrm{~S}$ system. 


\section{CHAPER 4: DATA ANALYSIS}

This chapter examines the analysis of the data. A formalized approach of first cycle, transitional cycle, and second cycle as introduced by Miles, Huberman \& Saldana (2014) was conducted to build credibility within the findings. During the first cycle of coding methods included the following: causation coding, descriptive coding, elaborative coding and in vivo coding. Second round coding entailed pattern coding where first round sub categories were examined into categories. Those categories were then examined through protocol process examining the set themes: self, situation, strategies, and support within Schlossberg's theory. These changes added depth, trustworthiness, and validity to the current study. Purposeful sampling and building trust through professionalism occurred to enhance the trustworthiness of the researcher to the participants. Second round categories were then examined through a protocol process to examine the set themes: self, situation, strategies, and support within Schlossberg's theory. The Schlossberg transitional lens added depth, trustworthiness, and validity to the current study. Throughout this section the researcher described the analysis for coding cycle 1 , transitional coding, and cycle 2 coding. The data was moved from sub categories, to categories, and into themes utilizing the $4 \mathrm{~S}$ system (self, support, situation, and strategy) within the framework. First cycle coding utilizes the $4 \mathrm{~S}$ system as a framework for coding, the second cycle coding utilizes those themes through the lens of the transitional phases (moving in, moving through and moving out). 
A case study was utilized in this study as it follows a set of acceptable procedures in analyzing collected data; this increases the likelihood of valid and useable information (Creswell \& Poth, 2018). Creswell \& Poth (2018) argue that researchers need to find statements about how individuals experience a situation, then grouping into meaningful units.

\section{Analysis}

An in-depth analysis of the data was conducted through first cycle, transitional, and second cycle coding. First cycle of coding was conducted using emotional, In vivo and concept codes analyzing through a chucking review process. Chucking is a process in which similar words and phrases are placed together to develop a sub category. Emotional coding includes experiences or emotions recalled by the participants (Miles, Huberman, \& Saldana, 2014). This coding is important as this research strives to capture the lived experience of AKY students transitioning to college, studying STEM at an urban institution. Emotional coding includes phrases such as Georgia describing her feelings of loneliness "I felt as if I wasn't being welcome here (the university) but also not really being welcomed at home”. Emotional themes throughout the interview included: loneliness, selfdoubt, determination, focus, adaptation, sadness, struggle, and excitement. Many students described a mix of feelings throughout the transition as being both scared and excited. Maria described moments during her transition where she took time to self-reflect and questioned the ability to succeed "I took a step back and I kind of questioned am I prepared for this? Is this the right fit, am I qualified to be in this 
major? I struggled with this for a little bit and talked to my parents”. Emotional coding allows for the researcher to capture the feelings associated with the lived experience.

In Vivo coding describes coding in the words, terms, and phrases of the participants such as "slap in the face” as stated by participant 1 , 5, and 11 . In vivo coding allows the researcher to capture the experience through the lens of the participants. Mary describes her transition as follows: "I was so scared because I knew that I was underprepared, but college was a slap in the face”. Sarah discusses her experience of transitioning and her worries "being from Eastern Kentucky to worry that people will stereotype you and might look down upon you from where you're from”.

Lastly, within the first cycle coding concept coding was utilized to allow the researcher to gather data surrounding a specific concept. This process allows the researcher to specifically examine an event of participants. This coding process is important as the research question is designed to examine the transitional experience of a specific subpopulations. Sarah describes the adaptation or moving out of the transition as “I feel totally at home and I don't feel weird or out of place here. I feel at home here”. Through concept coding the researcher had the opportunity to specifically ask questions throughout the transitional experience. These first cycle sub categories can be found in appendix E. Self-coding mapping allows for a visual diagram on cycle 1 and transitional coding. These sub categories and categories have the ability to influence the ability for participants to adapt within their transition to college while studying STEM at an urban institution.

The researcher developed sub category mapping during the transitional 
process, between the first and second cycle. The sub category mapping in the below maps illustrates the transitional process of sub categories being clumped together into categories within Schlossberg's 4S system: sub categories identified through first cycle coding, sub categories were identified as having a positive and/ or negative influence on the transition, and which phases in the transition the sub categories appear (moving in, moving through, and moving out). The coding maps include: self, support, situation, and strategies. 


\section{First Cycle Coding}

Figure 5

Thematic map showing "Self" as the first major theme
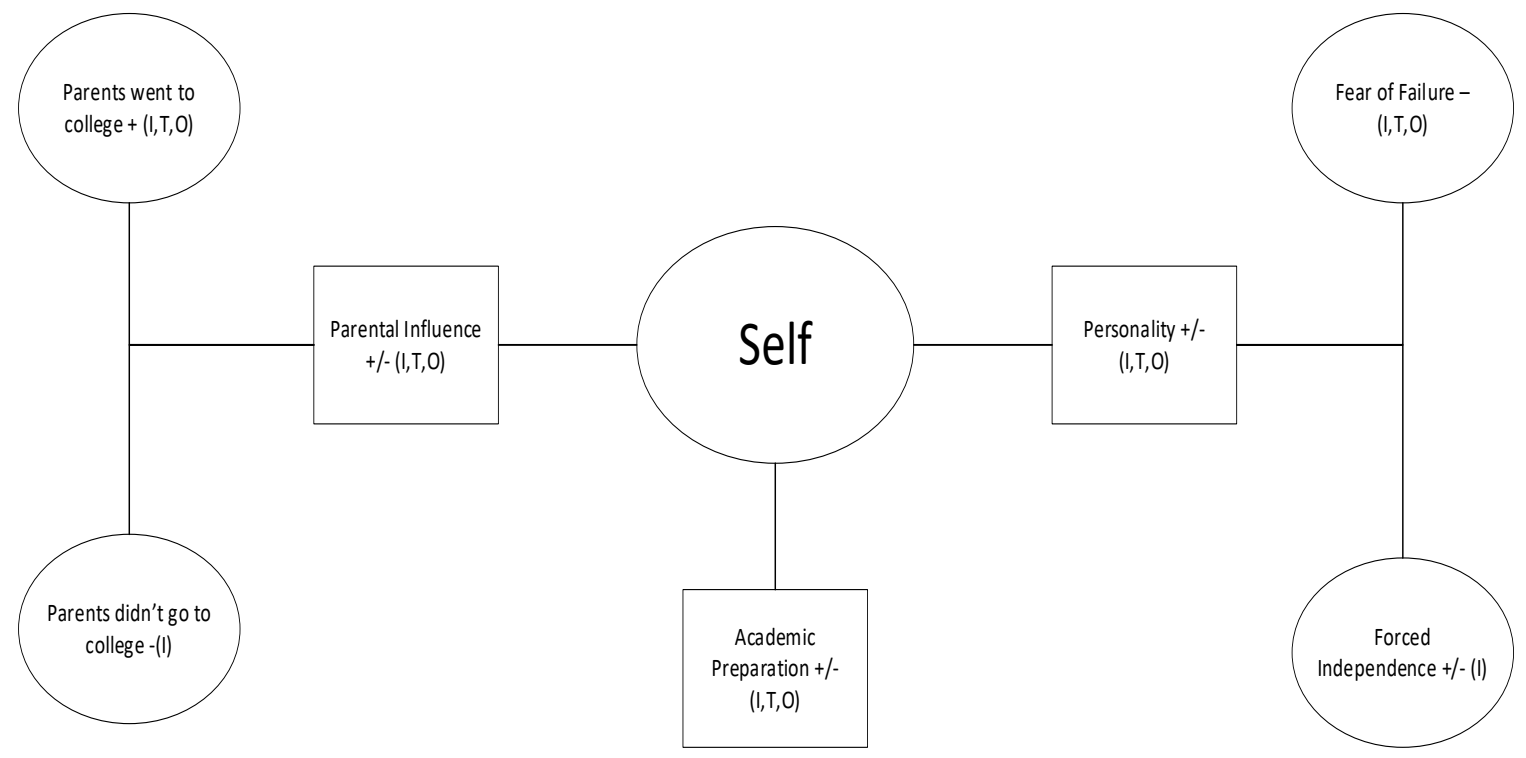

Note. By author 


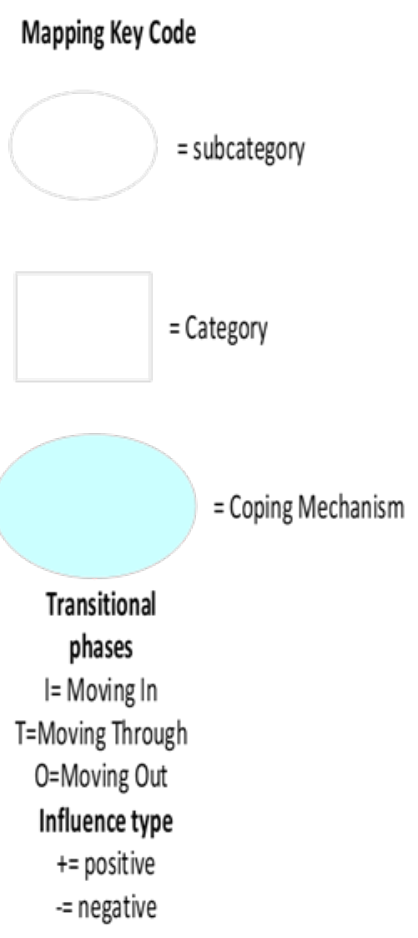

Self

One-on-one and focus group interviews contained three questions specifically designed to address how the "self” influenced the transition to college; these questions can be found in appendices $B$ and $C$ of the interview questions. The self sub category mapping identified three categories: parental influence, academic preparation, and personality. These categories were made up of five sub categories: parents going to college, parents not attending college, academic preparation, fear of failure, and forced independence.

\section{Parental influence}

Sam describes his family’s college going culture “In my household it wasn’t really an option it was understood; graduate high school and you're going to go to college”. Parents who went to college, from a young age instilled the concept and expectations of going to college on the participants. This created a culture of 
support and encouragement. This positive influence was identified throughout the transitional process. Participants described the college going culture as a positive from parents who had attended college. Another sub category of parental influence are parents who didn't go to college. This sub category had a negative influence on the participants. Jessica discusses how her family informed her that attending college would be up to her both financially and academically and how little support was provided during the "moving in" stage of the transition. Parents' educational attainment, either attended or did not attend college, was a major influence on the overall attitude towards college attainment.

\section{Personality}

Fear of failure and forced independence were sub categories which develop the category personality. Many participants described a moment or multiple feelings of being afraid they would fail. Abigail shares "after my first calculus exam and everyone else talking about how easy it was, I remember being so afraid of failing. How would I go home and tell my family I couldn't make it in college?”

\section{Academic Preparation}

A sub category which ultimately became a category is academic preparation. This sub category became a category as it was continuously mentioned throughout the interviews. The category had both positive and negative influences, that could be seen throughout the transitional process. 12 out of the 14 participants described a sense of feeling as if they weren't academically prepared for studying STEM at college. Mary shared how although she has taken AP courses and dual credit courses at her high school, she still felt as if she wasn't 
prepared compared to the other students within the STEM courses. Jack discussed how his academic preparation within chemistry and physics as well as his involvement in project lead the way allowed him to feel comfortable attending courses in college. Jessica shared how she felt academically prepared, so she slacked off but then a few months into college she started recognizing her grades were slipping because she wasn't putting forth the effort.

Categories within the self sub category mapping included personality, academic preparation, and parental influence. These categories summarized groups of sub categories which influence the individual's self. The categories of parental influence, academic preparation, and personality influencing the transition are supported by previous literature. Participants ability to feel as if they should and could attend college, along with the ability to overcome the feeling of failure, forced independence, and their perceived academic preparation all contribute to the individual's self. All categories have the potential to influence the participants' transition in either a positive or negative way. For example, if a participant felt as if their academic preparation was sufficient, they saw their preparedness as a positive impact towards one's self and ability to adapt within the transition. However, if a participant's personality category was viewed in a negative aspect, they found additional difficulty adapting to the transition.

Additionally, the data collected within this study does provide support in the expansion of Schlossberg's transitional theory within the focus of higher education to include academic preparation as this was a reoccurring category throughout the research. By expanding the theory to include academic preparation as a stand-alone 
coping mechanisms, this allows higher education scholars to address the need for appropriate K-12 education. Additionally, Schlossberg's transitional theory should consider expanding to include how academic preparation impacts a person’s cognitive development within the 4S's. 
Figure 6

Thematic map showing support

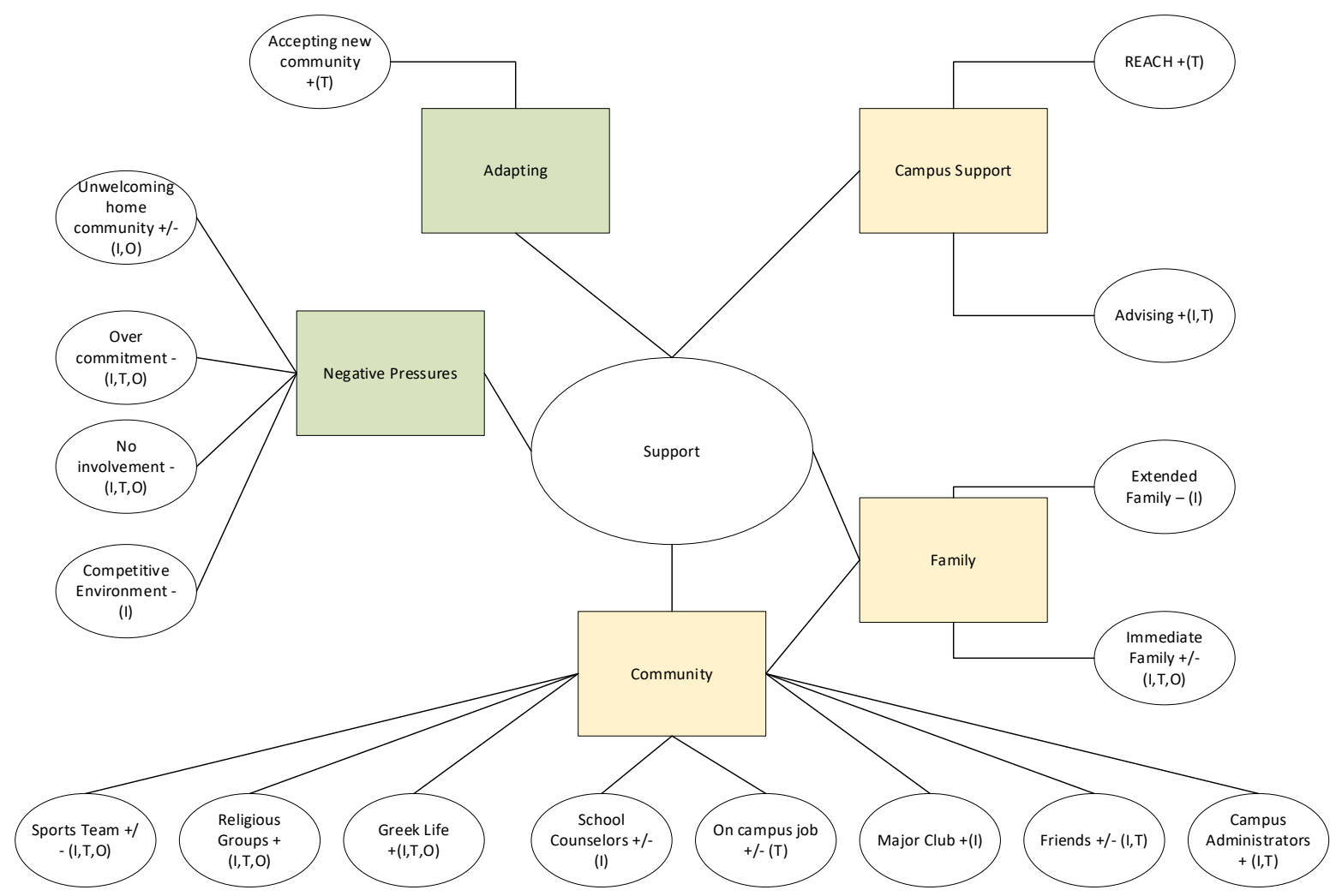

Note. By author 


\section{Support}

Within this study, support was a significant theme reported by AKY students transitioning to college while studying STEM at a urban institution. Support is defined as interactions, feedback, and affirmation between the intimate family, friends, university personnel as well as the individual to assist within the transition (Anderson, Goodman, \& Schlossberg, 2012; Evans, 2010). Five questions were asked within both the one-on-one and focus group interviews to measure the influence of support among the transitional experience of AKY students. AKY students repeatedly stated how they relied on various types of support systems both at home and within their new community throughout their

transition. A total of 17 sub categories were identified which were then combined into five categories. Sub categories within support include: accepting the new community, unwelcoming home community, over commitment, no involvement, competitive environment, sports team, religious groups, school counselors, on campus job, major clubs, friends, campus administrators, immediate family, extended family, advising, and REACH (university tutoring services). Emerging categories included: adapting, negative pressures, campus support, family, and community.

\section{Adapting}

The categories within support were determined by the grouping of sub categories from the first cycle of coding. Accepting the new community was an individual sub category which was moved into the category of adapting. This sub category had a positive impact on the transition while the individual was going 
through the transition and before adapting. As participants were willing to accept the new community and embrace its difference from "home" they saw a positive impact on their transition. As Sam stated as he began to get more involved, he found himself enjoying the new community, although it was different then his home.

\section{Negative pressures}

An emerging category “negative pressures” was identified to capture the experiences of students. Students described the following sub categories: competitive environment, no involvement, and over commitment. These subcategories all provided a level of impact which caused negative pressures within the transition, even if the participant suggested that these negative pressures caused an overall positive influence.

Participants encountered a “competitive environment” was captured within their STEM cohort setting. Participants discussed how they felt as if they needed to compete for the best grades and how STEM courses felt "cut throat.” This was significant in the "moving in" stage of their transitional experience identified as negatively impacting their educational journey. Taylor states "I remember my calculus professor telling us to look to our left and look to our right, half of you won't be here next semester. It felt as if everyone was willing to do anything to make the best grade.”

No involvement is the second sub category which impacts the negative pressures. No involvement specifically discusses the lack of engagement within the new campus community. The no involvement sub category was seen as a negative 
impact and was identified in all three stages, moving in, moving through, and moving out of the transition into college. Participants who didn't get involved their first semester on the college campus saw additional struggles within their transition and ultimate adaptation into college. Participants who did not become involved experienced a feeling of loneliness, isolation, and an imposter syndrome within the university. Denise described how not getting involved increased the difficulty of adapting to college, "College life was such a change, not having any friends made it harder to adjust to campus. I've thought about if I'd gotten involved maybe I could have found study partners earlier.”

On the opposite end of the spectrum, the third subcategory is over commitment. When participants became too overly committed it caused for additional negative pressures within the transitions. Sarah talked about how her self-esteem and belief she could be successful within college was negatively impacted by the over commitments within organizations and the university. The over commitment sub category was found throughout the transitional stages and was identified as only a negative impact. The last sub category within the negative pressures sub category is unwelcoming home community. This sub category was seen during the move in and move out phase of the transition to college, as well as both a positive and negative impact. 12 out of 14 participants stated that they viewed their home towns as unwelcoming and at times as isolating. Yosso (2005) describes family support strongly influences a person's familial capital and their is a correlation between a positive familial capital and success. Kayla describes going home in the following way "I feel out of place because now I have a mix of that 
life and I've been here for four years. So, I have a decent understanding of here and my eyes are opened to a lot more. So, coming back (home) it's just kind of... I'm not comfortable, I feel almost like an outsider but not quite”. Taylor stated that when she informed members in her community, she was attending an urban college and studying STEM they responded with "I love you anyway" and “Louisville is so dangerous...don’t go to those parts, you'll get mugged”. In general participants stated that over commitment played a negative role within the transition as it caused students to become overwhelmed.

\section{Campus support}

The third category is campus support. Campus support was developed from two sub categories: advising and REACH (the university tutoring center). These two sub categories were specifically mentioned as assisting students during the college transition. Although the university provides many resources and campus support services, these two sub categories were specifically mentioned by multiple participants. Advising was seen as a positive influence and identified as being present during the moving in and moving out phase of the transition. Advising was mentioned by 10 out of the 14 participants and tutoring was mentioned by 7 of the 14 participants. Sam stated that his academic advisor was extremely encouraging as he transitioned to college. His academic advisor taught his introduction into college course and met with him on a regular basis to discuss how he was adapting while also providing resources. Tutoring was a positive impact during the moving through phase of the transition. Kayla stated she became a tutor and wished she had attended the tutor sessions while she was a freshman. Additionally, James 
states that he felt as if he could handle the college academic expectations once he started attending tutoring his first semester. The category of campus support identifies that students from the AKY subpopulations have a smoother ability to adapt to the transition if campus support resources are utilized.

\section{Family}

Family is the fourth category within support. This category is comprised of two sub categories: immediate family and extended family. Extended family was identified as a negative influence by participants and appeared only during the moving in phase of the transition. Extended families were defined by the participants as those outside the mother, father, and siblings. Four participants stated that their extended families were not supportive of college in general nor of them attending an urban institution. Additionally, all four participants stated that their extended family didn’t know what careers were available within the STEM field. John stated that he was made to feel, by his extended family, that going to college, and trying to get a high paying job was showing that he was too good for the lifestyle and family he'd grown up in. John described this interaction as causing stress and self-doubt as his family wasn't supportive of his decision. Previous literature suggests that rural communities tend not to be supportive of individuals who strive to do better for themselves or leave the community (Family Relations, 1996). A few of these participants felt this same sentiment. Overall, extended family were discussed to have a negative impact on the transitional experience for the AKY students as they transitioned to college.

Immediate family, however had both a positive and negative influence 
throughout the transitional experience for AKY students coming to college while studying STEM at an urban institution. The immediate family shaping their opinion of college was mentioned by every participant; specifically, each participant mentioned either the positive impact of their immediate family, parents, and sibling(s) or the negative impact by their lack of support. Within the immediate family, the mother was mentioned as being a strong positive influence in the decision to attend and the ability to succeed in transitioning to college. This supports the argument by Dyk \& Wilson (1999) stating that mothers within the Appalachia region play the most influential role on their children's career attainment. Taylor stated that her mother was the greatest motivator in attending college as well as filling out scholarship applications. Sarah discussed how her mom went with her for college visits, helped her fill out applications, and scholarships. Sarah relied on the support of her mother not only during the application process but mentioned calling and facetiming her mother multiple times a day during the first semester. Since the AKY community has strong ties to their immediate and extended family, support is vital in transitional success to college.

\section{Community}

The last category within support is community. This category is made up of the various sub categories regarding places in which participants found a niche or smaller community to identify with. These sub categories, were found throughout the transitional process, had both positive and negative impacts on the person during the transition. The sub categories within community include: sports teams, religious groups, Greek life, school counselors, on campus job, major club, friends, 
and campus administrators. These sub categories in general focused on smaller networks of individuals, outside of their family, which provided positive feedback. Although these sub categories could be positive or negative, in general they provided a positive impact for the ability to adapt to the transition into college. School counselors, friends, and campus administrators are unofficial social groups which participants found support. Jack states that finding a small group of friends from his residence hall when he entered college helped within his transition. He states that these friends became his study group and support system. Again, these sub categories can have a positive or negative impact on the transitional experience of the individual.

Negative pressures weigh heavily on the participants within this study. Specifically, the pressures from over involvement within the new community, no involvement within the college campus, and the extreme competitive nature of studying STEM. In addition to adjusting within the new community, participants mentioned their old community, AKY, became unwelcoming and, at times, would put down the participant for their success in transitioning to college. Mary stated "One of my friends, he's super country. And he was like...oh you think that you're so much better than us now because you're one of those big city folk, and you came back. And now what do you think you're...you're smarter than us.” 
Figure 7

Thematic map showing situation

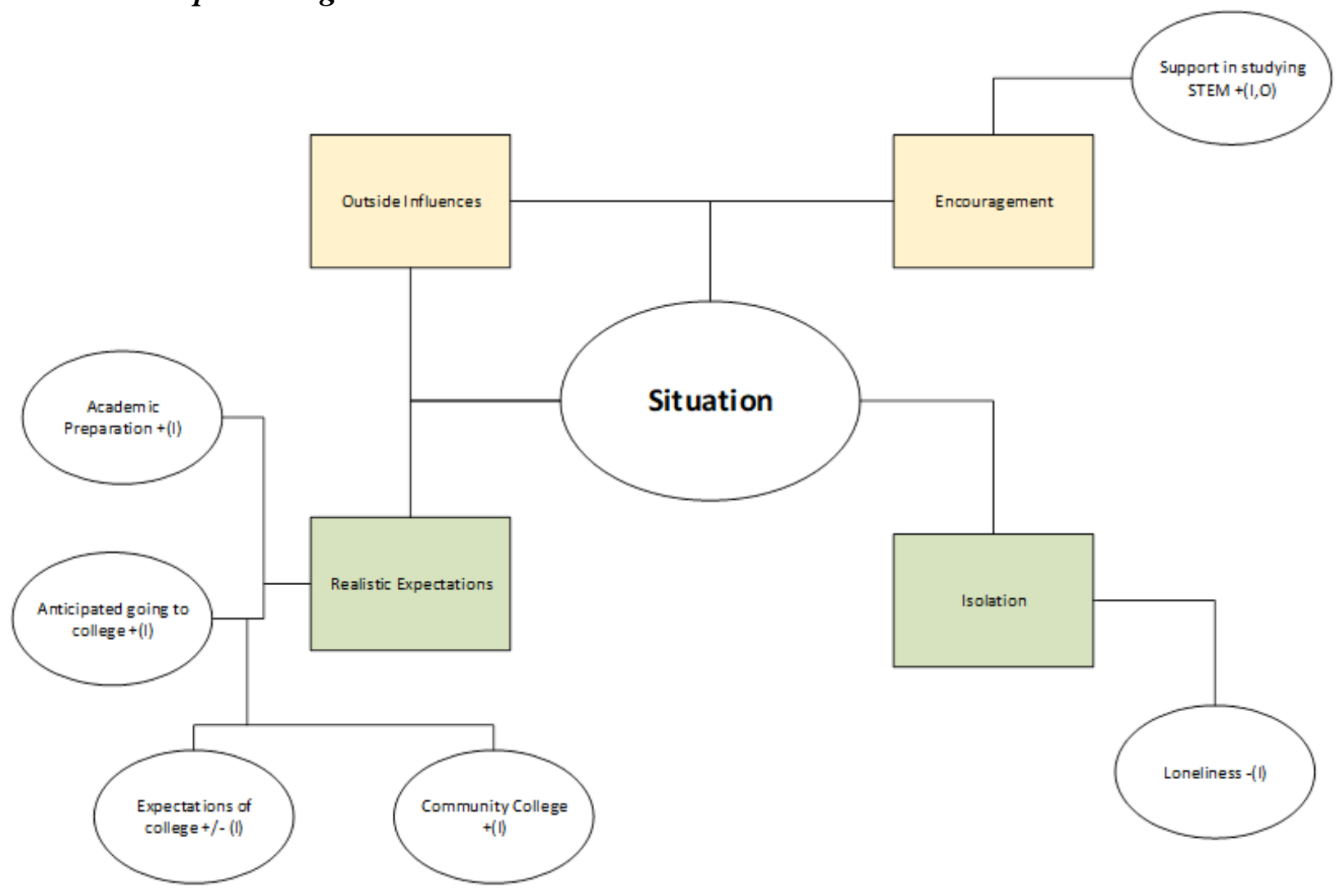

Note. By author 


\section{Situation}

A third emerging theme "situation" captured four different categories: realistic expectations, isolation, outside influences, and encouragement.

\section{Realistic Expectations}

Realistic expectations ensure that the student understands what transition will happen, how to adapt, and areas in which they need to adjust to have a successful transition. Of all the interviews, $100 \%$ of participants described situation, sub categories as academic preparation, anticipated going to college, expectations of college, and community college experience to describe their transitional experience.

The first sub category within realistic expectations is academic preparation. This is an aspect of feeling either academically prepared or behind their peers and an incessant comparison that affects their transition. Every participant discussed making these comparisons of being academically prepared or under prepared with their peers. There was evidence linking students who felt they were academically prepared to an easier transitional experience. James shared the following "I felt I was at an extreme disadvantage to my peers and I know they say you can drop back and take the pre calc classes and everything but to be quite honest I was too proud and too competitive to do that. And they (pre calc course) would have helped me probably in the long run, yes. I would've had a better foundation of everything. But I also had a fear that...if I did that it would set me back a semester... (and) I might become isolated from my peers”. Academic preparation provided both positive and negative aspects within the "moving in” transitional period. While 
$100 \%$ of participants discussed their perceived academic preparation, 12 of the 14 participants mentioned the feeling of under preparedness academically.

The participants who described anticipating college, saw it as a positive experience and assisted in their transition to college. The anticipation of college was created through the immediate family instilling in the participants that college was a positive option and an experience in which one should be excited. Taylor states "I would say my mom was my biggest motivator. She helped me with all applications. She took me to Western, UofL, UK and Northern to visit. She got me on the right track... like really early because a lot of people wait but she got me to apply really early". The sub category of anticipation of going to college was described as a positive sub category throughout the moving in process of the transition.

The third sub category within the realistic expectations category is college expectations. Participants described this as both a positive and negative factor within the moving in process of the transition. Understanding what to expect as a participant leaves home and enters a new culture allowed for students to perceive their adaptation to the transition. Participants described feeling more confident and at ease with the transition when they had realistic expectations of what the culture will entail. On the other side, participants who didn't have realistic expectations described struggling throughout the moving in process of the transition.

Students shared the community college sub category emphasized the importance of setting realistic expectations. This sub category was a positive factor during the moving in phase of the transition. This factor was mentioned by one 
participant, but this individual expressed the major impact that community college had on adapting to the four-year college. Denise stated that the community experience prepared her for a four-year college experience more so then any other academic experience. Denise went on to state that the less stressful environment of a community college helped with the transition to the "big college experience".

\section{Isolation}

The second category is isolation. Isolation appears to be a negative aspect of the transition and creates a more difficult route to adapting within the transition. This category has only one sub category, loneliness. Loneliness was described as a negative factor during the moving in phase of the transition. Loneliness was described by participants as not feeling as if they belonged in their home community or their new college community and feeling as if they are alone in the transitional process. Kayla states "Some people never leave the Ashland Greenup area see those that graduate high school and go to the big city for college and come back as if they are better than others now because you have education". She went on to share that when people at the college find out you are from eastern Kentucky, they look at you differently, they negatively

judge you. The participants who experienced isolation shared how feeling alone and isolated created a more difficult time in transitioning to college.

\section{Encouragement}

The third category contributing to the situation theme is encouragement. Participants who experienced encouragement throughout the process of the transition had a positive impact on their adaptation. Participants identified support in studying STEM as a positive sub category which impacted the moving in and moving through process of the 
transition. Participants who identified support in studying STEM found their transition to be smoother. Participants described encouragement from their parents, school counselors, and siblings as a positive experience in both selecting to attend college and studying STEM. Abigail described receiving a D on the first Calculus exam and how she reached out to her parents. Abigail recalled the conversation with her parents, “you have to work hard but you also have to do this for yourself”. They provided encouragement throughout her entire transitional process into college.

\section{Outside Influences}

The last category is outside influences which is classified as an external factor. Outside influences were mentioned by the participants as both positive and negative factors which are found during the entire transitional process. Participants mentioned outside influencers as their community (both home and college campus) and life situations. Participants described that when community support occurred the transition was smoother throughout the entire process. Additionally, participants described life situations as having a negative influence on their transition into college; these situations included financial hurdles, sickness within the immediate family, and adapting to a larger city environment. Georgia described how being a woman in STEM brings its own barriers "being a woman in STEM you need to understand that there are gonna be people who say you can't do this because you're a female or from the country”.

Within this overarching theme (situation), internal, and external influencers began to arise. Situation contains four categories: realistic expectations, isolation, encouragement, and outside influences. These four categories contain a total of seven sub categories which were identified by the participants. Although situation 
played a role on the transition of participants, it was not the greatest impact on the ability to adapt to the transition. 
Figure 8

Thematic map showing strategies

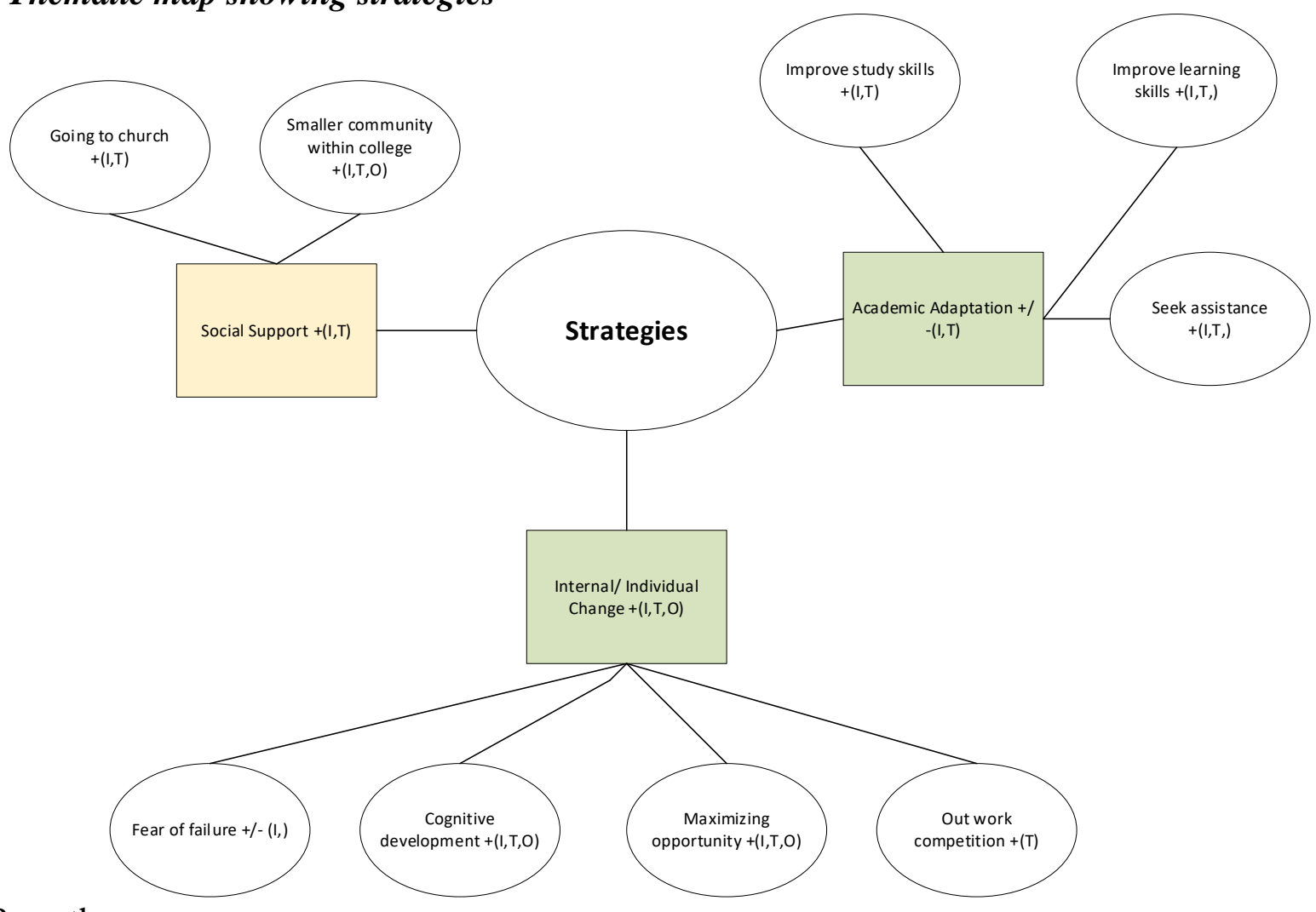

Note. By author 


\section{Strategies}

Strategies are the actions which assist an individual within their transition. A total of nine sub categories were collected within strategies. These sub categories were then collapsed into three categories: internal/individual change, academic preparation, and social support. Additionally, academic adaptation and internal/individual change were internal factors while the category social support had external factors.

\section{Internal/Individual Change}

The first category within strategies is internal/ individual change which includes four sub categories: fear of failure, cognitive development, maximizing opportunity, and out work competition. These sub categories focus on the individual adjusting their perception or opinions and developing determination to succeed within the transition.

Fear of failure is the first sub category within internal/ individual change. Participants described a feeling of being overwhelmed and failing, this perceived opinion of failing was centered around failing at school. Specifically, they were worried about not being successful in college, having to return home and being a failure. Participants discussed fears of failing a class or being an imposter within their major. Fear of failure was expressed to be both a positive and negative factor as participants transitioned into college. Students planned to either use it as an opportunity to refocus and persist or to focus on that fear and potentially struggle within the transition into college.

The second sub category within the category of internal/individual change was cognitive development. Cognitive development is the development of skills, 
knowledge and problem-solving abilities within an individual. Participants expressed the ability to develop their study skills, time management skills, and selfawareness as positive factors in transitioning to college. However, students who were not able to focus on cognitive development found additional struggles and barriers. Abigail describes self-esteem and skills development in the following manner "I was destroyed and totally embarrassed and that's half the reason I wouldn't go to class anymore because I would just walk out of a test and these other people that I knew a little about were just oh I didn’t think that was so hard and I would just cry and run home and not come out of my room. It was unhealthy. Going to counseling and taking a lot of time (helped). It was a lot of just changing the mindset because I kind of just thought why (try) if I'm going to fail anyway”. Abigail went on to state "I just really didn’t want to give up and it really made a difference to know...there isn’t a backup plan. So, stop worrying about it and just keep trying and that's kind of something my parents just helped out a lot.” The ability to engage in cognitive development has the potential to affect the transitional period negatively and potentially even delay the progress from one transitional phase to another.

The third sub category within the category of internal/individual change is maximizing opportunity. This sub category is seen as a positive for participants who were able to take advantage of the opportunities they received either from their home community or the university setting. These resources included tutoring services, support from university administration, academic advisors, activities around campus, and community resources. Participants who attended workshops on success, saw an increase in the ability to adapt to the transition. This sub 
category was seen throughout the moving in, moving through, and moving out process of the transition to college. Taylor describes frequent visits to her academic advisor not only to discuss course work but other aspects of attending college. Taylor shares how she went to her academic counselor to discuss financial issues and roommate conflicts during her transition into college. Her academic counselor also encouraged her to visit other activities to meet friends as well as developmental programs to assist in her success at college. Overall, there was a direct correlation between participants who maximized opportunities and those who had a smoother time adapting to the transition into college.

The last sub category within the interval/individual change is the concept of out working the competition. A majority of participants expressed either an actual or perceived lack of academic preparedness, lack of self-esteem or overall concern of failure. However, of those participants and those who did not express these feelings they all shared a concept of using their "Eastern Kentucky" roots to out work the competition. Interviewees shared a sense of pride from the students in the ability to work harder and longer to find success. Mary describes how the social aspect of college was affected by out working the competition "So a lot of social stuff and the internal type stuff that was happening to me was put on the backburner because I was so worried about school. All I did was study, go to tutoring and study groups. So, I didn’t really deal with it (the other aspects outside of course work) I just kind of let it happen the way it was happening. I was like oh it's not that big of a deal because my main focus is school. It's all these other problems I might be having (such as) I'm feeling weird being in this new community or I'm feeling all these emotions...I cared about my grades, throwing myself into school 
so that I could out work everyone else”. Mary goes on to discuss how experiences such as her sorority, study abroad shaped her into the person she is today.

\section{Academic Adaptation}

The second category within the strategies, a factor within Schlossberg's 4S system, is academic adaptation. Academic adaptation is the ability to adjust to the academic expectations of a STEM major. This category was viewed as having positive and negative factors during the moving in and moving through process of the transition. This category had two sub categories: seek assistance and improve study skills.

The first sub category within the academic adaptation category is seeking assistance. This as described by the participants as the ability to recognize when help is needed to seek out that assistance. Every participant at some point during the moving in and moving through phase of the transition mentioned seeking out assistance when they hit a hurdle. This seeking out assistance may have been tutoring, an academic counselor to navigate adjusting their schedule, or financial aid regarding scholarships, etc. Denise states the following regarding finding assistance from her academic counselor "I had GEN 101 with my advisor. That was a good first half semester class to start out with because I got to know her really well and I mean we went through some pretty cheesy (stuff), but it helped. I went to her long after that class for help on everything.” Participants who mentioned seeking assistance saw it as a positive factor during the moving in and moving through phases of their transition into college.

The second sub category within the academic adaptation category is "improve studying skills”. This is described by participants as taking specific steps to adjust and enhance their studying skills. Participants mentioned having to relearn how to study and that they needed to take advantage of studying sessions provided by the university. This 
subcategory has a positive factor during the moving in and moving through phase of the transition.

\section{Social Support}

The third category within strategies for transition success is social support. Social support are external factors which provide encouragement and assistance to the participants. Social support if utilized by the participants were always described as a positive factor throughout the entire transition into college. Two sub categories were identified within the category of social support: going to church and finding a smaller community within college. The social support category within strategies is an approach to develop a support system to assist within the transition to college. As stated in the previous diagram, support has been identified by the participants as being significant within adapting to the transition into college. Creating a new social support system, a smaller community within the larger university was identified as a positive factor to assist within the transition.

The first subcategory within social support is creating a smaller community within the college. This sub category was a positive factor and participants described its importance through the transitional phases of the transition. Jack describes finding a smaller community strategy in the following way "coming from that small community to such a large campus and a large city it was difficult but finding that small knit community within campus, I feel it really helped.” Abigail shares "home versus here just has an entirely different atmosphere and feeling I could find my niche here and sort of fabricate what it felt to be home”. In general, the participants who shared they were able to develop a smaller community within the college atmosphere, had a smoother transition. 
The second subcategory within social support is joining a university church. Although this could have been coded as joining an organization collectively with involvement or other student organizations, “going to church” was kept separate due to the importance religion plays within the participants home community and the focus on religion not being viewed as a student organization but as a core value to the individual. As participants described joining a university church, they connected the membership to feeling as if they were emulating a home community. The participants described the connection to their church differently than joining other organizations; specifically joining other organizations were adapting to the new culture and norms within college. Whereas joining church was described as an approach to stay connected to their home community. Sarah describes it as "joining the church on campus allowed me to feel I was back at home, with people who came from my community”. Participants who joined a church on campus viewed it as a positive factor during the moving in and moving through phases of the transition.

\section{Transitional phases}

The 4S system within Schlossberg's transitional theory all were described to play a role within the transition. However, in reviewing the various S's (self, support, situation, and strategy) support is described as the most influential in terms of adapting to the transition to college. Participants who shared having a positive experience with their support system, they also described having a smoother transition into college. Although they still experience negative factors within the influence, they did note that overcoming those influences and feelings didn’t have detrimental effects on their success within the transition. 
Analyzing Schlossberg's 4S coping methods codes were identified during the first coding cycle. During the transitional cycle sub categories were merged together into categories within each coping method.

The first $\mathrm{S}$ is self. Self is defined as "personal characteristics such as socioeconomic status, gender, age, ethnicity, health, self-esteem, outlook, commitment, values, spirituality, and resilience within self” (Evans, 2010, 217). This research identified three categories within self: parental influence, academic preparation, and personality. The three categories were developed through the following sub categories: fear of failure, forced independence, academic preparation, parents didn't go to college, and parents went to college.

The second S is support. Support refers to the level of positive interactions, feedback, and affirmation between the intimate family, friends, university personnel as well as the individual to assist in transition (Anderson, Goodman \& Schlossberg, 2012; Evans, 2010). This research identified support as being the biggest influence within the transition to college. Additionally, five categories were developed during the transitional cycle of the coding: adapting, negative pressures, community, family, and campus support. These categories were developed from the following 17 sub categories: accepting new community, unwelcoming home community, over commitment, no involvement, competitive environment, sports team, religious groups, Greek life, school counselors, on campus job, major club, friends, campus administrators, immediate family, extended family, advising, and REACH (tutoring center).

The third S is situation. Situation describes how a person evaluates the transition and determines their level of control over what is happening (Evans, 2010). Situation identified four categories: outside influences, encouragement, realistic expectations, and 
isolation. These categories were developed during the transitional cycling based off first cycle coding. The first cycle coding included the following: support in studying STEM, loneliness, community college, expectations of college, anticipated going to college, and academic preparation.

The fourth $\mathrm{S}$ is strategies. Strategies are actions that lead to the ability to manage a transition (Evans, 2010). Strategies identified three categories during the transitional cycling: social support, academic adaptation, and internal/individual change. These categories were developed based off the first cycle coding and included eight sub categories: going to church, smaller community within college, improve learning skills, seek assistance, out work competition, maximizing opportunity, cognitive development, and fear of failure. Upon completion of this coding cycle, a second coding cycle was completed utilizing a composite sequence analysis.

Since this study utilizes a pre-established framework, elaborative coding is the best approach in the second cycle of coding. The categories were established within a composite sequence analysis and revealed four major themes: 1 . Social support with deeper relationships, 2. Lack of academic preparation for an intense R1, urban STEM school, 3. University support within social support in assisting an individual, and 4. Individual adaptation. 


\section{Figure 9}

\section{Transitional phases diagram}

Composite sequence analysis: Transition through first year of college Participants: 14

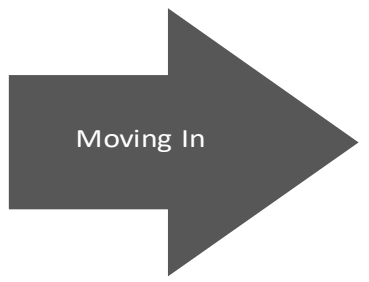

Easy transition $(\mathrm{n}=8)$

Support, family,

community, adapting,

realistic expectations,

encouragement internal

change, academic

preparation
Difficult transition ( $n=6$ ) Isolation, lack of: realistic expectation, academic preparation, academic adaption, social support, internal change

Themes:

Support, Self, Situation, \& Academic Preparedness

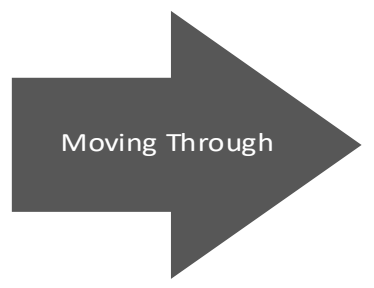

Day to day management

\section{$(n=9)$}

Parents college education, personality, family independence, community, adapting, encouragement, internal change, social support, academic adaption
Not able to manage day to day $(n=5)$

Negative pressures, family, community, unrealistic expectations, outside influences, internal change

Themes:

Self, Support, Situation \& Academic Preparedness

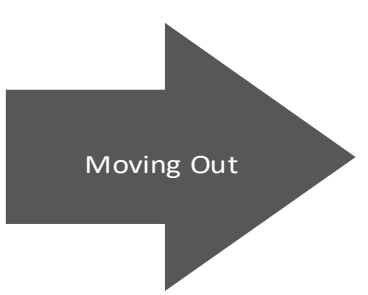

Adapting to college $(n=12)$

Parents college education, internal change, academic preparation, adaptability, community, family,

encouragement, social support, development of success skills
Not adapting to college

$(n=2)$

Family educational support, internal change, negative pressures, community, family

Themes:

Self, support, situation, strategies \& Academic

Preparedness

Note. By author 


\section{Social Support with deeper relationships theme}

All participants discussed the importance of finding friends and creating a strong support group. These relationships need to be deep and share core values to assist within the transition. Those participants who expressed being able to develop a strong social support system, either familial, or with peers expressed an easier transitional experience. Taylor discussed the difficulty of transitioning when you lack a familial support system at the institution: "But I hated Louisville my first year (but) I totally consider it my home now. I've been here three years. It's great. I hated it because...it was such a big transition.” Taylor went on to say, "freshman year. I had a lot of calls home to my parents whenever I was having a really rough time or something and that did help to a point. But it's also at the end of the day they're not here they can't help you get through whatever you're trying to get through. So, in the end it was just friends I made in the dorm and on teams that helped a lot.” James struggled in finding a support system and became over involved on campus. James discussed "I just joined every organization I could, I think I was involved in seven my first semester. I focused too much on being in organizations that my studies and grades suffered.” A strong support system, either through immediate family or the development of support at the university was an influential factor within the transition and eventually the adaptation into college. Participants using their support system occurred throughout the moving in, moving through and moving out phases of the transition. The strong support system provided encouragement, guidance, assistance, and resources which aided in the adaptation to college. Having a strong support system with deep commitment to the students' success will be important in the success of rural students transitioning into a new environment and within STEM education. 


\section{Lack of academic preparation}

Academic preparation within math and science is a key factor in success within STEM higher education. Participants discussed the struggles of transitioning academically; going from being a top student at their high school to feeling academically inadequate within college. Mary discusses this struggle: “Academically I don't think I was prepared at all to come to college and I don't think it's really the fault of the faculty because you only have so many resources. Obviously high schools like Manuel are going to have a ton more resources (than) Rock Castle County. But yeah, I didn't feel, I didn't feel prepared.” This student describes how the lack of exposure to higher level educational theory and deep learning created a barrier within her transition into college. Participants discussed how the perceived lack of academic preparation negatively affected the ability to adapt within the transition. This is significant within the realm of self, support, and situation during the transition. John also shares how the rigor of high school didn't prepare him for college: “I didn't feel challenged as much as I should have been probably which is why I had a really hard time transitioning into college because I didn't, I just didn't have the background information like know how to study here”. Jack had this to say “I didn’t have to study for anything to get A's. If you're at a certain level and that's the problem with I think a lot of AP kids in high school is they get in trouble when they come to college because you get real cocky in high school and they're bad at studying and then they carry that into college. They might be able to get away with that for a semester like I did. But in that second semester, classes actually started (getting tough) there's no more review. You're learning and at a fast pace.” 
Participants who perceived as if they were academically prepared for the rigors of a STEM major, presented their transition as smoother with a quicker time to adapt. These participants didn’t express imposter syndrome or a feeling as if they didn’t belong among their peers. However, the participants who perceived their academic preparation as inferior to their peers express a tougher process transitioning to college. These participants described the added pressures of "not feeling smart enough” to the already stressful transition into college. Additionally, there was a positive correlation between participants who felt as if they weren't academically prepared and lacked strong study skills. Academic preparation has been studied and identified as a significant factor to college success; however, this study has also identified it as a strong influencer on the success of transitioning to college as well.

\section{University support within social support/ assisting an individual theme}

Universities provide a variety of levels to support incoming and current students. Participants discussed how they utilized both student services and student activities on campus. Jack expressed how the campus within the city acts like a small society, similar to his home town. Kayla describes how she was able to find support academically within the university, which assisted in her ability to be successful within her transition. Although participants should be careful to not become overly involved; engaging within the campus community provides the ability for participants to enhance their coping mechanism skill and support systems.

Universities and professionals need to ensure they are providing individualized programming to assist with the transition to an urban institution from 
AKY. Providing multiple avenues for students to successfully transition can be achieved through universities assisting the students in discovering who they are, how to cope with transition, recognize their situation, and develop strong support systems. These programs, policies, and interventions need to be focused on building a support system among peers, family support as well as academic preparation. This could look like informational sessions for the parents to discuss how to be supportive during a transition or focusing this subpopulation on a math academic prep course. If universities plan on placing a targeted effort on recruiting rural students, they should be obligated to provide programs and policies which encourage a sense of success.

\section{Individual adaptation}

Individual adaptation encourages the student to change and grow in a way that will allow them to succeed at college. The participants mentioned avenues of out working their peers to succeed in college and how the fear of failure must be pushed out of their mind. As the individual accepts their transition and adapts within their new community, they develop strategies, and attitudes to succeed within their transition.

\section{Limitations}

Although the analysis of chapter 4 provides great insight and valuable knowledge regarding the transitional experience of a subpopulation, it's not without limitations. Due to this study having a case study approach it is not generalizable (Creswell, 2009). This study is also limited to one urban institution. Additionally, Schlossberg's transitional theory is widely accepted within higher education, however it provides only snap shots of an individual's transition and lacks a concrete understanding of the adaptation to the 
transition. Schlossberg's theory is complex and subjective, this leaves a lack of a formal assessment tool for the theory. Schlossberg's theory also lacks utilization within this subpopulation in higher education research (Evans, at el, 2010). Schlossberg's theory directs the reader to assume that transitioning follows the flows of moving in, moving through, and moving out; however there lacks the process of if an individual reverts back to a specific phase within the transition. Transitioning is highly individualized process, however this theory lacks any suggestion of timeframes which should be spent within the transitional phase. Additionally, due to the continuous expansion of the theory there lacks an instrument for measurement of reliability. Although Schlossberg's theory is accepted across various disciplines because of its generalizable conceptual approach, however this leaves specific factors which affect a transition into college out of the equation. A specific example of this is academic preparation. Research indicates that academic preparation plays a role within a students ability to retain at a university, yet it's left out of Schlossberg's transitional theory.

\section{Conclusion}

This chapter examined data to determine emerging themes and correlations. The analysis of this data occurred through cycle 1 coding, transitional coding, and cycle 2 coding. The data moved from sub categories, to categories, and into themes utilizing the $4 \mathrm{~S}$ system (self, support, situation, and strategy) as the framework. First cycle coding utilizes the $4 \mathrm{~S}$ system as a framework for coding, the second cycle coding utilizes those themes through the lens of the transitional phases (moving in, moving through and moving out). Four themes emerged from the data: strong support system, academic preparation, university focused support, and individual adaptation. The next section will focus on future research opportunities, 
practical implications as well as conclusion to this study. 


\section{CHAPTHER 5: CONCLUSIONS/ IMPLICATIONS}

The purpose of this study was to examine how rural students experience their situation, self, support, and strategies while moving in, through, and out of their transition to college while studying a STEM major at an urban institution. This study adds to the current literature by contributing qualitative transitional research for STEM education among rural students into an urban university, as well as expanding the literature within transiting to college and rural college students. There are multiple implications for various stakeholders including students, families, schools/communities, and the universities the AKY students will be attending. These semi-structured interviews and focus groups provide a cross case analysis highlighting four major themes: Social support system with deeper relationships, lack of academic preparation for an intense R1, University support within social support, and individual adaptation. This study provides insight into the expansion of Schlossberg's transitional theory, as well as focus areas to increase success among students transitioning. Chapter V provides future research opportunities, recommendation of policy and practice, and conclusion.

\section{Future Research Opportunities}

\section{Expansion of Schlossberg's transitional theory into college}

Schlossberg's transitional theory has been an accepted theory among higher education scholars. Although this theory has been expanded throughout the years, additional review and expansion should occur. This theory alludes that each S within the 
4S system is weighed equally throughout the transitional process. However, throughout this research participants have expressed support having a stronger impact than any other mechanism. This researcher believes there's a strong correlation among growing up with a strong community support system and the need for a strong support system during their transition to college. The participants stated they utilized their support system during difficult times and situations where barriers existed. Participants shared that their support system provided positive reinforcement as they developed study groups, and their support system provided knowledge and resources regarding a successful transition. The present study also found that academic preparation was a significant theme as a result of participants sharing the negative impact perceived by a lack of academic preparation they had on their transition to college. Additionally, this transitional theory has the opportunity to be expanded into a fluid theory instead of snapshots throughout the transition.

Schlossberg's transitional theory, with regards to higher education, needs to consider the adoption of academic preparation into the theory. The academic preparation factor within a student's transition, and ultimately their college success has been extensively studied. In terms of the utilization of this theory within higher education, this factor should standalone within the coping mechanisms of the transition. To not highlight academic preparation as a coping mechanism, as the theory does with support or situation, sends a message reducing the importance of its influence regarding a successful transition. 
Table 4

Proposed new 4S system

\begin{tabular}{|l|l|}
\hline Self & $\begin{array}{l}\text { personal characteristics such as socioeconomic status, gender, age, } \\
\text { ethnicity, health, self-esteem, outlook, commitment, values, } \\
\text { spirituality and resilience within self }\end{array}$ \\
\hline Support & $\begin{array}{l}\text { the level of positive interactions, feedback, and affirmation between } \\
\text { the intimate family, friends, university personnel as well as the } \\
\text { individual to assist in transition }\end{array}$ \\
\hline Situation & $\begin{array}{l}\text { an individual's evaluation of their transition and determines their level } \\
\text { of control over what is happening }\end{array}$ \\
\hline Strategies & $\begin{array}{l}\text { actions that lead to the ability to manage a transition. Three phases of } \\
\text { adaptation within a transition have emerged: modify situation, control } \\
\text { meaning, and manage stress in the aftermath }\end{array}$ \\
\hline $\begin{array}{l}\text { Academic } \\
\text { Preparation }\end{array}$ & \begin{tabular}{l} 
advanced academic exposure within the math and science realm \\
\hline
\end{tabular} \\
\hline
\end{tabular}

Note. By author

Additional research should be conducted on the possibility to adapt the transitional theory focusing on subpopulation factors within the transition to college.

\section{Implications for research}

As described above, research on student success is widely available. While this study provides contributions to the existing knowledge of college transition, as well as the subpopulation of rural students, STEM student success, and transitional research. The following recommendations are aimed at bridging the gaps within this body of work:

1. Replication of study. To increase the validity of the findings, it will be vital for researchers to replicate this study. There is an opportunity to conduct this study with a larger sample size through including additional universities and extending the population to other types of STEM settings. It should also be noted that although computer information science students were recruited, there were no participants from that particular major.

2. Real time observation of the transition. This study collected reflection data from a majority of the participants. Participants who were in their sophomore 
to senior year allowed for a strong reflection and mature review of their personal transition; however, the ability to observe the transition as it takes place would assist in furthering the research gap. Additionally, studying the transition in real time allows for the participant to journal and share their feelings as well as coping mechanisms in real time. Real time observation has the potential to enhance the quality of the qualitative research.

3. Urban institution expansion. This study focused on one urban institution. Additional research could focus on universities across the state which offer STEM majors and may or may not be urban institutions. This research has the possibility to provide insight on if rural students find more success in their ability to adapt to college while studying STEM at a non-urban institution.

4. Longitudinal study. Schlossberg's transitional theory states that the process of a transition and the recognition of a transition is solely up to the student. Research conducted through a longitudinal study could provide insight on the long-term impacts of the participants' ability to adapt within the transition. This study would begin collecting interviews during the first semester and allow students to journal their experience throughout the first two semesters. Additionally, the researcher would collect data through follow up interviews once a year until graduation or the student leaves the university.

5. Gender Focus. Additional research and data collection on female AKY students to explore if the impact on support could be gender-specific or community-focus. A gender focus study could also allow for the examination of imposter syndrome, feelings of fraudulence and a lack of confidence in their ability (Chapman 2017), among female AKY students. 


\section{Recommendations for policy and practice}

The findings of this study also inform recommendations for the university, the individual, families, and the hometown community. Although this study focused on the individual experience of transitioning, the recommendations to enhance the ability for AKY students to adapt to college should be focused on the University, as the university has a responsibility to lead these initiatives to increase the likelihood of success for this sub population.

\section{Universities}

Universities have the responsibility to lead the initiatives which ensure that parents and students are prepared for a successful transition into college. Universities have the potential to provide programming which builds community and social support which will increase the successful transition for college students (Mattanah et al., 2010). Universities will need to become invested with the success

of their students and commit to support within AKY communities, faculty and staff trainings, and program evaluations examining for inequality among the AKY community.

Universities should work with high school guidance counselors and community advocates to ensure that students are both aware of STEM careers and higher education institutions that offer STEM degrees. University admission counselors and high school guidance counselors need to work together to provide continuous support of educating the families and communities on the resources available for college students. Hosting informational sessions and marketing campaigns will be vital in educating AKY families on the benefits of a STEM career. 
Faculty and staff will need to invest time and resources in the success of AKY students. This investment should include training for faculty and staff to understand the background of this sub population and potentially removing the stereotypes and assumptions. The university should develop intervention programs and bridge programs to ease the non-academic barriers within the transition into college. These trainings and involvement within intervention programs should be included in the faculty work load through review of faculty annual work plan and tenure trace process. In addition to training, universities should evaluate which dual credit programs within the AKY region to ensure that advanced science and math courses are being offered.

In addition, evaluation of programs to ensure equity for AKY communities should occur both within institutional-programming and policies. For example, institutions could asses the need for financial assistance to families enabling their attendance during residence hall move-in days or family weekends, and if funding is allocated to facilitate the attendance at such events, periodic review of the success of such resource allocations should be conducted. Additionally, the time this program is offered should be reviewed to ensure AKY have the availability to attend. For example, many AKY communities host their county-wide festivals in the fall, do those conflict with family weekend? Could universities look at offering family weekend at times that are better suited for AKY families to attend? Review of financial support for students to attend campus visits should also be evaluated. Universities should look into virtual campus tours as well as programs which sponsor a bus from school districts to bring high school seniors on to campus. Universities need to enhance parent engagement and provide educational programs 
to the family; this can be achieved by hiring community coordinators to provide programs and resources. AKY families should have a point of contact within the university that they can reach out to and discuss how to best support their student. Additionally, universities need to provide literature on the skills needed for students to be successful in their transition and resources on where parents can recommend their students go for help. Universities cannot assume that parents know how to provide support for their students or that parents are aware of the resources which help a student transition into college.

Before universities begin to focus on retention of AKY students, they should evaluate their efforts of recruiting. This population needs to have a specific focus on community relationship building, focusing on STEM college opportunities from a young age. Universities need to develop strategic plans to integrate within the community and create brand recognition.

\section{Students}

As AKY students prepare for their transition, they must understand the various challenges that may exist. Realistic expectations are key to a successful transition as this allows one to prepare appropriately for a successful transition (Gall, Evans, \& Bellerose, 2000). It is not enough that students make the decision to attend college, they must also master becoming a college student (Collier, \& Morgan, 2008). Students must understand the expectations of being a college student and what skills are needed for success. AKY students should utilize the skill development and transitioning programs hosted by the university.

Students should prepare academically as much as possible in their math and science education by taking advantage of duel credit courses, advance placement, 
and extracurricular STEM programs. For instance, Morehead State University offers a program called Craft Academy that serves high school juniors and seniors from eastern Kentucky to provide in depth STEM education and preparations for college. Students should also take advantage of extracurricular activities outside of the classroom. These outside of the classroom experiences include summer internship programs, campus visits, extracurricular activities with a focus on STEM, and volunteer opportunities within the STEM careers. Not only will these advanced courses and STEM experiences prepare the students academically (Hudacs, 2017), it has the potential to increase their perceived esteem of being academically prepared.

Students need to ensure they have the ability to build strong support systems with deeper meaning than just friends. Students should strive to have a good understanding of the support and resources available through the University. Utilizing home community values to assist with the transition and adaptation to college by encouraging students to find smaller communities within the university.

\section{Families}

Positive or negative support within the student's family impacts the transitional experiences (Lararowicz, 2015), which was reinforced through this study. Families should provide knowledge regarding college which can help students leverage their success (Yosso, 2005). Families must understand the complexity of transitioning to an urban institution and a STEM major. There is a need for an extra level of support to ensure students can move in and out of the transition successfully. The results from the present study found that families should focus on the following aspects to support their student: positive 
encouragement, exposure to city/ campus, connection to High School guidance counselor and teachers, and university engagement,

Families have the potential to support their AKY child from a young age through college. A college-going culture during the students childhood needs to occur for students to have college ingrained within themselves; families have this opportunity to encourage their children to begin thinking about college as an option after high school. This level of support has to continue beyond formative years and throughout their college experience. As students face barriers within the transition, it will be important for families to utilize the positive support and resources they have available.

Families need to consider providing exposure to the city and campus visits as their children consider attending an urban institution. Research shows the potential for increased emotional stress due to lack of exposure to large cities (Hudacs, 2017). This exposure before transitioning to college, can lead the student to feeling more confident about the situation as well as gain realistic expectations of city life and an urban college.

Building partnerships and alternate forms of social capital such as familial capital (Yosso, 2005) will be vital for families to support their students. The relationships families form with both high school administrators and the university will be important to the success of the students. Family members should seek relationships with high school guidance counselors and teachers who can provide assistance in exposure to STEM activities as well as assistance with the college process. Families should strive to be engaged within the university community, along side their child. As universities evaluate and plan programs within a calendar 
that is well suited for the AKY region and provide financial support to attend; families should be committed to attending programs such as move in day, family weekend, and parent organizations within the university.

As a student begins their transition, it's important for families to remember that transitional success is both academic and non-academic (Venezia \& Jaeger, 2013). Students are transitioning to an entirely new community, so their barriers are enhanced compared to other students and subpopulations. Families will need to have an open dialogue and understanding of all changes and that some barriers will not be focused on academics.

\section{Communities}

Rural communities and legislators need to work together and find ways to ensure that the educational preparation within K-12 schools is occurring in a manner that supports higher education preparation. Checking the boxes of offering specific courses is not enough for success within a higher education setting. Legislation should focus on successful implementation of STEM education within the K-12 schools, as well as, growing the STEM economic development within the AKY community, and college access with the AKY region. Legislators should examine the possibility of offering additional STEM majors (even if offered at other state universities) at rural universities. The AKY communities should create a positive outlook on college which will play a role in the student's mindset of college as an option. Community coordinators could enhance a college going culture and educational programming to members within the community on the positive aspects of their children going to college.

Providing K-12 teachers with training on STEM education has the potential 
to enhance the implementation of STEM within the classroom. This is important as the new Kentucky K-12 education standards include components of STEM. Schools need to look at how they can incorporate STEM extracurricular programs such as Project Lead the Way, robotics teams or computer coding programs. These programs allow students to continue exploring their interest within STEM.

Communities should focus on creating Yosso's (2005) social capital for students who are considering attending college. This type of capital could be achieved through community programs, which provide college visit tours, community scholarships to college, and community campaigns highlighting successful college graduates as well as the benefits of going to college. Community leaders and members must understand the vital role they play within the success of educational attainment within the AKY region.

\section{Conclusion}

This study shared the lived experiences of students from AKY studying STEM at an urban university. Overall participants described their transition as stressful and painful (eight out of fourteen), while six participants claimed their transitions was easy. Through the analysis sub categories and themes were developed within the process of moving in, moving through, and moving out of the transition as well as pre-established themes, and the 4S's (self, support, situation and strategies). Analysis revealed that these participants needed a great deal of support throughout their transition. This support includes their family back at home and the community within college. Academic preparation was also stated as a major influencer within the transition; all participants mentioned this factor as either a strategy to transition or a stressor based on their perceived academic 
preparation from high school.

Examining a large subpopulation's transitional experience into college while studying a rigorous discipline such as STEM at an urban institution fills a gap in literature while also assisting various stakeholders in meeting their goals and objectives. AKY students could gain valuable coping mechanisms and realistic expectations. Through this research, much can be gained surrounding the experience of AKY students as well as potential interventions to enhance the transitional experience of this sub population. Ultimately the study provides a deeper understanding of transition and that the transition doesn't begin on day one of moving on to campus. Institutions should prioritize the facilitation of improving transitional experiences for students prior to their arrival on campus. The AKY community should rally behind and cheer for students who choose to attend an urban institution and study STEM, as opposed to disowning their community membership. Universities can gain awareness on practices and interventions, which can be implemented to support AKY students as they transition into college. Policy makers could benefit from this research by understanding the genuine experience of students who are transitioning into college and where to focus funding as well as support. Additionally, utilizing qualitative research provides a voice to the AKY students who are experiencing this transition within the literature which will advance the body of knowledge within college development and Schlossberg's transitional theory.

This research contributes to the literature in multiple ways: identification of the imbalance within the 4S system, need for partnership between families and universities, universities responsibility in supporting the student, family, and 
community, and the need for expansion of Schlossberg's transitional theory as it refers to attending college.

This research, more than diagrams or sub headings, gives me hope for the future. These findings provide possibilities for a region which I hold so dear in my heart. If our state, our communities, and our people come together then we have the potential to make meaningful change. If our universities can work to enhance educational attainment within the AKY region, if our legislators can increase STEM economic development within AKY, if our AKY communities can support the potential of higher educational and economic growth; only then we can begin to see true positive change within this oppressed region within Kentucky. 


\section{REFERENCES}

Anderson, M. L., Goodman, J., \& Schlossberg, N.K. (2012). Counseling adults in transition: Linking Schlossberg's theory with practice in a diverse world, 4th ed. New York: Springer Publishing Company, LLC.

Aspinwall, L. G., \& Taylor, S. E. (1992). Modeling cognitive adaptation: A longitudinal investigation of the impact of individual differences and coping on college adjustment and performance. Journal of Personality and Social Psychology, 63(6), 989-1003. https://doi.org/10.1037/0022-3514.63.6.989

Berger, J. B., \& Lyon, S. C. (2005). Past to present: A historical look at retention. In A. Seidman (Ed.), College student retention: Formula for student success (pp. 1-30). Westport, CT: Praeger Publishers.

Bryan, E., \& Simmons, L. (2009). Family involvement: Impacts on post-secondary educational success for first-generation Appalachian college students. Journal of College students Development, 50(4), 391-406

Bouteyre, E., Maurel, M, Bernaud, J. (2007). Daily hassles and depressive symptoms among first year psychology students in France: the role of coping and social support. Stress \& Health, 23(2), 93-99.

Camara, W (2013). Defining and measuring college and career readiness: A validation framework. Educational Measurement: Issues and Practice, 32(4), 16-27.

Chapman, A. (2017). Using the assessment process to overcome Imposter Syndrome in mature students. Journal of Further and Higher Education, 41(2), 112-119. 
Chickering, A. W., \& Schlossberg, N. K. (1995). Getting the most out of college. Needham Heights, MA: Allyn and Bacon.

Clark, M. R. (2005). Negotiating the freshmen year: Challenges and strategies among first-year college students. Journal of College Student Development, 46(3), 296316.

Collier, P., \& Morgan, D. (2008). "Is That Paper Really Due Today?": Differences in First-Generation and Traditional College Students' Understandings of Faculty Expectations. Higher Education, 55(4), 425-446. Retrieved June 16, 2020, from www.jstor.org/stable/29735194

Compas, B. E., Wagner, B. M., Slavin, L. A., \& Vannatta, K. (1986). A prospective study of life events, social support, and psychological symptomatology during the transition from high school to college. American Journal of Community Psychology, 14(3), 241-257. doi:10.1007/bf00911173

Conley, C. S., Kirsch, A. C., Dickson, D.A., \& Bryant, F.B. (2014). Negotiating the transition to college: Developmental trajectories and gender differences in psychological functioning, cognitive-affective strategies, and social well-being. Study of Emerging Adulthood, 2(3), 195-210.

County Economic Status, Fiscal Year 2018 (Rep.). (n.d.). Retrieved February 17, 2019, from Appalachian Regional Commission website: https://www.arc.gov/reports/custom_report.asp?REPORT_ID=71

Creswell, J.W., \& Poth, C.N (2018). Qualitative inquiry \& research design: Choosing among five approaches (4th ed.). Thousand Oaks, CA: Sage.

Davis-Kean, P. (2005). The influence of parent education and family income on child achievement: The indirect role of parental expectations and the home 
environment. Journal of Family Psychology: Journal of the Division of Family Psychology of the American Psychological Association (division 43),19(2), 294304.

Diekman, A. B., Brown, E. R., Johnston, A. M., \& Clark, E. K. (2010). Seeking congruity between goals and roles: A new look at why women opt out of Science, Technology, Engineering, and Mathematics careers. Psychological Science (09567976), 21(8), 1051-1057. https://doiorg.echo.louisville.edu/10.1177/0956797610377342

Dyk, P., \& Wilson, S. (1999). Family-based social capital considerations as predictors of attainments among Appalachian youth. Sociological Inquiry, 69(3), 477-503.

Edington, E., \& Conrad, J. (1981). Rural education: Issues in diversity. Educational Considerations, 8(3). doi:10.4148/0146-9282.1901

Engberg, M., \& Wolniak, G. C. (2013). College student pathways to the STEM disciplines. Teachers College Record, 115(1). Retrieved from Loyola eCommons, School of Education: Faculty Publication and other works.

Evans, N. (2010). Student development in college: Theory, research, and practice (2nd ed., Jossey-bass higher and adult education series). San Francisco: Jossey-Bass.

Folkman, S. \& Moskowitz, J. T. (2000). Positive affect and the other side of coping. American Psychologist, 55(6) 647-654.

Forum, B.-H. E. (2010). Increasing the Number of STEM Graduates : Insights from the U . S . STEM Education \& Modeling Project, 15.

Friedlander, L. J., Reid, G. J., Shupak, N., \& Cribbie, R. (2007). Social support, selfesteem, and stress as predictors of adjustment to university among first-year 
undergraduates. Journal of College Student Development, 48(3), 259-274. doi:10.1353/csd.2007.0024

Gale, T. \& Parker, S. (2014). Navigating change: A typology of student transition in higher education. Studies in Higher Education. 39, 10. 1080/03075079.2012.721351.

Gall, T. L, Evans, D. R., \& Bellerose, S (2000). Transition to first-year university: Patterns of change in adjustment across life domains and time. Journal of Social and Clinical Psychology, 19(4), 544-567.

Ganss, K. M. (2016). The college transition for first-year students from rural Oregon communities. Journal of Student Affairs Research and Practice, 53(3), 269-280. doi:10.1080/19496591.2016.1157487

Gardner, J. N., \& Siegel, M. J. (2001). Focusing on the first-year student. Priorities, 17, $1-17$.

Gibbs, R. M. (1989). College completion and return migration among rural youth. In R. M. Gibbs, PlL. Swaim, \& R. Teixeira (Eds.) Rural education and training in the new economy (pp. 61-80). Ames, Iowa: Iowa State University Press.

Holmstrom, L. L, Karp, D. A., \& Gray, P.S. (2012). Why Laundry, Not Hegel? Social class, transition to college, and pathways to adulthood. Symbolic Interactions, 25(4) 437- 462.

Huberman, M. B., Miles, A. M, \& Saldana, J (2014). Qualitative Data Analysis (3rd ed.). Thousand Oaks, CA: Sage.

Hudacs, A. (2017) An examination of college persistence factors for students from different rural communities: A multilevel analysis. Graduate College Dissertations and Theses. 682. http://scholarworks.uvm.edu/graddis/682 
Hultberg, J., K,. Plos, G.D., Hendry, \& K.I. Kjellgren. (2009). Scaffolding students’ transition to higher education: Parallel introductory courses for students and teachers. Journal of Further and Higher Education 32 (1), 47-57.

Increasing the number of STEM graduates: Insights from the U.S. STEM Education \& Modeling Project. (2010). Retrieved September, 2018, from http://www.bhef.com/sites/default/files/BHEF_2010_increasing_the_number_of_ stem_grads.pdf

Johnson, D. R. (2012). Campus racial climate perceptions and overall sense of belonging among racially diverse women in STEM majors. Journal of College Student Development, 53(2), 336-346.

Kelly, J.T., Kendrick, M.M., Newgent, R.A., \& Lucas, C.J. (2007). Strategies for student transition to college: a proactive approach, College Student Journal, 41(4), 10211035.

Komives, S. R., \& Brown, S. C. (n.d.). A facilitator guide for seniors: Four years in retrospect. Retrieved on 4-28-19, from http://www.newsreel.org/guides/seniorsg.htm

Lazarowicz, T. A. (2015). Understanding the transition experience of community college transfer students to a 4-year university: Incorporating Schlossberg’s transition theory into higher education p.207. Educational Administration: Theses, Dissertations, and Student Research. 216.

Leese, M (2010). Bridging the gap: Supporting student transitions into higher education. Journal of Further and Higher Education, 34(20), 239-251.

Leibowitz, Z. B., Schlossberg, N. K., \& Shore, J. E. (1991). Stopping the revolving door. Training \& Development Journal, 45(2), 43. 
Levitz, R. N. (2017). 2017 Recruitment Marketing Effective Practices. Retrieved September 15, 2019, from http://learn.ruffalonl.com/rs/395-EOG977/images/2017RecruitmentMarketingEffectivePracticesReport.pdf.

Li, Q., \& Swaminathan, H., \& Jiong Tang, (2009). Development of a classification system for Engineering student characteristics affecting college enrollment and retention. Journal of Engineering Education. 98. 10.1002/j.21689830.2009.tb01033.x.

Maples, M.R. (2000). Rural students' satisfaction with college environment: An ecological consideration. (Doctoral dissertation, The University of Iowa). Dissertation Abstracts International, 61(12), 4681. (UMI No. 9996133)

Martinez, J. A., Sher, K. J., Krull, J. L., \& Wood, P. K. (2009). Blue-collar scholars?: Mediators and moderators of university attrition in first-generation college students. Journal of College Student Development, 50(1), 87103. https://doi.org/10.1353/csd.0.0053

Mattanah, J. F., Ayers, J. F., Brand, B. L., Brooks, L. J., Quimby, J. L., \& Mcnary, S. W. (2010). A social support intervention to ease the college transition: Exploring main effects and moderators. Journal of College Student Development, 51(1), 93108. doi:10.1353/csd.0.0116

McCollough, C. A. (2011). Creating a college-going culture. Science Teacher, 78(3), 5155.

McCoy, D. L. (2004). A phenomenological approach to understanding first-generation college students' of color transitions to one “extreme” predominantly white institution. College Student Affairs Journal, 32(1), 155-169. 
McGhie, V. (2017). Entering university studies: Identifying enabling factors for transition from school to university. Higher Education, 73: 407 - 422. http://dx.doi.org/10.1007/s10734-016-0100-2

McMillan, W. (2013). Transition to university: The role played by emotion. European Journal of Dental Education, 17, 169-176.

Morris, E. A., Brooks, P. R., \& May, J.L. (2003). The relationship between achievement goal orientation and coping style: Traditional vs. nontraditional college students. College Student Journal, 37(1), 3-12.

Mulvenon, S. W. \& Robinson, D. H. (2014). The paradox of increasing both enrollment and graduation rate: Acknowledging elephants in the ivory tower. International Journal of Higher Education, 3(1), 66-70.

Nelson, C. A., 3rd, Zeanah, C. H., Fox, N. A., Marshall, P. J., Smyke, A. T., \& Guthrie, D. (2007). Cognitive recovery in socially deprived young children: the Bucharest Early Intervention Project. Science (New York, N.Y.), 318(5858), 1937-1940. https://doi.org/10.1126/science.1143921

National Center for Education Statistics (2003). Number of public school students’ by 2003 rural-urban continuum. Retrieved October 20, 2018, from http://nces.ed.gov/survey/RuralEd/TablesHTML/1beadledistricts.asp

National Student Clearinghouse research center (2018). Snapshot report, first-year persistence and retention. Retrieved March 3, 2019 from https://nscresearchcenter.org/wp-content/uploads/SnapshotReport33.pdf

Niu, L. (2016). Disparities in American graduate students’ tendency to borrow: Race, family background, and major. International Journal of Higher Education, 5(4). doi:10.5430/ijhe.v5n4p194 
Parenting practices and interventions among marginalized families in Appalachia:

Building on family strengths. (1996). Family Relations, 45(4), 387-387. doi:10.2307/585168

Perna, L. W. (n.d.). Studying college access and choice: A proposed conceptual model. Higher Education: Handbook of Theory and Research, 99-157. doi:10.1007/14020-4512-3_3

Pike, G. \& Kuh, G. (2005). First and Second-Generation College Students: A Comparison of Their Engagement and Intellectual Development. The Journal of Higher Education. 76. 276-300. 10.1353/jhe.2005.0021.

Pollard, K., \& Jacobsen, L. (2011, September). Appalachian Region Commission. In The Appalachian region in 2010: A census data overview chartbook. Retrieved October, 2018, from https://assets.prb.org/pdf12/appalachia-census-chartbook2011.pdf

Postareff, L., Mattsson, M., Lindblom-Ylanne, S., \& Hailikari, T. (2017, March). The complex relationship between emotions, approaches to learning, study success and study progress during the transition to university. Higher Education, 73(3), 441-457.

Poverty Rates 2012-2016 (Rep.). (n.d.). Retrieved February 17, 2019, from Appalachian Regional Commission website: https://www.arc.gov/reports/custom_report.asp?REPORT_ID=72

Powers, M. S. (2010). Applying Schlossberg's Transition Theory to Nontraditional Male Drop-outs. Retrieved from ProQuest Dissertations and Theses (UMI Number: 3397864). 
Pulcini, B. (2016). Appreciative advising to promote degree completion by Appalachian women. NACADA Journal, 36(2), 47-53

Reid, M. \& III, James. (2008). College Readiness and Academic Preparation for Postsecondary Education: Oral Histories of First-Generation Urban College Students. Urban Education - URBAN EDUC. 43. 240-261. 10.1177/0042085907312346.

Rendon, L. I. (1995, March). Facilitating retention and transfer for first-generation students in community colleges. Paper presented at the New Mexico Institute: Rural Community College Initiative, Espanola, NM. (ED 383 369)

Ryan, S. W., Carlstrom, A. H., Hughey, \& Harris, B. S. (2011). From boots to books: Applying Schlossberg's model to transitioning American veterans. NACADA Journal: Spring, 31(1), 55-63.

Schaffhauser, D. (2018, January 08). STEM Majors on Rise even as College Enrollment Shrinks. Retrieved March 4, 2019, from https://campustechnology.com/articles/2018/01/10/stem-majors-on-rise-even-ascollege-enrollment-shrinks.aspx

Schlossberg, N. K. (1981). A model for analyzing human adaptation to transition. The Counseling Psychologist, 9(2), 2-18. doi:10.1177/001100008100900202

Schlossberg, N.K. (1984). Counseling adults in transition. New York: Springer.

Schwartz, S. J., Côté, J. E., \& Arnett, J. J. (2005). Identity and agency in emerging adulthood: Two developmental routes in the individualization process. Youth \& Society, 37(2), 201-229. https://doi.org/10.1177/0044118X05275965

Seltzer, R. (2017, September). Disparaging interpretive dance (and More). Inside Higher Ed. Retrieved October, 2018, from 
https://www.insidehighered.com/news/2017/09/14/kentuckys-governor-saysuniversities-should-think-about-cutting-programs-poor-job

Shute, Valerie \& Hansen, Eric \& Underwood, Jody \& Razzouk, Rim. (2011). A Review of the Relationship between Parental Involvement and Secondary School Students' Academic Achievement. Education Research International. 10. 10.1155/2011/915326.

Simmon, R. A., Aulls, M.W., Dedic, H, Hubbard, K, \& Hall, N.C. (2015). Exploring student persistence in STEM Programs: A motivational model. Canadian Journal of Education 38(1), 1-27.

Smith, B. F., \& Brackin, R. (1993). Components of a comprehensive orientation program. In M. L. Upcraft (Ed.), Designing successful transitions: A guide for orienting students to college (Monograph No. 13, pp. 35-48). Columbia, SC: National Resource Center for the Freshman Year Experience, University of South Carolina.

Stone, C. (2014) Rural Routes: First year college experiences of students from rural backgrounds. Graduate College Dissertations and Thesis. 3385. https://scholarworks.montana.edu/xmlui/handle/1/3385

St. John, E.P., Paulsen, M.B. \& Starkey, J.B. The nexus between college choice and persistence. Res High Educ 37, 175-220 (1996). https://doi.org/10.1007/BF01730115

Tinto, V. (1993). Leaving college: Rethinking the causes and cures of student attrition (2nd ed.). Chicago: The University of Chicago Press

Upcraft, M. L., \& Gardner, J. N. (1989). A comprehensive approach to enhancing freshman success. In M. L. Upcraft \& J. N. Gardner (Eds.), The freshman year 
experience: Helping students survive and succeed in college (pp. 1-12). San Francisco: Jossey-Bass.

Voelker R (2003). Mounting student depression taxing campus mental health services. JAMA, 289: 2055-2056.

Wang, T. R. (2014). Formational turning points in the transition to college: Understanding how communication events shape first-generation students' pedagogical and interpersonal relationships. Communication Education, 63(1), 63-82.

Wheeler, H. (2012). Veterans' transitions to community college: A case study. Community College Journal of Research and Practice, 36(10), 775-792.

William, L. (2011). From high school to college: The transition experiences of black and white students. Journal of Black Studies, 42(5), 828-845.

Wolcott, G. V. (2006). Critical Reflections of College Sophomores About Preparation and Persistence During Their Freshman Year. Journal of College Orientation, Transition, and Retention, 14(1). https://doi.org/10.24926/jcotr.v14i1.2652

Wong, B.T. (2006). 2006 College Academic All-Stars First Team. Retrieved January 14, 2019 from http://www.usatoday.com/news/education/2005-02-16-collegeallstarsfirst-team_x.htm

Workman, J. (2015). Parental influence on exploratory students’ college choice, major, and career decision making. College Student Journal, 49(1), 23-30.

Venezia, A., \& Jaeger, L. (2013). Transitions from high school to college. The future of children, 23(1), 117-136. doi:10.1353/foc.2013.0004 
Xu, Y. J. (2015). Attention to retention: Exploring and addressing the needs of college students in STEM majors. Journal of Education and Training Studies, 4(2), 6776. http://doi.org/10.11114/jets.v4i2.114

Yoder, B. L. (2012). Going the distance: Best practices and strategies for retaining engineering, engineering technology and computing students. Computer. American Society of Engineering Education. Retrieved October 20, 2017, from https://www.asee.org/retention-project

Yosso, T.J (2005). Whose culture has capital? A critical race theory discussion of community. 


\section{APPENDIX A: Recruitment Email}

\section{Subject: Appalachian student experience research}

Date

Dear (Participant Name),

I am a student enrolled at the University of Louisville where I am pursing my doctoral degree. I am writing to ask for your help. Would you be interested in participating in a study of Appalachian KY students who are enrolled in a STEM major? The purpose of this study is to examine how rural Appalachian students attending an urban large university and majoring in STEM described their perception of their situation, self, support and strategies throughout their transition. This study is being conducted for educational purposes and could be used by the college to determine how to better address the needs of rural students studying STEM at UofL. This study is under the supervision of Dr. Alagaraja, 502-852-0617 meera.alagaraja@louisville.edu and assisted by Heidi Neal 502-852-0364 heidi.neal@louisville.edu

We will be offered two options to participate: a focus group (6-12 individuals) or a oneon-one interview. The study group will be hosted Date, Time, Location and date, time, location. One-on-one interviews will occur between date and date. Please RSVP to Heidi Neal, Heidi.neal@louisville.edu

Your answers in the study will be confidential and participation is voluntary. Your help would be greatly appreciated in furthering our understanding of your experience. If you have any questions, please don’t hesitate to let me know.

Sincerely,

Meera Alagaraja, $\mathrm{PhD}$

Principal Investigator

502-852-0617

College of Education, Rm 347

Heidi Neal

PhD student, College of Education 


\section{APPENDIX B: Interview Protocol}

\section{Transition Interview}

Interview Overview:

Name:

Date,Time, and Location:

Interview Purpose:

1. To establish trust with the participant

2. To understand their experience of transitioning into college at a urban institution

3. To understand the factors which may have presented additional challenges because as they

moved in and out of the transition

Interview Procedure:

Before the interview: Remind the participant that the interview will be audio recorded.

Ensure there is enough time to allow interview to go as long as they would . Determine a location that is quiet and wont' receive distraction (if in office, make sure the blinds are drawn).

1. Welcome interviewee.

2. Introduce yourself and the research study

3. Provide overview of study

a. The estimated time commitment.

b. Ensure that the conversation will be confidential.

c. Tape recording and note taking but individual’s identity remains confidential 
d. Interviewees receive a copy of the transcribed interview for review and feedback/ edit

4. Answer any questions

5. Begin interview.

6. Stop recorder at the end of interview.

7. Thank interviewee.

Potential Questions:

1. Introduction/ description of study

2. Where did you grow up?

Situation:

1. Talk about your experience growing up.

2. Describe your high school involvement/ academic development

a. How did you get involved in these activities?

3. What made you decide to study STEM in college?

4. What made you decide to attend UofL?

a. Did you receive any assistance or guidance in your college selection?

b. Who did you have conversations about in selecting your University?

Parents/Counselors/Friends/Teachers/Coaches

5. How did your academic

Strategies:

6. What did you look forward to as you prepared to transition to UofL?

7. What did you worry about as you prepared to transition to UofL?

8. Who has been significant in your transition to UofL? What events have been significant? 


\section{Support Systems:}

1. What groups/ organizations have you joined here at UofL?

2. What services do exist at UofL to assist with your transition?

3. Who do/ would you go to with academic support?

4. What is the most important form of support you need during your transition?

5. What role does your family play in your transition?

Self:

1. Reflecting on your transition to UofL, what do you wish you had done differently?

2. Do you think being from rural KY has any advantages/ disadvantages coming to UofL and studying STEM?

3. How do you think being a rural KY student will affect your career path?

a. Do you plan to return to Eastern KY?

Other:

1. What advice do you have for other rural KY students studying STEM?

2. Is there anything I should have asked you that I didn't? 


\section{APPENDIX C: Focus Group Protocol}

\section{Transition Focus Group}

\section{Focus Group Overview:}

Name:

Date,Time, and Location:

\section{Interview Purpose:}

1. To establish trust with the participant

2. To understand the experience and resources utilized during the transitioning into college at an urban institution

3. To understand the factors which may have presented additional challenges because as they moved in and out of the transition Interview Procedure:

Before the focus group: Before the focus group: Remind participants that the focus group will be audio recorded. Ensure there is enough time to allow focus groups to go as long as they would . Determine a location that is quiet and won't receive distraction (if in office, make sure the blinds are drawn).

1. Welcome interviewee.

2. Introduce yourself and the research study

3. Provide overview of study

a. The estimated time commitment.

b. Ensure that the conversation will be confidential.

c. Tape recording and note taking but individuals identity remain confidential

4. Answer any questions

5. Begin interview.

6. Stop recorder at the end of interview.

7. Thank interviewee.

\section{Potential Questions:}


1. Introduction/ description of study

2. Where did you grow up?

\section{Situation:}

1. What made you decide to attend UofL?

a. Did you receive any assistance or guidance in your college selection?

b. Who did you have conversations about in selecting your University? Parents/Counselors/Friends/Teachers/Coaches

\section{Support Systems:}

1. What groups/ organizations have you joined here at UofL?

2. What services do exist at UofL to assist with your transition?

3. Who do/ would you go to for academic support?

4. What is the most important form of support you need during your transition?

5. What role does your family play in your transition?

\section{Other:}

1. What advice do you have for other rural KY students studying STEM?

2. Is there anything I should have asked you that I didn't? 
APPENDIX D: First cycle coding

\begin{tabular}{|c|c|c|c|}
\hline Name & Description & Files & References \\
\hline Academic Prep & & 3 & 9 \\
\hline ACT test taking & & 0 & 0 \\
\hline adaptation & & 3 & 10 \\
\hline changes to self & & 2 & 4 \\
\hline driving & & 2 & 2 \\
\hline Poorly prepared & & 2 & 3 \\
\hline $\begin{array}{l}\text { Summer prep in } \\
\text { programs }\end{array}$ & & 1 & 1 \\
\hline big city & & 3 & 4 \\
\hline $\begin{array}{l}\text { neg. response from } \\
\text { community }\end{array}$ & & 1 & 1 \\
\hline scary town & & 2 & 3 \\
\hline college going culture & & 2 & 8 \\
\hline ACT test & & 1 & 1 \\
\hline dual credit & & 1 & 1 \\
\hline $\begin{array}{l}\text { guidance } \\
\text { counsellors }\end{array}$ & & 1 & 1 \\
\hline parents & & 1 & 1 \\
\hline
\end{tabular}




\begin{tabular}{|c|c|c|c|}
\hline Name & Description & Files & References \\
\hline teacher & & 1 & 2 \\
\hline education symbol & & 3 & 5 \\
\hline Better Job & & 0 & 0 \\
\hline goofy movie & & 1 & 1 \\
\hline key & & 1 & 1 \\
\hline Opportunities & & 1 & 2 \\
\hline personal growth & & 1 & 1 \\
\hline home town & & 4 & 17 \\
\hline $\begin{array}{l}\text { everyone knows } \\
\text { everyone }\end{array}$ & & 1 & 1 \\
\hline excited to leave & & 1 & 1 \\
\hline $\begin{array}{l}\text { friendly } \\
\text { community }\end{array}$ & & 1 & 1 \\
\hline negative emotion & & 2 & 5 \\
\hline out of place & & 1 & 2 \\
\hline sad to leave & & 1 & 1 \\
\hline Idea & & 0 & 0 \\
\hline Move back home & & 3 & 3 \\
\hline Self & demo char., individual prep & 2 & 6 \\
\hline
\end{tabular}




\begin{tabular}{|c|c|c|c|}
\hline Name & Description & Files & References \\
\hline Situation & $\begin{array}{l}\text { Timing of transition, planned or } \\
\text { unplanned, expectations }\end{array}$ & 0 & 0 \\
\hline Slap in the face & & 3 & 3 \\
\hline Strategies & Coping methods throughout the transition & 4 & 8 \\
\hline Support & $\begin{array}{l}\text { Individuals support moving in, during } \\
\text { and out of the transition }\end{array}$ & 4 & 17 \\
\hline Advising & & 1 & 2 \\
\hline Family & & 1 & 5 \\
\hline friends & & 1 & 3 \\
\hline Individual & & 1 & 1 \\
\hline $\begin{array}{l}\text { major student } \\
\text { group }\end{array}$ & & 1 & 1 \\
\hline on campus jobs & & 1 & 1 \\
\hline Religious & & 0 & 0 \\
\hline Sorority & & 0 & 0 \\
\hline sports team & & 1 & 1 \\
\hline Why attend UofL & & 3 & 6 \\
\hline why study STEM & & 2 & 2 \\
\hline
\end{tabular}




\section{CURRICULUM VITAE}

\section{Heidi A. Neal, Ph.D}

\section{Profile}

Accomplished project manager, supervisory experience, strong communication skills, grant writer, professor, and philanthropist. Proficient in Microsoft Outlook, Oracle Banner, PeopleSoft, JobZone, Blackboard Analytics. Record of success in developing marketing campaigns, recruitment strategies, strategic planning, retention plans and assessment/evaluation. Recognized for ability to build relationships with key stakeholders. Exceptional trainer and mentor with skills to motivate individual performance from team members.

\section{Work Experience}

\section{Director of Student Success \& Student Affairs - JB Speed School of Engineering, University of Louisville 2014-present}

Interim Assistant Dean of Student Affairs- JB Speed School of Engineering, University of Louisville 2017-2018

- Provide overall leadership of Engineering student experience, advising, career development, student engagement, outreach

- $\quad$ Secure \$250,000 in funding for student success and outreach initiatives annually

- Oversight of $\$ 2,000,000+$ yearly budget

- $\quad$ Provide overall leadership and direction in support of student success, retention and graduation rates 95\% re-enrollment rate for freshmen engineering course

6\% increase in 1 to 2 year and 2 to 3-year retention fall '16-'17, fall '17-18

- $\quad$ Oversee 10 full time staff, 3 graduate interns and 12 student workers

- Lead the direction of the student experience from admission until graduation Developed tracking and predictive model for engineering students Implemented early alert notification interventions from faculty for at risk students

- Serve as point of contact for students and faculty/staff regarding retention risk factors; identify atrisk students and assure individualized outreach to develop individual plans for success

- Handle student complaints, grievances, counseling needs and cases of student misconduct

- $\quad$ Employ current retention management practices and strategies to develop and implement a comprehensive student success plan Developed and implementing 2-year strategic plan Oversee development and implementation of Speed mentoring

Project manager for the implementation of Symplicity Insight Advising software (moved from paper file to complete online student success file), 4-month training program and 2 year UofL pilot program

Collaborate with Faculty and staff on course needs, classroom limits and forecasting of course offerings

Provide substitution review for courses, course load limit and academic affairs decisions

- $\quad$ Develop appropriate assessment and analytics to support student success initiatives

- Cooperate with university partners in carrying out university-wide advising and retention initiatives, including Flight Plan 
- Act as liaison with faculty, academic advisors/counselors and support staff to promote student activities and improvement of student life; coordinate student special events (commencement, honors \& awards, etc.)

- Develop outreach programs to high school students to prepare students for college success and provide support for K-8 programs

Participated in University pilot program for minors on campus safety

Oversight and tracking of annual summer camps

Created a University Signature Partnership with the West End School, Nativity Academy and

Central High School to include: maker space, scholarships, mentoring and quarterly hack a thons

Developed and opened 3 Speed School maker spaces

Increased outreach from 2,500 to over 7,000 K-12 students (doubling outreach programming in 1 year)

Created dual enrollment programs with top district schools

- Established "Education Partnership" between Speed School and VEX Robotics World championship

Created \$60,000 in VEX scholarships, campus tours, 200 hours of Speed School volunteers and received: announcement e-blast, logo link from all web outreach, special event invites, and UofL branding throughout the championship reaching over 20,000 potential students and 30 countries

- Lead and support the diversity initiatives of the School

Established a Speed School LGBT and ally student organization

Opened the first Speed School gender natural bathroom \& safe space for LGBT community

Created a Speed School campus climate plan

Remodeled minority student organization space

Secured $\$ 60,000$ for freshmen at risk bridge program

- Oversees Academic Services including: course enrollment management, course predictions and classroom management

Implement first year block scheduling and predictive enrollment course needs

- Secured funding and implemented the Brown Forman Bridge Program: At-risk residential program to build academic and student success transitioning skills

- Title IX certificate officer and collaborate with Dean of Students on situations within the engineering school

- Oversee summer orientation sessions

- Secured funds and opened the Academic \& Leadership Center (offering free tutoring and leadership development)

Free tutoring and leadership development programs

Increased first year tutoring services

- Oversight of the Engineering Living and Learning Community for $1^{\text {st }}$ year students

Expanded the community from 100 students to 217 freshmen

Developed intentional educational and social programming focused on student's interest

Developed and implemented research course for ELLC students

ELLC cohort continuously has higher retention and GPA over engineering cohort

- Work closely with UG affairs, Registrar, Bursar, Financial Aid, Dean of Students, Risk

Management, IR

MBA \& Graduate Programs Recruiting Coordinator - University of Louisville March 2013-June 2014

- Developed strategic recruitment planning for local, region, and national recruiting

- Recruit high profile candidates

- Research, develop, and coordinate sponsorship opportunities for the College of Business ranging from $\$ 200$ - \$5,000 Kentucky Women’s leadership conference, Kentucky state chamber of commerce, Southern Indiana Chamber of Commerce, Women’s MBA non-profit, Louisville Venture Connectors, etc

- Attend over 80 community events on behalf of the MBA office yearly

- Manage communication and application process for 400+ applicants

Developed prospect and applicant community plan 
- $\quad$ Collect \& Analyze data on MBA application trends and event assessment

- Assist in managing class registration, degree completion plans, probation and graduate degree checks for over 250 students Improved process and procures of cohort checks through blackboard analytics

- Manage over 20 MBA events a year including: information sessions, recruitment events, leadership luncheon, alumni events, and graduation

Increased outreach recruitment events from 2 to 25 a year

Implemented student recruitment and student engagement opportunities

- Oversee the development of the MBA Alumni Association

Developed 6 month strategic plan

Coordinated developed of Board of Advisors, executive council and committee chairs

Hosted first Alumni Network event, Alumni training and board meeting

Associate Director for Enrollment Services \& Career Services - Ivy Tech Community College 2011 2013

- Directly oversee the Admissions Office \& Career Services

Supervising between 5-7 staff members

Oversee office schedule, territory distribution, time off requests, evaluations, professional development

- Manage daily operations of offices

Develop \& present enrollment reports, Data input, application processing, document imaging, potential student/ student meeting, individualize academic success plans, individual student meetings for enrollment, career planning, resume critics, mock interviewing

- Develop, execute, and assess application to enrollment strategies

Evaluate and explain application numbers, enrollment number, strategies, and design recruitment plan for campus community

- Increased high school visit programs by $8 \%$, community outreach programs by $12 \%$ and on-campus visits by $23 \%$

- Execute large scale community and college wide events

Fall, Summer, and Spring Open House

Developed $1^{\text {st }}$ ever Career Week: Etiquette Dinner, Networking Night, and Job Fair

Average over 150 attendants at each event

- Built relationships with over 100 different community companies, non profits, and schools

- Developed centralized internship program for campus community

Develop local internship database and marketing efforts

Brought all current and new internship sites up to NACE and CDPI standards

Create, Track, and Evaluate internships \& internship sites

- Developed new marketing and branding efforts for Career Services including: student outreach, employer outreach, faculty and staff outreach, employment marketing, and options to assist employers hire Ivy Tech graduates

- Adjunct Sociology Professor: Introduction to Sociology, Multicultural Affairs and Social Problems

\section{Assistant Director for Student Leadership and Civic Engagement - Nova Southeastern University} $2009-2011$

- Directly advised the Leadership and Civic Engagement development of our undergraduate population

- Assisted in the implementation of a $\$ 300,000$ annual leadership program for 25 incoming freshmen

- Managed a \$35,000 service operating budget and \$300,000 scholarship operating budget

- Served as the direct leader of office recruitment and retention

Developed avenues to increase involvement of undergraduate students, faculty, staff, and alumni Increased involvement of program attendance and retention by $85 \%$ in $2009-2010$ 
Oversaw all evaluation and assessment of individual program and office success

Directly advised office communication plan to ensure students are on a successful track to stay in office programs

- Executed special large-scale conferences, upscale receptions, weekend retreats, weeklong service trips

Annual Leadership Conference with an average 175 attendants

Create and implement 7 service trips with 150 attendees

Event planning for donor receptions and symposium for scholarship award recipients

Plan and execute the annual University Leadership Awards Banquet with an average of 150

attendants

- $\quad$ Developed and Implemented Service Study Trips (local, national, and international)

Increased service trips from 1 in 2009 to 7 in 2010

Developed all marketing and recruitment tactics

Wrote week curriculum for trip

Evaluated and Assess success of each trip through quantitative and qualitative research

Solicited Service Trip Sponsorships from \$350 - \$2,500

\section{Education}

University of Louisville- Louisville, KY (2016-2020)

Ph.D- Educational Leadership and Organizational Development: Concentration in Higher Education Administration

East Tennessee State University - Johnson City, TN

Master of Arts - Sociology: Concentration in Applied Sociology

Morehead State University - Morehead, KY

Bachelor of Arts - Sociology: Emphasis in Regional Analysis and Public Policy (IRAPP)

Service to the University

UofL Strategic planning, Work/ Campus Climate group

Staff Senator- College of Engineering

Committee member

UofL community engagement committee member

UofL Commission on the Status of Women, committee member

\section{Professional Presentations and Experience}

ACPA National Conference Presenter 2017

Community in Schools, Board of Directors (2015-2016)

Leadership Southern Indiana, Board of Advisors (2014-2016)

LSI ENGAGE, Committee chair (2015-2017)

MSU Alumni Board of Directors (2014 - 2016)

KY Admissions Conference Presenter 2014, 2016

ACPA state conference Presenter 2015

Kentucky and Ohio Association Conference Presenter 2015

Honors/ Interests

2018 INSIGHT into Diversity, Inspiring STEM programs, Office of Student Success

2016 Leadership Southern Indiana, Volunteer of the Year

201620 under 40 Southern Indiana, Business Source

2015 Provost's Leadership Academy Member

2014 'Way to go Woman', Today's Woman magazine, leadership in education award for Louisville professors under 40

2014 Southern Indiana Chamber of Commerce Business Awards Judge

2013 Data analyzer for research topic "Ethics in Business during economic recession”

2011 Top 20 Universities dedicated to Civic Engagement: USA Today news and world report
UofL Strategic planning, Learn/ retention grou CPE \& University Wide Academic Review Speed School Diversity Committee UofL employee mentoring program 